\title{
GEODESICS IN PIECEWISE LINEAR MANIFOLDS
}

\author{
BY
}

\author{
DAVID A. STONE(1)
}

\begin{abstract}
A simplicial complex $M$ is metrized by assigning to each simplex $a \in M$ a linear simplex $a^{*}$ in some Euclidean space $\mathbf{R}^{k}$ so that face relations correspond to isometries. An equivalence class of metrized complexes under the relation generated by subdivisions and isometries is called a metric complex; it consists primarily of a polyhedron $M$ with an intrinsic metric $\rho_{M}$. This paper studies geodesics in metric complexes. Let $P \in M$; then the tangent space $T_{P}(\mathrm{M})$ is canonically isometric to an orthogonal product of cones from $P, \quad \mathrm{R}^{k} \times \nu_{P}(\mathrm{M})$; once $k$ is as large as possible. $\nu_{P}(\mathrm{M})$ is called the normal geometry at $P$ in $M$. Let $P \bar{X}$ be a tangent direction at $P$ in $\nu_{P}(M)$. I define numbers $\kappa_{+}(P \bar{X})$ and $\kappa_{-}(P \bar{X})$, called the maximum and minimum curvatures at $P$ in the direction $P \bar{X}$. THEOREM. Let $\mathrm{M}$ be a complete, simply-connected metric complex which is a p.l. n-manifold without boundary. Assume $\kappa_{+}(P \bar{X})$ $<0$ for all $P \in M$ and all $P \bar{X} \subseteq \nu_{P}(M)$. Then $M$ is p.l. isomorphic to $R^{n}$. This is analogous to a well-known theorem for smooth manifolds by $\mathrm{E}$. Cartan and J. Hadamard. THEOREM (RGUGHLY). Let $M$ be a complete metric complex which is a p.l. n-manifold without boundary. Assume (1) there is a number $\kappa \geqslant 0$ such that $\kappa \_(P \bar{X}) \geqslant \kappa$ whenever $P$ is in the $(n-2)$-skeleton of $M$ and whenever $P \bar{X} \subseteq \nu_{P}(M) ;(2)$ the simplexes of $M$ are bounded in size and shape. Then $M$ is compact. This is analogous to a weak form of a well-known theorem of S. B. Myers for smooth manifolds.
\end{abstract}

1. Introduction. I have been studying geodesics on manifolds with piecewise-linear (abbreviated to p.1.) metrics, trying to relate the global topology of such a manifold to its local geometry. In $\$ 2$ I shall lead up to the definition, for any point $P$ of a p.l. manifold $\mathrm{M}$, and for any tangent direction $P \bar{X}$ at $P$ which lies in the "normal geometry" $\nu_{P}(\mathbf{M})$ at $P$ in $\mathbf{M}$, of numbers $\kappa_{+}(P \bar{X})$ and $\kappa_{-}(P \bar{X})$, with $\kappa_{+}(P \bar{X}) \geqslant \kappa_{-}(P \bar{X})$. They are called the "maximum and minimum curvatures" of $M$ at $P$ in the direction $P \bar{X}$. There seems to be an analogy between $\kappa_{-}(P \bar{X})$ and, in the smooth case, the minimum sectional curvature at a point of two-planes containing a fixed tangent vector at that point; likewise between $\kappa_{+}(P \bar{X})$ and the maximum such sectional curvature. To support this intuition I offer the following results:

Received by the editors August 7, 1973 and, in revised form, November 8, 1974.

AMS (MOS) subject classifications (1970). Primary 53C20, 53C70, 57C99; Secondary $57 \mathrm{D} 70,53 \mathrm{C} 45$.

(1) I am glad to thank publicly some of those who helped me: T. Akiba, B. Sanderson and E. Stone; also the National Science Foundation for Grants P22927 and P029431000. 
THEOREM 1. Let $\mathrm{M}$ be a simply-connected, complete metric complex which is a p.l. n-manifold without boundary. If $\kappa_{+}(P \bar{X}) \leqslant 0$ for each $P \in \mathbf{M}$ and for each tangent direction $P \bar{X} \subseteq \nu_{P}(\mathbf{M})$, then $\mathbf{M}$ is p.l. isomorphic to Euclidean space $\mathbf{R}^{n}$.

(This theorem is slightly stronger than the corresponding one announced in [13] .) Theorem 1 is analogous to a theorem proved for smooth manifolds by E. Cartan [3] and J. Hadamard [5] under the hypothesis that every sectional curvature be $\leqslant 0$.

THEOREM 2. Let $\mathbf{M}$ be a complete metric complex which is an n-manifold without boundary. Assume that whenever $a$ is an $(n-2)$-simplex, $P \in$ int $a$ and $P \bar{X} \subseteq v_{P}(\mathrm{M})$, then $\kappa_{-}(P \bar{X}) \geqslant 0$. Then:

(i) $M$ has positive curvature "everywhere": $\kappa_{-}(P \bar{X}) \geqslant 0$ for all $P \bar{X} \subseteq \nu_{P}(M)$, provided that $P$ is in the $(n-2)$-skeleton of $\mathbf{M}$;

(ii) if $n$ is even and $\mathbf{M}$ orientable, then $\mathbf{M}$ is simply-connected;

(iii) if $n$ is odd, then $\mathbf{M}$ is orientable.

In the smooth case a theorem analogous to (ii), was proved by J. Synge [14] under the assumption that all sectional curvatures are $\geqslant 0$; the smooth analogue of (iii) is an elementary consequence of his method observed by A. Preissmann [9].

THEOREM 3. Let $\mathrm{M}$ be a complete metric complex which is an n-manifold without boundary. Assume

(1) there is a number $\kappa \geqslant 0$ such that whenever $a$ is an $(n-2)$-simplex, $P \in$ int $a$ and $P \bar{X} \subseteq \nu_{P}(M)$, then $\kappa_{-}(P \bar{X}) \geqslant \kappa$;

(2) there is a number $\mathbf{p}$ such that whenever $a$ is an n-simplex of $\mathbf{M}$ and is represented as a linear simplex in $R^{n}$, then the $(n-1)$-sphere circumscribed about $a$ has radius $\leqslant \mathrm{p}$.

Then

(i) M has positive curvature "everywhere", as in Theorem 2(i);

(ii) $\mathbf{M}$ is compact (I shall give an estimate for the diameter of $\mathbf{M}$ ).

Theorem 3 is a weak analogue of a theorem proved for smooth manifolds by S. Myers [7] under the hypothesis that the Ricci curvature be everywhere bounded above 0 . I have no idea what notion of pl. Ricci curvature would imply the "right" analogue of Myers' theorem, but I do believe that some such notion exists.

An amusing consequence of Theorem 3 is

THEOREM 4. Let $\mathbf{K}$ be a simplicial 3-manifold without boundary. Assume that every 1-simplex is a face of at most five 3-simplexes. Then $\mathbf{K}$ is finite. 
A. Phillips has pointed out that $\mathbf{R}^{3}$ can be triangulated so that every 1 -simplex is a face of at most six 3-simplexes. The proof of the theorem is to give $\mathbf{K}$ a metric by making all the tetrahedra regular, of side-length 1 , and then verifying that the hypotheses of Theorem 3 hold. I should be most interested to know of a combinatorial proof of Theorem 4; the more so because I have been able to prove by combinatorial means a general finiteness theorem for simplicial manifolds, $\left({ }^{2}\right)$ but Theorem 4 is a "limiting case" and I cannot prove it by these methods.

For 2-dimensional manifolds-topological and p..-the theory of curvature is well established (see Aleksandroff and Zalgaller [1] or W. Rinow [10]), and Theorems 1, 2 and 3, though perhaps new, are simply exercises. The present work is independent of both T. Banchoff's work [2] on the Gauss-Bonnet theorem for polyhedra and of H. Osborn's work [8] on deRham theory for p.l. manifolds. I have benefitted greatly from $\mathrm{H}$. Gluck's foundational work on the intrinsic geometry of polyhedra; much of $\S 2$ is based on his notes [4].

2. Foundations. First, some abbreviations, symbols and conventions.

In general, bold-face letters such as $\mathbf{X}, \mathbf{M}$ will denote both topological spaces and simplicial complexes. I shall make no distinction in notation between an abstract simplicial complex $\mathbf{M}$, a particular realization of $\mathbf{M}$ (such as a metric complex, to be defined) and the underlying topological space of a realization of $\mathbf{M}$. Lower-case letters $a, b$ will refer to simplexes, and $v, w$ to vertices, of a simplicial complex. Capitals such as $P, Q$ will refer to points in topological spaces. Thus in the statements " $a \in M$ ", " $P \in \mathbf{M}$ ", " $P \in a$ ", $a$ and $M$ are a simplex and a simplicial complex in the first statement, and topological spaces in the other two.

The dimension of a simplicial complex or simplex will be written $\operatorname{dim} \mathbf{M}$ or $\operatorname{dim} a$. The notation $b<a$ will mean that $b$ is a face of $a$. If $b_{1}, \ldots, b_{k}$ are faces of $a$, then their span $b_{1}, \ldots, b_{k}$ is the smallest face of $a$ which contains them all.

$\mathbf{R}$ will denote the real line, I the unit interval $[0,1] . \mathbf{R}^{n}$ will denote Euclidean $n$-space; thus $\mathbf{R}^{\mathbf{1}}$ is not quite the same as $\mathbf{R}$. By a sphere $S^{n}$, I shall mean the locus of a point in $\mathbf{R}^{n+1}$ at fixed distance from a fixed point. If $\mathbf{X} \subseteq \mathbf{R}^{n}$, then the affine space generated by $X$, denoted $[X]$, is defined to be the smallest affine space in $\mathbf{R}^{n}$ which contains $\mathrm{X}$. If $\mathrm{X}, \mathrm{Y} \subseteq \mathrm{R}^{n}$, then their join $\mathrm{X} * \mathrm{Y}$ is defined as $\bigcup\{$ straight-line segments from $X$ to $Y$, for $X \in \mathrm{X}, Y \in \mathrm{Y}\}$. If $\mathrm{X}, \mathrm{Y} \subseteq \mathrm{S}^{n}$ and if no point of $X$ is antipodal to any point of $Y$, then $X * Y$ is defined in the same way, but with "straight-line segments" replaced by "shortest geodesic segments".

If $\mathbf{X} \subseteq \mathbf{Y}$, then int $\mathbf{X}$, bdy $\mathbf{X}$ and $\operatorname{cl}[\mathbf{X}]$ will denote the interior, boundary and closure of $\mathbf{X}$ in $\mathbf{Y}$. If $\mathbf{X}$ is a manifold, then bdy $\mathbf{X}$ will refer to its boundary. The diameter of a metric space $\mathbf{X}$ will be written diam $\mathbf{X}$. If $f: \mathbf{X} \rightarrow \mathbf{R}$ and $r \in$ $\mathbf{R}$, then $f^{r}$ will denote $\{X \in X$ such that $f(X) \leqslant r\}$.

$\left({ }^{2}\right)$ Added in proof. I retract this statement. 
I shall abbreviate piecewise linear to p.l. and piecewise differentiable to p.d.

Let $\mathbf{M}$ be a connected, locally-finite, finite-dimensional simplicial complex. $\mathbf{M}$ is metrized by giving, for each $a \in \mathbf{M}$, a linear simplex $a^{*}$ in some Euclidean space and a simplicial isomorphism $f_{a}: a \rightarrow a^{*}$ such that whenever $b<a$, then the induced simplicial isomorphism $b^{*} \rightarrow f_{a}(b)$ is an isometry. (A map between linear simplexes in Euclidean spaces is an isometry if it extends to an isometry between the affine spaces generated by the simplexes.) If $L$ is a subdivision of $\mathbf{M}$, then $\mathbf{L}$ can be metrized in a natural way. Let $\mathbf{M}^{\prime}$ be another complex, metrized by $\left\{f_{c}^{\prime}\right\}$. $\mathbf{M}$ and $\mathbf{M}^{\prime}$ are isometric if they have subdivisions $\mathbf{L}$ and $\mathbf{L}^{\prime}$ and if there is a simplicial isomorphism $h: \mathbf{L} \rightarrow \mathbf{L}^{\prime}$ such that for every $a \in \mathbf{L}, h$ is an isometry between $a$ and $h(a)$ (more precisely, if $f_{h(a)}^{\prime} \circ h \circ\left(f_{a}\right)^{-1}: a^{*} \rightarrow(h(a))^{*}$ is an isometry, as just defined). An entity associated to a metrized complex $\mathbf{M}$ is intrinsic if it depends only on the isometry class of M. The most important such entity is the "intrinsic metric", which I now describe, following Gluck [4].

Let $\mathbf{M}$ be a metrized complex, let $P, Q \in \mathbf{M}$ and let $\mathbf{J}$ be a closed interval $[s, t] \subseteq \mathbf{R}$. A path from $P$ to $Q$ in $\mathbf{M}$ is a continuous map $\alpha: J \rightarrow \mathbf{M}$ such that $\alpha(s)=P, \alpha(t)=Q$. Unless otherwise stated, every path will have domain $\mathbf{I}$. The space of paths in $\mathbf{M}$ can be given the $C^{0}$ topology; so when one path is said to be "close" to another, it will always mean "pointwise close".

Let $\alpha$ be a p.l. path in $\mathbf{M}$. Let $\mathbf{I}^{\prime}$ be a subdivision of $\mathbf{I}$ such that $\alpha$ maps each 1 -simplex of $\mathbf{I}^{\prime}$ linearly into some simplex of $\mathbf{M}$. For each 1 -simplex $c \in \mathbf{I}^{\prime}$, let $L(\alpha \uparrow c)$ be the length of $f_{a}(\alpha(c))$, where $a \in M$ contains $\alpha(c)$; then $L(\alpha \uparrow c)$ does not depend on the choice of $a$. The length of $\alpha$, denoted $L(\alpha)$, is defined to be $\Sigma\left\{L(\alpha \uparrow c)\right.$ for all 1-simplexes $\left.c \in I^{\prime}\right\}$; this does not depend on the choice of I'. Gluck shows how to define the length of a continuous path $\alpha$ in $\mathbf{M}$ by approximating $\alpha$ by p.l. paths. The precise definition is not important for the purposes of this paper, because from Proposition 2.1 onward I shall use only pl. paths. The intrinsic metric on $\mathbf{M}$, denoted $\rho$ or, when necessary, $\rho_{\mathbf{M}}$, is defined thus: for any $P, Q \in \mathbf{M}, \rho(P, Q)=$ g.l.b. $\{L(\alpha)$ for all paths $\alpha$ from $P$ to $Q\}$; since $M$ was assumed to be connected, this definition makes sense.

A path $\alpha$ from $P$ to $Q$ is minimal if it is parametrized proportionally to arc-length and if $L(\alpha)=\rho(P, Q)$-so that $\alpha$ is as short as possible. Such a path is clearly one-to-one. I shall often refer to im $\alpha$ as a "minimal path"; if $\alpha$ is assumed to have domain $I$, then $\alpha$ is uniquely determined by its image and a choice of initial endpoint, so I doubt that this usage will cause serious ambiguity. A geodesic is a path $\alpha$ which is locally minimal; that is, every $t \in I$ has a neighbourhood $[s, u] \subseteq I$ such that $\alpha \uparrow[s, u]$ is a minimal path. (It follows that $\alpha$ is parametrized proportionally to arc-length.)

Gluck proves these facts: 
Proposition 2.1. (1) If im $\alpha$ is not a subpolyhedron of $\mathbf{M}$, then $\alpha$ can be approximated arbitrarily closely by p.l. paths $\beta$ with the same endpoints, such that $L(\beta) \leqslant L(\alpha)$.

(2) If im $\alpha$ is p.l. but $\alpha$ is not p.l., then $\alpha$ can be approximated arbitrarily closely by p.l. paths $\beta$ with the same endpoints, such that $\beta$ is a re-parametrization of $\alpha$ (so $\operatorname{im} \beta=\operatorname{im} \alpha$ ) and $L(\beta) \leqslant L(\alpha)$.

(3) There is a subdivision $\mathbf{L}$ of $\mathbf{M}$ such that whenever $P, Q$ lie in a simplex $a \in \mathrm{L}$, then the straight-line segment in the simplex between them is the unique minimal path from $P$ to $Q$.

(4) If $\mathbf{M}$ is complete with respect to $\rho$, in particular if $\mathbf{M}$ is a finite complex, then there is at least one minimal path between any two points of $\mathbf{M}$.

The proposition shows that $\rho$ can be defined using only p.l. paths, and hence $\rho$ is indeed intrinsic. Therefore the property of completeness is intrinsic. A metrized complex in which assertion (3) holds will be called a metric complex. Henceforth I shall use only complete metric complexes. When a minimal path from $P$ to $Q$ lies in a simplex, I shall often denote it $P-Q$. The proposition also shows that minimal paths, and hence geodesics, are p.l. Thus to study the intrinsic metric and geodesics one need normally use only p.1. paths; henceforth paths will be pl. unless otherwise stated.

Among p.l. paths, geodesics can be characterized in terms of the "energy function": if $\alpha$ is p.l., let $L(\alpha, t)$ be the arc-length function along $\alpha$; that is, $L(\alpha, t)=L(\alpha \uparrow[0, t])$; and define the energy of $\alpha$ by

$$
E(\alpha)=\int_{0}^{1}\left[\frac{d}{d t} L(\alpha, t)\right]^{2} d t
$$

the derivative here is defined for almost all $t$. Then $E(\alpha) \geqslant[L(\alpha)]^{2}$, and equality holds if and only if $\alpha$ is parametrized proportionally to arc-length. So minimal paths from $P$ to $Q$ are those among $\mathrm{p}$.l. paths from $P$ to $Q$ which minimize energy, and geodesics are paths which do so locally.

I now define "spherical metric complexes", still following Gluck [4]. I am not interested in them for their own sake, but they are useful in studying metric complexes. I offer three reasons. First, many results will be proved by induction on dimension. The natural application of an inductive hypothesis is to the link of a point. But the simplicial link has little relationship to any metric hypotheses. It is more natural to use as the link of a point a sphere of suitably small radius about that point; this sphere is a spherical metric complex. Second, the simplest way to get at the "angle" between two tangent directions at a point seems to be by measuring the distance between their intersections with some spherical link, using the intrinsic metric of this link. The third, and perhaps most interesting, reason I shall save until after the definitions. 
Let $\mathrm{S}^{n}$ be a sphere of radius $\mathrm{p}$ in $\mathrm{R}^{n+1}$. Let $V_{0}, \ldots, V_{k}$, with $k \leqslant n$, be points which lie in an open hemisphere, and let $a$ be the convex hull in $\mathbf{S}$ of $V_{0}, \ldots, V_{k}$. If the $V_{i}$ are in general position, that is, if $a$ is $k$-dimensional, then $a$ is, by definition, a p-spherical $k$-simplex. The $V_{i}$ are its vertices, and every subset of the $V_{i}$ generates a p-spherical simplex which is a face of $a$. In hopes of reducing confusion between the prefixes I shall always use a bold-face letter for the radius. Observe that if $P, Q \in a$, then there is a unique minimal path in $\mathbf{S}$ from $P$ to $Q$, and it lies in $a$; if $P$ and $Q$ are in a face of $a$, then so is the minimal path.

If $a$ is a p-spherical $k$-simplex in $\mathbf{S}^{n}$, then $[a]$ is an affine $(k+1)$-space in $\mathbf{R}^{n+1}$ which passes through the centre of S. A map $f: a \rightarrow a^{\prime}$ between $\mathrm{p}$-spherical simplexes is an isometry if it extends to an isometry $[a] \rightarrow\left[a^{\prime}\right]$. An abstract simplicial complex $\mathbf{M}$ is given a p-spherical metrization by assigning to each $a \in$ $M$ a p-spherical simplex $a^{*}$ in some Euclidean space and a simplicial isomorphism $f_{a}: a \rightarrow a^{*}$ such that whenever $b<a$, then the induced (abstract) simplicial isomorphism $b^{*} \rightarrow f_{a}(b)$ can be realized by an isometry of p-spherical simplexes.

A path $\alpha: \mathbf{I} \rightarrow \mathbf{M}$ is piecewise geodesic if there is a subdivision $\mathbf{I}^{\prime}$ of I such that for every 1-simplex $c \in \mathbf{I}^{\prime}, \alpha(c)$ is contained in a single simplex of $\mathbf{M}$ and $\alpha \uparrow c$ is geodesic in that simplex. As before, one can use piecewise geodesic paths to define the intrinsic metric on $\mathbf{M}$. One can prove an analogue of Proposition 2.1; hence every geodesic is piecewise geodesic, and if $\mathbf{M}$ is complete, then between any two points there is at least one minimal path. And I map speak of p-spherical metric complexes.

For many purposes metric complexes can be included among p-spherical metric complexes, and so it is convenient to allow $p$ to take the value $\infty$. The phrase "a spherical metric complex" will stand for: "a p-spherical metric complex, for some value of p"; thus spherical metric complexes include metric complexes. To distinguish metric complexes from p-spherical ones I shall always specify of the latter that $p$ is finite, and the former I shall call linear metric complexes.

The third reason for introducing spherical metric complexes of finite radius is that in the linear case there are only finitely many minimal paths between two points (this can be inferred from Proposition 3.4); of course this is not true in the spherical case. Thus in p-spherical metric complexes of finite radius the calculus of variations can be used to a limited extent. This is the nub of the proof of Theorem 3. To use the calculus of variations directly in the linear case one would have to redefine concepts as basic as "conjugacy" and the "index" of a geodesic. The proof of Theorem 1 avoids this issue because it deals with a situation in which one can be sure that "conjugate points"-whatever they are-do not occur.

As I mentioned before, one of the reasons for defining spherical metric 
complexes is to be able to discuss the link of a point in a linear metric complex. Let $\mathbf{M}$ be a $\mathbf{p}$-spherical metric complex and let $P \in \mathbf{M}$. The ball of radius $r$ about $P$ is defined to be $B_{P}(r, M)=\{X \in \mathbf{M}$ such that $\rho(P, X) \leqslant r\}$. Its boundary is $S_{P}(r, \mathrm{M})$, the sphere of radius $r$ about $P$. If $r \leqslant \rho(P, \operatorname{link}(P, \mathrm{M}))$, then $B_{P}(r, \mathrm{M})$ and $S_{P}(r, M)$ are indeed a topological ball and sphere, though for large $r$ this need not be the case. Assuming this inequality satisfied, let me abbreviate $B_{P}(r, \mathrm{M})$ and $S_{P}(r, \mathbf{M})$ to $\mathbf{B}$ and $\mathbf{S}$. Then $\mathbf{S}$ has a natural simplicial structure isomorphic to $\operatorname{link}(P, M)$, which is given by a radial projection from $P$ denoted $\psi: \operatorname{star}(P, M)$ $-\{P\} \rightarrow S$. It follows that $S$ is a $q$-spherical metric complex, where $q=$. $\mathrm{p} \sin (r / \mathrm{p})$ if $\mathrm{p}$ is finite and $q=r$ if $\mathrm{p}=\infty$. Let $\rho, \rho^{\prime}$ and $\rho^{\prime \prime}$ be the intrinsic metrics on $\mathbf{M}, \operatorname{star}(P, \mathbf{M})$ and $\mathbf{S}$ respectively. The connection between them is

Lemma 2.2. Assume that $r \leqslant 1 / 2 \rho(P, \operatorname{link}(P, M))$. Then for all $X, Y \in \mathrm{B}$ different from $P$ :

(1) $\rho(X, Y)=\rho^{\prime}(X, Y)$.

(2) The segments $X-P-Y$ constitute the only minimal path from $X$ to $Y$ in $\operatorname{star}(P, \mathrm{M}) \Leftrightarrow \rho^{\prime \prime}(\psi(X), \psi(Y)) \geqslant \pi \mathrm{q}$.

(3) If $\rho^{\prime \prime}(\psi(X), \psi(Y)) \leqslant \pi \mathrm{q}$, then there is a bijection

$\Psi:$ \{minimal paths from $X$ to $Y$ in $\mathrm{B}$ \} $\rightarrow$ \{minimal paths from $\psi(X)$ to $\psi(Y)$ in $\mathbf{S}\}$

such that $\operatorname{im} \Psi(\alpha)=\psi(\operatorname{im} \alpha)$.

Proof. The first assertion is elementary and I omit its proof.

Let $\psi^{\prime}: \operatorname{star}(P, \mathbf{M})-\{P\} \rightarrow \operatorname{link}(P, M)$ denote radial projection from $P$. Let $\alpha$ be a one-to-one piecewise geodesic path in $\mathbf{S}$ from $\psi(X)$ to $\psi(Y)$. Then $\psi^{\prime}$ carries $\alpha$ into a path in $\operatorname{link}(P, M)$ which can be re-parametrized as a pl. path $\alpha^{\prime}$. Let $\mathbf{K}$ be the join $P * \alpha^{\prime}$; then $\mathbf{K}$ is a p-spherical metric 2-disk, and im $\alpha=\mathbf{K} \cap \mathbf{S}$. Say $\rho(P, X)=s, \rho(P, Y)=t$, and let $\theta$ be the angle of $\mathrm{K}$ at $P$, so $\theta=L(\alpha) / \mathrm{q}$. Since $r \leqslant \pi / 2, P * \alpha$ is convex in $\mathbf{K}$, so the minimal path in $\mathbf{K}$ from $X$ to $Y$ lies in $P * \alpha$. In fact (see Diagram 1) there is a unique such path, say $f(\alpha)$.

(*) $f(\alpha)$ consists of the segments $X-P-Y \Leftrightarrow \theta \geqslant \pi$, and then $L(f(\alpha))=$ $s+t$.

(**) $P \notin f(\alpha) \Leftrightarrow \theta \leqslant \pi ;$ and then

$$
\cos (L(f(\alpha)) / \mathbf{p})=\cos (s / \mathbf{p}) \cos (t / p)+\sin (s / p) \sin (t / p) \cos (L(\alpha) / \mathbf{q})
$$

if $\mathrm{p}$ is finite, and

$$
(L(f(\alpha)))^{2}=s^{2}+t^{2}-2 s t \cos (L(\alpha) / r), \quad \text { if } \mathrm{p}=\infty .
$$

It follows that if $\alpha$ is a minimal path in $\mathrm{S}$, then $f(\alpha)$ is a minimal path in $\operatorname{star}(P, \mathrm{M})$ (observe that $X-P-Y$ is the shortest of the paths from $X$ to $Y$ which pass through $P$ ). This proves assertion (2). 
If $\beta$ is a minimal path in $\operatorname{star}(P, M)$ from $X$ to $Y$ which does not pass through $P$, then $\psi(\beta)$ is defined and can be re-parametrized as a piecewise geodesic path in $\mathbf{S}$; by definition, this is $\Psi(\beta)$. Clearly $f(\Psi(\beta))=\beta$. (**) shows that $\Psi(\beta)$ is a minimal path in $S$ of length $\leqslant \pi q$. Hence $\Psi$ is defined, with inverse $f$; and assertion (3) follows.

Let $\mathbf{M}$ be a spherical metric complex. A subset $\mathbf{X} \subseteq \mathbf{M}$ is convex if any minimal path $\alpha$ whose endpoints are in $\mathbf{X}$ itself lies in $\mathbf{X}$.
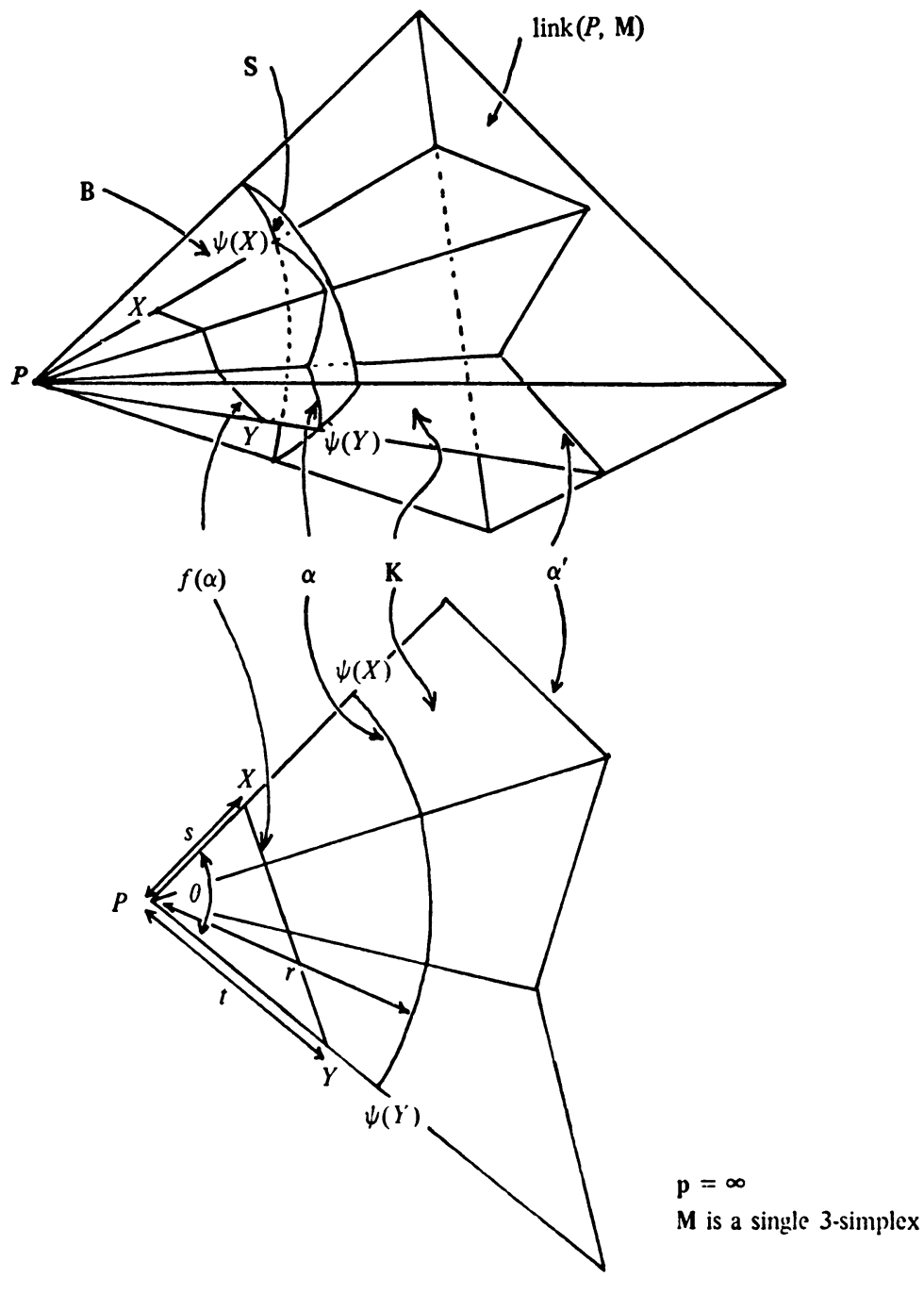

DiAgRAM 1

If in addition $\operatorname{im}(\alpha \uparrow(0,1)) \subseteq$ int $\mathbf{X}$ for every such $\alpha$, then $\mathrm{X}$ is strictly convex. $\mathbf{X}$ is weakly convex if for any two points of $\mathbf{X}$ there is a path between them in $\mathbf{X}$ which is a geodesic in $\mathbf{M}$. A function $f: \mathbf{M} \rightarrow \mathbf{R}$ is convex, strictly convex or 
weakly convex if for every $r \in \mathbf{R}$ the set $f^{r}$, defined as $\{X \in \mathbf{M}$ such that $f(X) \leqslant$ $r\}$, is convex, strictly convex or weakly convex respectively.

Corollary 2.3. If $r \leqslant 1 / 2 \rho(P, \operatorname{link}(P, M))$, then $B_{P}(r, M)$ is strictly convex in $\mathbf{M}$, and $\rho^{\prime}$ is just the restriction of $\rho$ to $\mathbf{B}$.

The metric geometry of a neighbourhood of a point $P$ in a metric complex is determined by the metric geometry of a spherical link of $P$. Lemma 2.2 says roughly that if one is to be told only the structure of geodesics and not the whole intrinsic metric, then it is more valuable to know about geodesics in a link than in a neighbourhood B of $P$; for one does not lose track of geodesics in B which do not pass through $P$ when examining a link of $P$ and one has extra information about geodesics in B which do pass through $P$.

Let $a \subseteq \mathbf{R}^{k}$ and $b \subseteq \mathbf{R}^{l}$ be linear simplexes. Give $a \times b$ the metric in which its factors are orthogonal, so that

$$
\left[\rho_{a \times b}\left(\left(X_{1}, Y_{1}\right),\left(X_{2}, Y_{2}\right)\right)\right]^{2}=\left[\rho_{a}\left(X_{1}, X_{2}\right)\right]^{2}+\left[\rho_{b}\left(Y_{1}, Y_{2}\right)\right]^{2} .
$$

Then $a \times b$ is a convex linear cell in $\mathbf{R}^{k+l}$, and after subdivision can be regarded as a metric complex. Now let $\mathbf{M}$ and $\mathbf{N}$ be linear metric complexes. The linear metric complex $\mathbf{M} \times \mathbf{N}$, called the orthogonal product of $\mathbf{M}$ and $\mathbf{N}$, is defined by metrizing some simplicial subdivision of the cell complex $\mathbf{M} \times \mathbf{N}$ so that for every $a \in \mathbf{M}$ and $b \in \mathbf{N}, a \times b$ is given the orthogonal product metric. That this metrization of $\mathbf{M} \times \mathbf{N}$ is indeed a metric complex follows from this lemma, whose proof I omit:

LEMMA 2.4. A path in $\mathbf{M} \times \mathbf{N}$ is minimal (or is a geodesic) $\Leftrightarrow$ its projections into $\mathbf{M}$ and $\mathbf{N}$ are both minimal (or both geodesics).

It is clear how to generalize the foregoing discussion and Lemma 2.4 to the orthogonal product of any finite number of linear metric complexes.

Let $\mathbf{S}^{l}$ be a sphere of radius $\mathrm{p}$ in $\mathbf{R}^{l+1}$, let $a$ be a $\mathrm{p}$-spherical simplex in $\mathbf{S}$, and let $P \in a$. For each geodesic $\alpha$ from $P$ in $a$, let $D_{P}(\alpha)$ be its tangent vector at $P$. The tangent cone of $a$ at $P$ is defined to be $T_{P}(a)=\bigcup\left\{D_{P}(\alpha)\right.$ for all such $\alpha$ \}; then $T_{P}(a)$ is an unbounded convex linear cone with vertex $P$ lying in the tangent space $T_{P}(\mathbf{S})$ of $\mathbf{S}$ at $P$. Let $b$ be the face of $a$ such that $P \in$ int $b$; then $T_{P}(b)=[b]$, the affine space in $\mathbf{R}^{l+1}$ generated by $b . T_{P}(b)$ is called the base or flat part of $T_{P}(a)$; it can be characterized as $\left\{X \in T_{P}(a)\right.$ such that $T_{P}(a)$ is a cone with vertex $X$ \}.

Now let $\mathbf{M}$ be a spherical metric complex and let $P \in \mathbf{M}$. For each $a \in \mathbf{M}$ such that $P \in a$ I have $T_{P}(a)$ (or more precisely, $T_{f(P)}\left(a^{*}\right)$, where $f: a \rightarrow a^{*}$ is given by a metrization of $\mathbf{M})$. The tangent space $T_{P}(\mathbf{M})$ to $\mathbf{M}$ at $P$ is formed from the disjoint union $\bigcup\left\{T_{P}(a)\right.$ for all $a$ containing $\left.P\right\}$ by making identifications 
according to the natural embeddings $T_{P}(b) \hookrightarrow T_{P}(a)$ whenever $P \in b$ and $b<a$. $T_{P}(\mathrm{M})$ can be given the structure of a linear metric complex. It is intrinsic to $\mathbf{M}$ in the sense that any isometry $h: \mathbf{M} \rightarrow \mathbf{M}^{\prime}$ induces a natural isometry $T_{P}(\mathbf{M})$ $\rightarrow T_{n(P)}\left(\mathrm{M}^{\prime}\right)$.

If $X \neq P$ is in $\operatorname{star}(P, M)$, then $T_{P}(P-X)$ is an infinite ray from $P$. It will be denoted $P \bar{X}$ and called a tangent direction to $\mathrm{M}$ at $P$. Since $T_{P}\left(T_{P}(\mathrm{M})\right)$ can be identified with $T_{P}(\mathrm{M})$, I shall also speak of the tangent direction $P \bar{X}$ when $X \neq P$ is in $T_{P}(\mathrm{M})$. If $\alpha$ is a path from $P$ in either $\mathrm{M}$ or $T_{P}(\mathrm{M})$, then $P \bar{\alpha}$ will denote $P \bar{X}$ where $P-X$ is an initial segment of $\alpha$ in $\operatorname{star}(P, \mathbf{M})$ or $T_{P}(\mathbf{M})$. Similarly if $\alpha$ is a path through $P$, then $P \bar{\alpha}_{+}$and $P \alpha_{-}$will denote the tangent directions to $\alpha$ at $P$ in the directions of increasing and decreasing parameter respectively.

Let $S_{P}(\mathrm{M})$ be the unit sphere about $P$ in $T_{P}(\mathrm{M})$, and let $\rho_{S}$ be the intrinsic metric on $S_{P}(\mathrm{M})$. Let $P \bar{X}, P \bar{Y} \subseteq T_{P}(\mathrm{M})$, and set $X^{\prime}=P \bar{X} \cap S_{P}(\mathbf{M}), Y^{\prime}=P \bar{Y} \cap$ $S_{P}(\mathrm{M})$. The angle between $P \bar{X}$ and $P \bar{Y}$ is defined to be $L \bar{X} P \bar{Y}=\rho_{S}\left(X^{\prime}, Y^{\prime}\right)$. If $\mathbf{N}$ is a subcone of $T_{P}(\mathbf{M})$, then its orthogonal complement is defined to be $\bigcup\{P \bar{Y}$ such that $\angle \bar{X} P \bar{Y}=\pi / 2$ for all $P \bar{X} \subseteq \mathrm{N}\}$.

For example if $r \leqslant 1 / 2 \rho(P, \operatorname{link}(P, \mathbf{M}))$ and if $Q \in S_{P}(r, \mathbf{M})$, then $T_{Q}\left(S_{P}(r, M)\right)$ can be naturally identified with the orthogonal complement of $Q \bar{P}$ in $T_{Q}(\mathbf{M})$. (This is clearly true when $\mathbf{M}$ is a single simplex; one need only add that in general the natural identifications defined for each simplex of $\operatorname{star}(P, M)$ respect the combinatorial structure of $\mathbf{M}$.)

To connect properties of geodesics in $T_{P}(\mathbf{M})$ with those of geodesics in $\mathbf{M}$ near $P$, I now introduce the "exponential map" at $P$. Let $r$ be a number such that $0 \leqslant r \leqslant \rho_{M}(P, \operatorname{link}(P, M))$. Say $\mathbf{M}$ is a $\mathbf{p}$-spherical metric complex. For each $a \in M$ such that $P \in a$, think of $a$ as a simplex in a p-sphere $S^{l} \subseteq R^{l+1}$. Then the standard exponential map $\exp _{P}: T_{P}(\mathrm{~S}) \rightarrow \mathrm{S}$ restricts to a homeomorphism $\exp (a)_{P}: B_{P}\left(r, T_{P}(a)\right) \rightarrow B_{P}(r, a)$. The exponential map at $P, \exp _{P}: B_{P}\left(r, T_{P}(\mathrm{M})\right)$ $\rightarrow B_{P}(r, \mathbf{M})$ is defined to be $\bigcup\left\{\exp (a)_{P}\right.$ for all $a$ containing $\left.P\right\}$. Of course if $\mathbf{M}$ is a linear metric complex, then $\exp _{P}$ is an isometry; the purpose of the next lemma is to show that even if $\mathrm{p}$ is finite, $\exp _{P}$ is still "approximately" an isometry.

Let $\mathrm{B}(r), \mathrm{B}_{T}(r)$ denote $B_{P}(r, \mathrm{M})$ and $B_{P}\left(r, T_{P}(\mathrm{M})\right)$ respectively, and let $\mathrm{S}(r)$ and $\mathrm{S}_{T}(r)$ be defined similarly. Let $\psi: \mathrm{B}(r)-\{P\} \rightarrow \mathrm{S}(r)$ and $\psi_{T}: \mathrm{B}_{T}(r)-$ $\{P\} \rightarrow \mathrm{S}_{T}(r)$ denote radial projection from $P$.

Lemma 2.5. Let $r \leqslant 1 / 2 \rho(P, \operatorname{link}(P, M))$. Then

$(1) \exp _{P}: S_{T}(r) \rightarrow S(r)$ gives a bijection between minimal paths (and hence between geodesics), and preserves angles.

(2) $\exp _{P}: \mathrm{B}_{T}(r) \rightarrow \mathrm{B}(r)$ preserves the structure of geodesics near $P$ in the sense that for any $Q, R \neq P$ in $\mathrm{B}_{T}(r)$ :

(i) The minimal path from $Q$ to $R$ is $Q-P-R \Leftrightarrow$ that from $\exp _{P}(Q)$ to $\exp _{P}(R)$ is $\exp _{P}(Q)-P-\exp _{P}(R)$; in this case $\exp _{P}$ takes one minimal path into 
the other and they have the same length.

(ii) If minimal paths from $Q$ to $R$ do not pass through $P$, then there is a bijection $E:$ \{minimal paths $\gamma_{T}$ from $Q$ to $R$ \} $\rightarrow$ \{minimal paths from $\exp _{P}(Q)$ to $\left.\exp _{P}(R)\right\}$ such that $\operatorname{im} \psi\left(E\left(\gamma_{T}\right)\right)=\exp _{P}\left(\operatorname{im} \psi_{T}\left(\gamma_{T}\right)\right)$; in this case $L\left(\dot{\gamma}_{T}\right)=$ $L\left(E\left(\gamma_{T}\right)\right)+O\left(r^{2}\right)$.

Proof. For linear metric complexes the lemma is trivial, so I assume $\mathbf{M}$ is $\mathrm{p}$-spherical with $\mathrm{p}$ finite. For any $s \geqslant 0$ define the dilation $s_{*}: T_{P}(\mathrm{M}) \rightarrow T_{P}(\mathrm{M})$ with factor $s$ by: $s_{*}(P)=P$, and for $X \neq P, s_{*}(X) \in P \bar{X}$ at distance $s \rho_{T}(P, X)$ from $P$. Clearly $s_{*}$ preserves minimal paths and angles, and multiplies distances by $s$. Hence $s_{*}$ induces a map $s^{\prime \prime}: S_{T}\left(r^{\prime}\right) \rightarrow S_{T}\left(s r^{\prime}\right)$ for any $r^{\prime}$. $s^{\prime \prime}$ also multiplies lengths by $s$, and so preserves geodesics. I claim that $s^{\prime \prime}$ preserves angles. For if $Q \in \mathrm{S}_{T}\left(r^{\prime}\right)$, then $T_{Q}\left(\mathrm{~S}_{T}\left(r^{\prime}\right)\right)$ can be identified with the orthogonal complement $\mathbf{N}_{Q}$ of $Q \bar{P}$ in $T_{Q}\left(T_{P}(\mathrm{M})\right)$, and the tangent map $D s^{\prime \prime}: T_{Q}\left(\mathrm{~S}_{T}\left(r^{\prime}\right)\right) \rightarrow T_{s_{*}(Q)}\left(\mathrm{S}_{T}\left(s r^{\prime}\right)\right)$ with the restriction of the tangent map $D s_{*}: \mathbf{N}_{Q} \rightarrow \mathrm{N}_{s_{*}(Q)}$. But since $T_{P}(\mathrm{M})$ is a linear metric complex, $D s_{*}$ can be identified with $s_{*}$ in a neighbourhood of $Q$. Now $s_{*}$ preserves angles in $\mathrm{N}_{Q}$; hence $s^{\prime \prime}$ does so at $Q$.

Set $r^{\prime}=\mathrm{p} \sin (r / \mathrm{p})$ and $s=r / r^{\prime}$. Then $\exp _{P} \circ s^{\prime \prime}: S_{T}\left(r^{\prime}\right) \rightarrow \mathrm{S}(r)$ is an isometry. Since $\exp _{P}: S_{T}(r) \rightarrow S(r)$ can be written as $\left(\exp _{P} \circ s^{\prime \prime}\right) \circ(1 / s)^{\prime \prime}$, assertion (1) follows.

In fact this argument shows also that $\exp _{P}: S_{T}(r) \rightarrow S(r)$ multiplies all distances by $1 / s$. Part (i) of assertion (2) now follows from Lemma 2.2. That lemma also implies that either both $\Psi$ applied to $\exp _{P}(Q)$ and $\exp _{P}(R)$, and the analogous $\Psi_{T}$ applied in $T_{P}(\mathrm{M})$ to $Q$ and $R$, are defined, or else neither is defined. Note that $\exp _{P}\left(\psi_{T}(Q)\right)=\psi\left(\exp _{P}(Q)\right)$ and similarly for $R$. So $E$ can be defined, under the hypotheses of (ii), as the composition

\{minimal paths from $Q$ to $R$ \}

$$
\begin{aligned}
& \left.\underset{\Psi_{T}}{\longrightarrow} \text { \{minimal paths from } \psi_{T}(Q) \text { to } \psi_{T}(R) \text { in } \mathrm{S}_{T}(r)\right\} \\
& \left.\underset{\exp _{P}}{\longrightarrow} \text { \{minimal paths from } \psi\left(\exp _{P}(Q)\right) \text { to } \psi\left(\exp _{P}(R)\right) \text { in } \mathrm{S}(r)\right\} \\
& \left.\underset{\Psi^{-1}}{\longrightarrow} \text { \{minimal paths from } Q \text { to } R \text { in } \mathrm{B}(r)\right\} .
\end{aligned}
$$

The comparison of $L\left(\gamma_{T}\right)$ to $L\left(E\left(\gamma_{T}\right)\right)$ follows from equation (**) of Lemma 2.2 applied in $\mathbf{B}(r)$ and $\mathbf{B}_{T}(r)$, and the fact that $s=1+O\left(r^{3}\right)$. This completes the proof of Lemma 2.5 .

For example, a geodesic in $\mathbf{M}$ can now be characterized as a path $\alpha$ parametrized proportionally to arc-length such that $\angle \overline{\alpha_{-}} \alpha(t) \overline{\alpha_{+}} \geqslant \pi$ for all $t \in(0,1)$.

So far the train of thought runs thus: to study the nature of minimal paths near a point $P$ in a metric complex $\mathbf{M}$ it suffices to study minimal paths in 
$S_{P}(r, \mathrm{M})$ (Lemma 2.2), or even better, in $S_{P}(\mathrm{M})$ (Lemma 2.5). But this is still not good enough, for the following reason: Let $c$ be the simplex of $\mathbf{M}$ such that $P \in$ int $c$ and let $\mathbf{N}$ be the orthogonal complement of $T_{P}(c)$ in $T_{P}(\mathbf{M})$. Then $T_{P}(\mathbf{M})$ is the orthogonal product $T_{P}(c) \times \mathrm{N}$, and since $T_{P}(c)$ is the flat part of $T_{P}(\mathrm{M})$, all the interesting geometry of $\mathbf{M}$ near $P$ is captured by $\mathbf{N}$. However, assume now that $\operatorname{dim} c \geqslant 1$, so that $S_{P}(c) \subseteq S_{P}(\mathbf{M})$ is nonempty. Then for any $X, Y \in \mathbf{N} \cap S_{P}(\mathbf{M})$ there is a path from $X$ to $Y$ in $S_{P}(\mathrm{M})$ of length $\pi$; namely, connect $X$ and $Y$ to a point of $S_{P}(c)$. It follows that $S_{P}(\mathrm{M})$ has diameter $\leqslant \pi$, and this for a reason which has nothing to do with $N$. One is in fact sacrificing the extra information about geodesics through $P$ in $\mathbf{M}$ that was gained in Lemma 2.2 .

It is perhaps tempting to disregard $T_{P}(c)$ and work only with $\mathbf{N}$. But $\mathbf{N}$ is not intrinsic to $M$ at $P$, since $c$ was not intrinsically chosen. To rectify this, consider all possible isometries between $T_{P}(\mathrm{M})$ and the orthogonal product of a Euclidean space and a cone with vertex $P$, such as $h: \mathbf{R}^{k} \times \mathbf{N}(h) \rightarrow T_{P}(\mathbf{M})$. Choose $h$ so that $k$ is as large as possible; of course $\operatorname{dim} c \leqslant k \leqslant \operatorname{dim} \mathbf{M}$. Then the image $\varphi_{P}(M)=h\left(\mathbf{R}^{k} \times P\right)$ is called the base or flat part of $T_{P}(\mathbf{M})$, and the image $\nu_{P}(\mathrm{M})=h(0 \times \mathrm{N}(h))$ is called the normal geometry of $P$ in $\mathrm{M}$. The base $\varphi_{P}(\mathrm{M})$ is intrinsic to $M$, for it can be characterized as $\left\{Q \in T_{P}(M)\right.$ such that $T_{P}(\mathrm{M})$ is a cone with $Q$ as vertex $\}$. Now $\nu_{P}(\mathrm{M})$ can be intrinsically defined as the orthogonal complement of $\varphi_{P}(\mathrm{M})$ in $T_{P}(\mathrm{M})$. Let $\sigma_{P}(\mathrm{M})$ be the unit sphere in $\nu_{P}(\mathrm{M})$ about $P$, and let $\rho_{\sigma}$ be the intrinsic metric on $\sigma_{P}(\mathrm{M})$; then $\rho_{\sigma}(X, Y)=$ $\angle \bar{X} P \bar{Y}$. Information about geodesics in $\mathbf{M}$ near $P$ is best summarized by information about geodesics in $\sigma_{P}(\mathbf{M})$.

Let $X, Y \in \sigma_{P}(\mathbf{M})$. Then $P \bar{Y}$ is in the open cut locus of $P \bar{X}$, denoted $C^{0}(P \bar{X})$, if there are two or more minimal paths from $X$ to $Y$ in $\sigma_{P}(M)$. The cut locus $C(P \bar{X})$ of $P \bar{X}$ is defined to be $\operatorname{cl}\left[C^{0}(P \bar{X})\right]$. It will follow from Lemma 2.8 that if $\mathbf{M}$ is a manifold without boundary and if $\nu_{P}(\mathbf{M}) \neq\{P\}$, then $C(P \bar{X}) \neq \varnothing$ for any $P \bar{X} \subseteq \nu_{P}(M)$. Assuming that $C(P \bar{X}) \neq \varnothing$, the maximum and minimum curvatures of $\mathbf{M}$ at $P$ in the direction $P \bar{X} \subseteq \nu_{P}(\mathbf{M})$ are defined by:

$$
\begin{aligned}
& \kappa_{+}(P \bar{X})=2 \pi-2 \min \{L \bar{X} P \bar{Y} \text { for } P \bar{Y} \subseteq C(P \bar{X})\} \\
& \kappa_{-}(P \bar{X})=2 \pi-2 \max \{L \bar{X} P \bar{Z} \text { for } P \bar{Z} \subseteq C(P \bar{X})\} .
\end{aligned}
$$

If $C(P \bar{X})=\varnothing$, I set $\kappa_{+}(P \bar{X})=\kappa_{-}(P \bar{X})=0$.

ExAmples. 1. Let $\mathbf{M}$ be a linear 2 -manifold without boundary. If $P$ is other than a vertex of nonzero curvature, then $M$ is flat near $P$; that is, $\varphi_{P}(M)=$ $T_{P}(\mathbf{M})$ and $\nu_{P}(\mathbf{M})=\{P\}$, so $\mathbf{M}$ is locally isometric to $\mathbf{R}^{2}$. Diagram 2 shows points of positive and negative curvature. Geodesics from a point $Q$ near $P$, as seen from above, look roughly as in Diagram 3. To determine geodesics from $Q$ exactly it is best to develop the two cones in the plane by cutting each of them open along the ray $P \bar{X}$ "opposite" to $P \bar{Q}$; then in the resulting planar nets, geodesics will be 
straight lines wherever possible (see Diagram 4). Observe that $F \bar{X}$ is just the cut locus of $P \bar{Q}$ (once $M$ is identified with $T_{P}(M)$ near $P$ ). In this example $\kappa_{+}(P \bar{Q})$ $=\kappa_{-}(P \bar{Q})=2 \pi-2 \angle \bar{Q} P \bar{X}$, which is indeed the usual 2-dimensional curvature of $M$ at $P$. In this case the curvature does not depend on $P \bar{Q}$, only on $P$.

2. The proof of Theorem 4 hinges on this example: let $\mathbf{M}$ be a simplicial 3-disk triangulated as the join of a 1-simplex $c$ to a pentagon $\mathbf{N}$, so $\mathbf{M}$ consists of five 3-simplexes about a 1-simplex. $\mathbf{M}$ is metrized by making all the 1 -simplexes have length 1 . $M$ is actually a metric complex because it can be linearly embedded in $\mathbf{R}^{4}$. Let $P$ be the midpoint of $c$. Then $\varphi_{P}(\mathbf{M})$ is essentially $c$ and $\nu_{P}(\mathbf{M})$ essentially $P * \mathbf{N}$ (at least in a neighbourhood of $P$ ). The total angle of $P * \mathbf{N}$ at $P$ is $\leqslant 2 \pi$. Again $\kappa_{+}(P \bar{X})=\kappa_{-}(P \bar{X})=2 \pi-($ total angle of $P * N$ at $P$ ) does not depend on the choice of $P \bar{X} \subseteq \nu_{P}(M)$, and the curvature at $P$ is $\leqslant 0$.

3. More generally if $\mathbf{M}$ is a metric complex (linear or spherical) which is an $n$-manifold without boundary, then $\kappa_{+}(P \bar{X})=\kappa_{-}(P \bar{X})=0$ whenever $P \in$ int $a$ with $\operatorname{dim} a \geqslant n-1$. If $\operatorname{dim} a=n-2$, then either $\nu_{P}(\mathbf{M})=\{P\}$, in which case $M$ is flat near $P$, or else $\operatorname{dim} \nu_{P}(M)=2$. In this case $\kappa_{+}(P \bar{X})=\kappa_{-}(P \bar{X})$ and the curvature is independent of the direction $P \bar{X} \subseteq \nu_{P}(\mathrm{M})$.
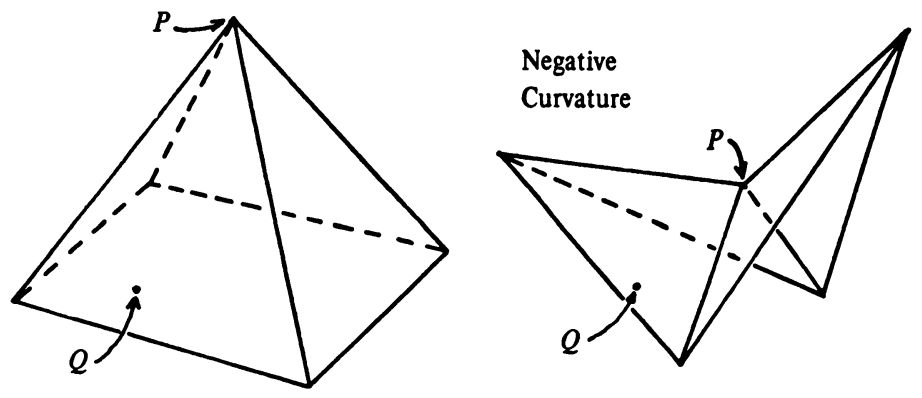

Positive curvature

Diagram 2
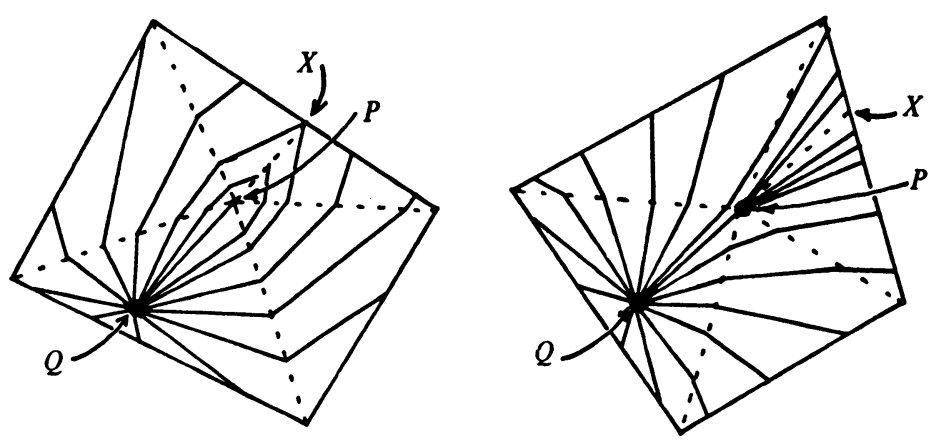

Diagram 3 

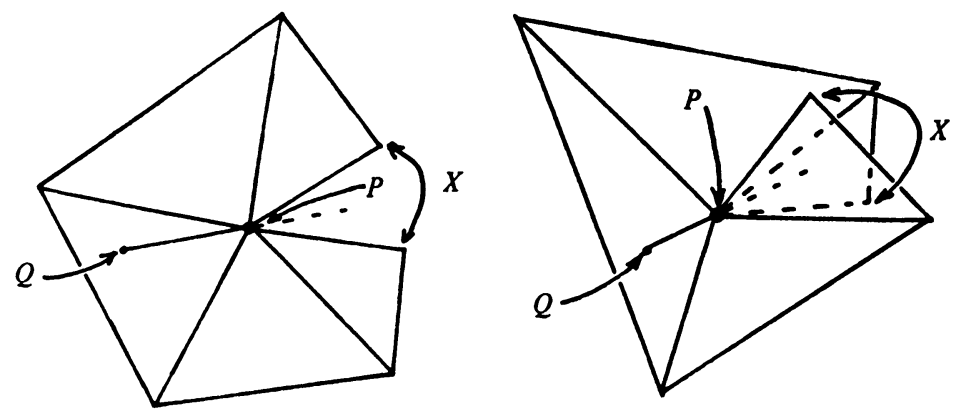

\section{Diagram 4}

4. Here is an example in which the curvature at some points does depend on which direction one chooses. Let $U$ be the (closed) exterior of a unit cube in $\mathbf{R}^{\mathbf{3}}$ with boundary $\mathbf{C}$, and let $\mathbf{V}$ be a copy of $\mathbf{U}$. Form $\mathbf{M}$ from the disjoint union $\mathbf{U} \cup \mathbf{V}$ by identifying bdy $\mathbf{U}$ to bdy $\mathbf{V}$ in the obvious way. Clearly the geometry is interesting only at the edges and vertices of $\mathbf{C}$. If $c$ is an edge of $\mathbf{C}$ and $P \in$ int $c$, then $\nu_{P}(M)$ is 2-dimensional, so the curvatures at $P$ are equal and do not depend on the direction used. In fact $\kappa_{+}(P \bar{X})=\kappa_{-}(P \bar{X})=-\pi$ in all directions. However, the situation is different if $P$ is a vertex of $C$. Now $\varphi_{P}(M)$ $=\{P\}$ and $\nu_{P}(\mathrm{M})=T_{P}(\mathrm{M})$. Let $P \bar{X}$ be the axis of symmetry of $\mathrm{U}$ at $P$, and $P \bar{Y}$ the corresponding direction in V. Then $P \bar{Y} \subseteq C(P \bar{X})$ and maximizes $\angle \bar{X} P \bar{Y}$. It follows that $\kappa_{-}(P \bar{X})=4 \tan ^{-1}\left(2^{1 / 2}\right)-2 \pi \leqslant 0$. Also $\kappa_{+}(P \bar{X})=0$. These statements are easy to verify once one knows what geodesics from $X$ in $S_{P}(M)$ look like. They are drawn (not very accurately) in Diagram 5 . First I have split $S_{P}(\mathrm{M})$ into $S_{P}(\mathrm{U})$ and $S_{P}(\mathrm{~V})$; each is the complement in the 2-sphere $S_{P}\left(\mathbf{R}^{3}\right)$ of the spherical triangle $E_{1} E_{2} E_{3}=S_{P}$ (unit cube). Then I have projected $S_{P}(\mathrm{U})$ into $\mathbf{R}^{2}$ stereographically from the antipode $X^{\prime}$ of $X$ in $S_{P}\left(\mathbf{R}^{3}\right)$, and treated $S_{P}(\mathrm{~V})$ similarly. Geodesics from $X$ are suggested by dashed lines, which of course continue from one half of the picture into the other. The region $E_{2} E_{3} X_{1}^{\prime \prime}$ represents a spherical triangle such that $X E_{2} E_{3} \cup E_{2} E_{3} X_{1}^{n}$ is isometric to a portion of a 2-sphere in which $X$ and $X_{1}^{\prime \prime}$ are antipodal and $X E_{2} X_{1}^{\prime \prime}$ and $X E_{3} X_{1}^{\prime \prime}$ are great semicircles. In this region geodesics from $X$ all converge to $X_{1}^{\prime \prime}$. The region $X_{1}^{\prime \prime} X_{2}^{\prime \prime} E_{3}$ is isometric to half of a polar cap about $E_{3}$ in a 2-sphere. In this region geodesics from $X$ consist of the geodesic $X-E_{3}$ in $S_{P}(U)$ followed by geodesics from $E_{3}$. The region $X_{1}^{n} X_{2}^{\prime \prime} X_{3}^{\prime \prime}$ represents the points distant more than $\pi$ from $X$. $C(P \bar{X})$ is the infinite cone from $P$ on $\bigcup\left\{Y-X_{1}^{\prime \prime}\right.$ for $\left.i=1,2,3\right\}$. Geodesics from $X$ in the region $Y X_{2}^{\prime \prime} X_{3}^{\prime \prime}$ are the continuations of geodesics from $E_{1}$.

On the other hand $T_{P}(\mathrm{C})$ is geodesically closed in $T_{P}(\mathrm{U})$ and $T_{P}(\mathrm{~V})$, and hence in $T_{P}(\mathrm{M})$. Thus if $Q$ is the midpoint of $E_{2}-E_{3}$ in $S_{P}(\mathrm{M})$, then $P \bar{Q}=$ $C\left(P \overline{E_{1}}\right.$ in $\left.T_{P}(\mathrm{C})\right)$. Let $E_{1}^{\prime}$ be antipodal to $E_{1}$ in $S_{P}\left(R^{3}\right)$. It is not hard to see that 

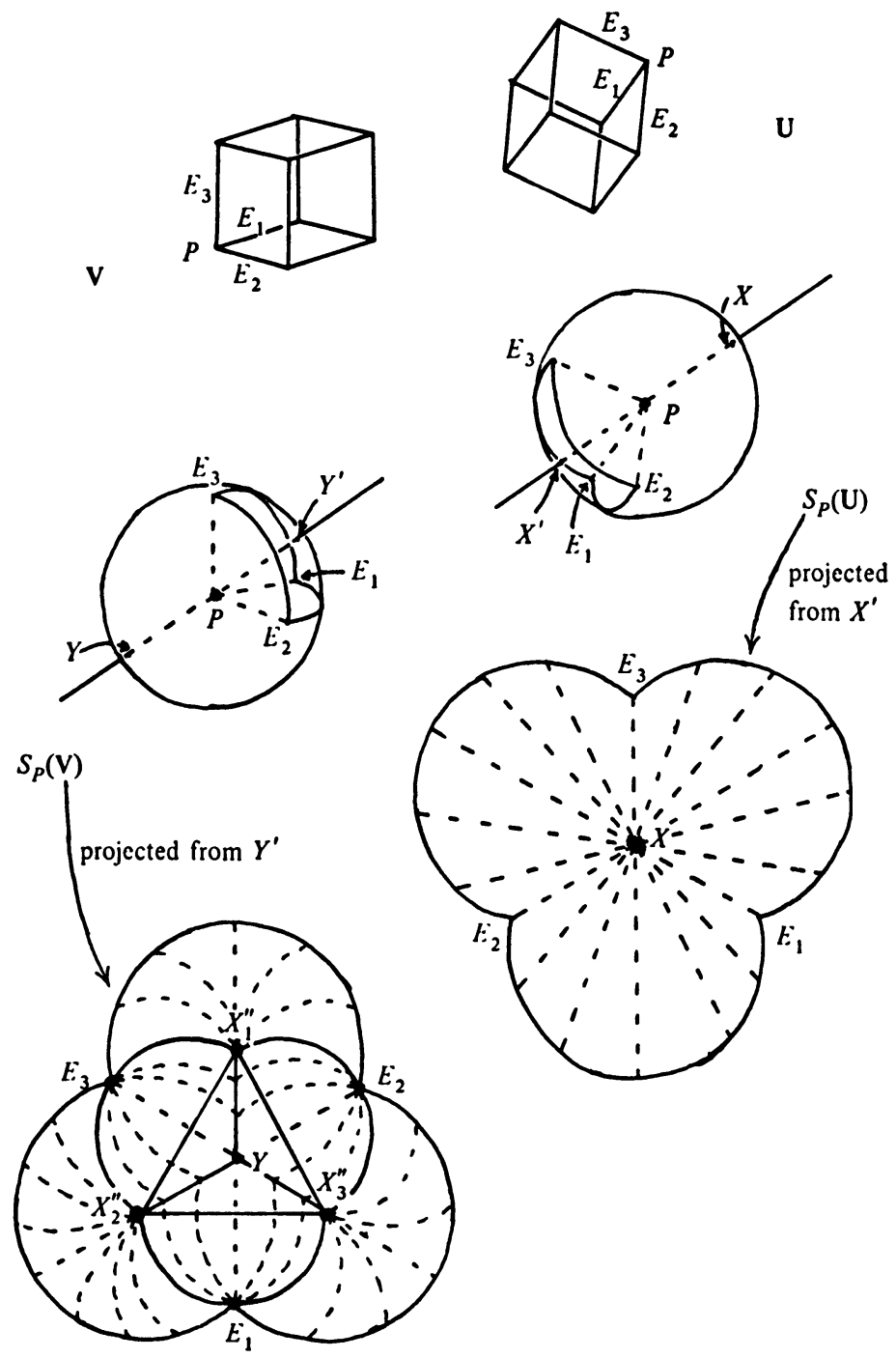

Diagram 5

C $\left(P \overline{E_{1}}\right.$ in $\left.T_{P}(\mathrm{U})\right)$ is the infinite cone from $P$ on $Q-E_{1}^{\prime}$. It follows that $\kappa_{-}\left(P \overline{E_{1}}\right)=$ 0 and $\kappa_{+}\left(P \overline{E_{1}}\right)=\pi / 2$. Thus the curvature hypothesis of Theorem 1 is not satisfied by $M$; which is just as well, since $M$ is homeomorphic to $S^{2} \times R^{1}$.

I shall say that a spherical metric complex $\mathbf{M}$ has unique minimal paths near $P$ if $P$ has a neighbourhood $\mathrm{B}$ in $\mathbf{M}$ which is convex and such that for every $X, Y$ $\in \mathrm{B}$ there is a unique minimal path from $X$ to $Y$ in $\mathbf{B}$. $M$ has unique minimal paths locally if this is true for every $P \in M$. If for every $X, Y \in M$ there is a unique minimal path (or geodesic) from $X$ to $Y$, then $M$ has unique minimal paths (or geodesics). 
LEMma 2.6. Let $\mathbf{M}$ be $\mathrm{p}$-spherical and let $P \in \mathbf{M}$. Then these conditions are equivalent:

(1) $\kappa_{+}(P \bar{X}) \leqslant 0$ for all $P \bar{X} \subseteq \nu_{P}(\mathbf{M})$;

(2) $\mathbf{M}$ has unique minimal paths near $P$;

(3) if $r \leqslant 1 / 2 \rho(P, \operatorname{link}(P, \mathrm{M}))$ and if $\mathrm{q}=\mathrm{p} \sin (r / \mathrm{p})$, then whenever $X, Y \in$ $S_{P}(r, \mathrm{M})$ with $\rho_{S}(X, Y) \leqslant \pi \mathrm{q}$, there is a unique minimal path from $X$ to $Y$ in $\mathbf{S}$.

Proof. Condition (1) holds $\Leftrightarrow v_{P}(\mathrm{M})$ has unique minimal paths (by Lemma $2.2) \Leftrightarrow T_{P}(\mathrm{M})$ does so (by Lemma 2.4) $\Leftrightarrow B_{P}\left(r, T_{P}(\mathrm{M})\right.$ ) does so (by Corollary 2.3) $\Leftrightarrow B_{P}(r, M)$ does so (by Lemma 2.5); and this is just condition (2). Conditions (2) and (3) are equivalent by Lemma 2.2 again.

Lemma 2.7. Let $\mathrm{X}$ and $\mathrm{Y}$ be subsets of a spherical metric complex $\mathbf{M}$. Assume that for every $X \in \mathrm{X}$ and $Y \in \mathrm{Y}$ there is a unique minimal path in $\mathbf{M}$ from $X$ to $Y$. Then these minimal paths vary continuously (pointwise) with their endpoints.

Proof. Let $\alpha_{i}$ be the minimal path from $X_{i} \in \mathrm{X}$ to $Y_{i} \in \mathrm{Y}$, where $X_{i} \rightarrow$ $X \in \mathrm{X}, Y_{i} \rightarrow Y \in \mathrm{Y}$, and let $\alpha$ be the minimal path from $X$ to $Y$. The $\alpha_{i}$ are eventually all contained in some compact set (for example, $B_{X}(2 \rho(X, Y), \mathbf{M})$ ). Hence the $\alpha_{i}$ have (pointwise) convergent subsequences. The limit of any such subsequence is a path from $X$ to $Y$ whose length is $\rho(X, Y)$; that is, the limit path is a minimal path from $X$ to $Y$; it must therefore be $\alpha$. This proves the lemma.

LEMMA 2.8. Let $\nu_{P}(\mathrm{M})$ be the normal geometry of $P$ in a spherical metric complex $\mathbf{M}$ which is a manifold without boundary. Assume $\nu_{P}(\mathbf{M}) \neq\{P\}$. Let $P \bar{X} \subseteq \nu_{P}(\mathbf{M})$, and let $P \bar{Y} \subseteq \nu_{P}(\mathbf{M})$ be such that $\angle \bar{X} P \bar{Y}$ is as large as possible. Then $P \bar{Y} \subseteq C(P \bar{X})$.

Proof. Say $P \bar{X}$ and $P \bar{Y}$ meet $\sigma_{P}(\mathbf{M})$ in $X$ and $Y$. Suppose $P \bar{Y} \nsubseteq C(P \bar{X})$. Set $\mathbf{B}=B_{Y}\left(r, \sigma_{P}(\mathbf{M})\right)$ and $\mathbf{S}=$ bdy $\mathbf{B}$. Then for some $r \geqslant 0$ there is a unique minimal path $\alpha_{Z}$ from each point $Z \in \mathbf{B}$ to $X$. By Lemma $2.7 \alpha_{Z}$ varies continuously in $Z$. So $\bigcup\left\{\alpha_{Z}\right\}$ defines a map $f: X * \mathbf{B} \rightarrow \sigma_{P}(\mathbf{M})$, a homotopy of $\mathbf{B}$ down to $X$. But for $Z \in \mathrm{S}, \alpha_{Z}$ does not pass through $Y$. So $Y \notin f(X * \mathrm{~S})$; this is a contradiction, and the lemma is proved.

Corollary 2.9. $\kappa_{-}(P \bar{X}) \supsetneqq 0$ for all $P \bar{X} \subseteq \nu_{P}(M) \Leftrightarrow \operatorname{diam} \sigma_{P}(M) \preccurlyeq \pi$.

These conditions are also equivalent, as will be shown in Lemma 5.1, to saying that if a geodesic $\alpha$ passes through $P$, then $P \overline{\alpha_{+}}$and $P \overline{\alpha_{-}}$lie in $\varphi_{P}(\mathbf{M})$.

3. The hypothesis of unique minimal paths. This section is given to the proof of some results needed in $\S 4$, which is about metric complexes of negative 
curvature. Throughout this section, unless otherwise stated, I shall hypothesize of any $\mathbf{p}$-spherical metric complex $\mathbf{M}$ that whenever $Q, R \in \mathbf{M}$ with $\rho(Q, R) \leqslant$ $\pi \mathrm{p}$, there is a unique minimal path from $Q$ to $R$. The main examples used in $\S 4$ are these: Let $\mathbf{M}^{\prime}$ be a spherical metric complex and let $P \in \mathbf{M}$ be such that $\kappa_{+}(P \bar{X}) \leqslant 0$ for all $P \bar{X} \subseteq \nu_{P}(M)$. Then, for any $r \leqslant 1 / 2 \rho(P, \operatorname{link}(P, M)), B_{P}(r, M)$ and $S_{P}(r, \mathrm{M})$ satisfy the above hypothesis, by Lemma 2.5 .

Propositions 3.1 to 3.5 are the results from this section which will be quoted in $\S 4$; they are stated directly below. The reader who accepts their proof may proceed directly to $\$ 4$.

Proposition 3.1. Let $\mathrm{M}$ be a linear metric complex, and let $\alpha$ and $\beta$ be minimal paths. Then for all $t \in[0,1]$,

$$
\rho(\alpha(t), \beta(t)) \leqslant(1-t) \rho(\alpha(0), \beta(0))+t \rho(\alpha(1), \beta(1)) .
$$

Proposition 3.2. Any geodesic of length $\leqslant \pi \mathrm{p}$ is a minimal path.

Proposition 3.3. Let $P, Q, R \in \mathbf{M}$ be such that $\rho(P, Q), \rho(P, R) \leqslant \pi \mathrm{p} / 2$. Then the minimal path $\gamma$ from $Q$ to $R$ satisfies: $\rho(P, \gamma(t)) \leqslant(1-t) \rho(P, Q)+$ $t \rho(P, R)$, and equality holds only if $t=0$ or 1 , or if $P, Q$ and $R$ lie on a minimal path.

Proposition 3.4. Let $\mathrm{M}$ be a $\mathrm{p}$-spherical metric complex, not necessarily having unique minimal paths. Let $\alpha$ be a geodesic from $P$ to $Q$ of length $\leqslant \pi p$. Then there is an $\epsilon \geqslant 0$ such that whenever $\beta$ is a path from $P$ to $Q$ pointwise closer than $\epsilon$ to $\alpha$ and $\operatorname{im} \beta \neq \operatorname{im} \alpha$, then $L(\beta) \geqslant L(\alpha)$.

Proposition 3.5. Let $P \in \mathbf{M}$ and let $q \leqslant \pi \mathrm{p} / 2$. Then for any simplex $a$, $B_{P}(q, \mathbf{M}) \cap a$ is either empty, a single point, or a smooth, strictly convex body with nonempty interior in a.

I first give a rough outline of the proof of Proposition 3.1 as typical of the methods of this section. It suffices to prove the proposition in case $\alpha(0)=\beta(0)$ $=P$, say (Lemma 3.9). Let $\gamma$ be the minimal path from $Q=\alpha(1)$ to $R=\beta(1)$, and for each $t$ let $\alpha_{t}$ be the minimal path from $P$ to $\gamma(t)$. The paths $\alpha_{t}$ vary continuously in $t$, so their union forms a sort of "triangle" $P Q R$ in $M$. This "triangle" is not necessarily polyhedral, but it can be sufficiently well approximated by a polyhedron, so let me assume that $P Q R$ is itself one. To prove the proposition one may consider only $P Q R$ with its intrinsic metric. I distinguish three cases. The proof is simplest in Case I: $P Q R$ has no interior points of curvature and $\gamma$ is a straight-line segment. The "sides" $P Q$ and $P R$ of $P Q R$ need not be straight, but they can have only reflex angles. In this case $P Q R$ can be constructed in $\mathbf{R}^{2}$ (Lemma 3.6) and the required inequality can now be easily 
proved. In Case II, $P Q R$ still has no interior points of curvature, but $\gamma$ is not straight. Then the angle at a vertex $Z$ of $\gamma$ is reflex. Cutting $P Q R$ along the $\alpha_{t}$ 's from $P$ to the various $Z$ 's decomposes $P Q R$ into "triangles" in Case $\mathrm{I}$, and the required inequality follows. In Case III, the general case, $P Q R$ may have interior points of curvature, $Y$. They all have negative curvature; in fact, cutting $P Q R$ along the $\alpha_{t}$ 's from $P$ through the $Y$ 's decomposes $P Q R$ into "triangles" in Case II (Lemma 3.8), and the proposition follows.

Now the points $Y$ arise (roughly speaking) in this way: A generic $\alpha_{t}$ passes through the interiors of simplexes $a_{1}(t), b_{1}(t), a_{2}(t), \ldots, b_{k-1}(t), a_{k}(t)$, in that order, with dimensions alternately $n, n-1, n, \ldots, n$ (assuming that $\gamma$ is also generic). The exceptional $\alpha_{t}$ 's pass through $(n-2)$-simplexes, generically speaking; these $\alpha_{t}$ 's are isolated and meet the $(n-2)$-simplexes in isolated points, which are the $Y$ 's. Thus Case I arises when examining "short" paths $\gamma$ such that the sequence $a_{1}(t), \ldots, a_{k}(t)$ is the same for all $t$. The generic case is not the general case, however; and in general one cannot assume that the $a_{i}(t)$ and $b_{i}(t)$ have dimensions $n$ and $n-1$ respectively, even for most values of $t$. Lemma 3.7 shows that, nonetheless, for short segments of $\gamma$, Case I does apply. To analyze Case I, and also to construct a p.l. approximation to $P Q R$, I first fix a sequence of the form $a_{1}(t), \ldots, a_{k}(t)$ and examine paths which yield this sequence.

Let $\mathbf{M}$ be a spherical metric complex, and let $P, Q \in \mathbf{M}$. A chain from $P$ to $Q$ is a sequence $C=\left(a_{1}, \ldots, a_{k}\right)$ of simplexes of $M$ such that $P \in a_{1}, Q \in$ $a_{k}$ and $b_{i}=a_{i} \cap a_{i+1}$ is nonempty, for $i=1, \ldots, k-1$ (if $k=1$ this condition shall be void). A path $\alpha$ from $P$ to $Q$ lies within $C$ if there are $x_{1}, \ldots, x_{k-1} \in I$ (which need not be distinct) such that $\alpha\left(x_{i}\right)=b_{i}$ and such that (setting $x_{0}=0$, $\left.x_{k}=1\right) \alpha$ maps $\left[x_{i}, x_{i+1}\right]$ into $a_{i+1}$ and $\alpha \uparrow\left[x_{i}, x_{i+1}\right]$ is a geodesic, for $i=0$, $\ldots, k-1$. The development of $C$ is a spherical metric complex $C^{*}$ defined thus: take disjoint simplexes $a_{i}{ }^{*}$ isometric to $a_{i}$, and identify, for $i=1, \ldots, k-1$, the faces corresponding to $b_{i}$ in $a_{i}$ and $a_{i+1}$. Then $C^{*}$ has its intrinsic metric $\rho_{C^{*}}$; however I shall usually write $\rho_{C}$ for $\rho_{C}$, and call $\rho_{C}$ a "metric" on $C$, to save explicit mention of $C^{*}$.

If $P \in a_{1}$ and $Q \in a_{k}$, then any geodesic from $P$ to $Q$ in the metric $\rho_{c}$ is a path within $C$. On the other hand, every geodesic from $P$ to $Q$ in $M$ lies within some chain. For many purposes, including that of looking for minimal paths in $\mathbf{M}$, it would make sense to lay down as an axiom of chains that the simplexes of a chain be distinct; but in the proof of Proposition 3.4 it will be useful to allow chains to have repeated simplexes.

Let paths $\alpha$ and $\beta$ from $P$ to $Q$ and $R$ within $C$ be determined by points $X_{i}$ and $Y_{i}$ of $b_{i}$ respectively, for $i=1, \ldots, k-1$; assume $Q$ and $R$ are both in $a_{k}$. Then I can form these 2-simplexes (some of which may be degenerate): 


$$
\begin{gathered}
\left\langle P, X_{1}, Y_{1}\right\rangle \subseteq a_{1}, \\
\left\langle X_{i-1}, Y_{i-1}, Y_{i}\right\rangle,\left\langle X_{i-1}, X_{i}, Y_{i}\right\rangle \subseteq a_{i} \text { for } i=2, \ldots, k-1,
\end{gathered}
$$

and

$$
\left\langle X_{k}, Y_{k}, Q\right\rangle,\left\langle X_{k}, Q, R\right\rangle \subseteq a_{k} .
$$

The metric 2-complex formed by the union of these simplexes, $K$, is called the span of $\alpha$ and $\beta$. Topologically $\mathbf{K}$ looks like a finite sequence of 2 -disks with consecutive ones either touching at boundary points or being joined by an arc between boundary points.

The next lemma shows that if $\alpha$ and $\beta$ are geodesics and are not too long, then $\mathbf{K}$ is either an arc, a disk, or at most an arc followed by a disk; that is, once $\alpha$ and $\beta$ separate they cannot again converge. In the outline of the proof of Proposition 3.1, $\mathrm{K}$ is the polyhedron that approximates, in Case I, the "triangle" of minimal paths from $P$ to $Q-R$.

LEMMA 3.6. In the previous notation assume that $\alpha$ and $\beta$ are geodesics of length $\leqslant \pi \mathrm{p} / 2$. Then $\mathbf{K}$ is isometric to a metric complex $\mathbf{L}$ in a $\mathrm{p}$-spherical 2-sphere $\mathbf{S}$ of this form:

(1) $L=L_{1} \cup L_{2}$, where $L_{1}$ is a polygonal region and $L_{2}$ an arc;

(2) the boundary of $\mathrm{L}_{1}$ is a simple, closed curve made of geodesic segments with vertices $W, U_{1}, U_{2}, \ldots, U_{m}, V_{n}, \ldots, V_{1}$ in that order;

(3) $\angle U_{1}, \ldots, \angle U_{m-1}, \angle V_{n-1}, \ldots, \angle V_{1}$ (measured within $L$ ) are $¥ \pi$;

(4) the length of $U_{m}-V_{n}$ is $\leqslant \pi \mathrm{p}$;

(5) $\mathrm{L}_{1} \subseteq$ int $B_{W}(\pi \mathrm{p} / 2, \mathrm{~S})$;

(6) $\mathrm{L}_{2}$ is a geodesic segment $W-X$;

(7) $\mathbf{L}_{1} \cap \mathbf{L}_{2}=W$.

(See Diagram 6.) Under this isometry $P, Q$ and $R$ correspond to $X, U_{m}$ and $V_{n}$ respectively, $\alpha$ to $X-W-U_{1}-\cdots-U_{m}$ and $\beta$ to $X-W-V_{1}-\cdots-V_{n}$. Thus $X-W$ represents whatever initial portion im $\alpha$ and im $\beta$ have in common; of course if $\operatorname{im} \alpha \cap \operatorname{im} \beta=P$, then $X=W$ and $\mathrm{L}_{2}$ is degenerate. $\mathrm{L}_{1}$ can also be degenerate.

Proof. Let $c_{1}, \ldots, c_{l}$ in this order be the 2 -simplexes (some of which may be degenerate) used to define $\mathbf{K}$. By discarding for now an initial portion common to $\alpha$ and $\beta$, I may assume $c_{1}$ is nondegenerate. The proof is by induction on $j=1, \ldots, l$; the inductive hypothesis is that $c_{1} \cup \cdots \cup c_{j}$ has been embedded in $\mathbf{S}$ and that its image, which I call by the same name, lies in the nondegenerate, convex, spherical triangle $c_{j}^{\prime}=P * d_{j}$ (where $d_{j}=c_{j} \cap c_{j+1}$ for $j=1, \ldots, l-1)$.

The initial case is trivial. Now let $j=2$. Consider first the possibility that $c_{2}$ is degenerate. It suffices to show that $d_{2}$ is not degenerate. If this did happen, 
say $d_{1}=\left\langle w_{1}^{1}, w_{1}^{2}\right\rangle$ and $d_{2}=w_{1}^{1}$ (observe that $d_{j} \cap d_{j+1}$ can never be empty), then $w_{1}^{2}$ would be an interior vertex of either $\alpha$ or $\beta$, say of $\beta$, and $c_{1}$ would be a neighbourhood of $w_{1}^{2}$ in $\mathrm{K}$. But $\angle w_{1}^{2}$ (in $\mathrm{K}$ ) $\leqslant \pi$ (since $c_{1}$ is a convex triangle in S); this would imply that $\beta$ is not a geodesic in $\mathbf{K}$ at $w_{1}^{2}$, a fortiori not in $\mathbf{M}$, contrary to hypothesis. Now assume $c_{2}$ is nondegenerate. I can embed $c_{2}$ in $\mathbf{S}$ so that a neighbourhood of $d_{1}$ in $c_{1} \cup c_{2}$ is embedded. The vertices of $c_{2}$ are distant $\leqslant \pi \mathrm{p} / 2$ from $P$ in $\mathrm{K}$, and hence in $\mathrm{S}$, since $L(\alpha)$ and $L(\beta) \leqslant \pi \mathrm{p} / 2$. So $c_{2}$ lies in the convex set

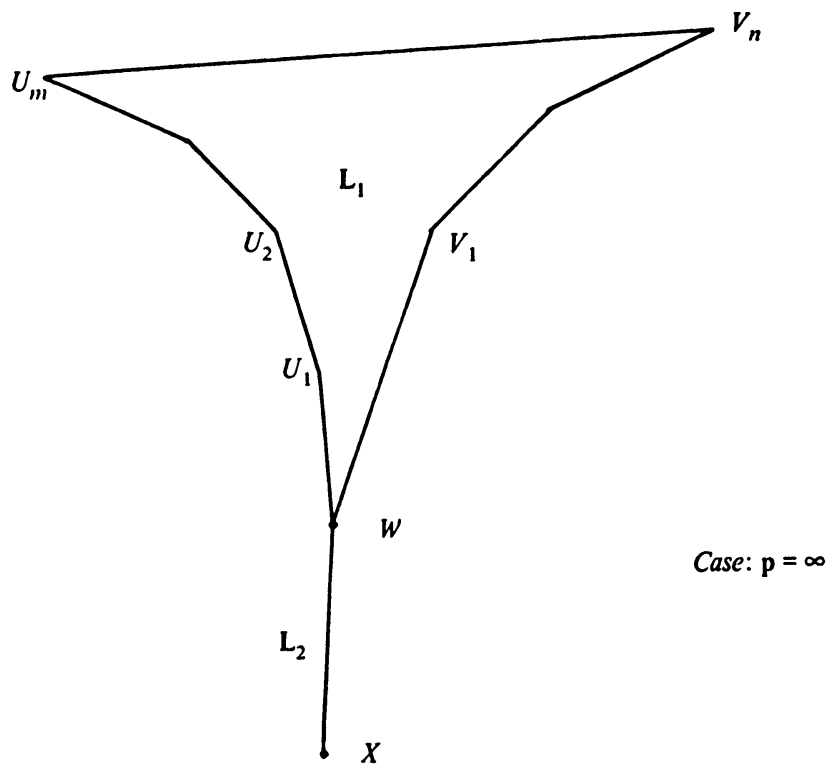

Diagram 6

int $B_{P}(\pi \mathrm{p} / 2, \mathrm{~S})$, and it follows that $c_{1} \cup c_{2}$ is embedded. Say $d_{2}=\left\langle w_{1}^{1}, w_{2}^{2}\right\rangle$, and say $w_{1}^{2}$ is a vertex of $\beta$. For $\beta$ to be a geodesic in $M$, and hence in $K, \angle w_{1}^{2}$ (in K) must be $\geqslant \pi$. Hence $c_{1} \cup c_{2} \subseteq P * d_{2}$. So $c_{2}^{\prime}$ is 2 -dimensional and thus nondegenerate; it is convex because $L\left(d_{2}\right) \leqslant \pi \mathrm{p}$ and its other two sides have length $\leqslant \pi \mathrm{p} / 2$. This finishes the case $j=2$.

For the general step I apply the same argument to $c_{j}^{\prime}$ and $c_{j+1}$, with these modifications: If $c_{j+1}$ is degenerate, let $d_{j}=\left\langle w_{j}^{1}, w_{j}^{2}\right\rangle$; then $\angle w_{j}^{i}$ (in $c_{1} \cup \cdots$ $\cup c_{j}$ ) $\leqslant \angle w_{j}^{i}$ (in $c_{j}^{\prime}$ ) $\leqslant \pi$ (since $c_{j}^{\prime}$ is convex), and it follows that $d_{j+1}$ cannot be degenerate. If $c_{j+1}$ is nondegenerate, say $d_{j+1}=\left\langle w_{j}^{1}, w_{j+1}^{2}\right\rangle$; then $\angle w_{j}^{1}$ (in $\left.c_{j}^{\prime} \cup c_{j+1}\right) \geqslant L w_{j}^{1}($ in $\mathbf{K}) \geqslant \pi$, and as before $c_{j}^{\prime} \cup c_{j+1} \subseteq P * d_{j+1}$. This completes the inductive step.

At the end of the induction $L_{1}$ has been constructed but for labelling its 
vertices. $W$ is the image of $P ; U_{1}, \ldots, U_{m}$ are, in order, the distinct images of those $X_{i}$ such that $L X_{i}$ (in $\mathrm{K}$ ) $\geqslant \pi$; and $V_{1}, \ldots, V_{n}$ are defined similarly. I have hitherto assumed $\operatorname{im} \alpha$ and $\operatorname{im} \beta$ have no common initial portion, but the general case presents no further difficulty. This completes the proof of Lemma 3.6.

The next two lemmas enable one to generalize from Case II situations to Case III ones.

LEMMA 3.7. Let $\mathbf{M}$ be a $\mathbf{p}$-spherical metric complex, not necessarily having unique minimal paths. Let $\alpha$ be a geodesic. Then there is an $\epsilon \geqslant 0$ such that whenever $\beta$ is a path pointwise closer than $\epsilon$ to $\alpha$, there is a chain $B$ satisfying:

(1) $\alpha$ is within $B$;

(2) $\beta$ is a path in $B$; more strictly, $\beta$ can be lifted to a path in the development of $B$.

Proof. For each $t \in I$ let $\langle\alpha(t)\rangle$ be the simplex such that $\alpha(t) \in$ int $\langle\alpha(t)\rangle$. There is a minimal subdivision $0=t_{0} \leqslant t_{1} \leqslant \cdots \leqslant t_{k}=1$ of I such that $\langle\alpha(t)\rangle$ is the same simplex $a_{i}$ for all $t \in\left(t_{i-1}, t_{i}\right), i=1, \ldots, k$. Set $b_{i}=\left\langle\alpha\left(t_{i}\right)\right\rangle$; so $b_{i}=a_{i} \cap a_{i+1}$. Then $C=\left(a_{1}, \ldots, a_{k}\right)$ is a chain. Set $X_{0}=P, X_{k}=Q, X_{i}=$ $\operatorname{im} \alpha \cap b_{i}$ for $i=1, \ldots, k-1$, and say that $X_{i}=\alpha\left(u_{i}\right)$. Choose numbers $v_{0}=0, v_{k+1}=1$, and $v_{i}$ for $i=1, \ldots, k$ so that $u_{i-1} \leqslant v_{i} \leqslant u_{i}$, and set $Y_{i}=\alpha\left(v_{i}\right)$, for $i=1, \ldots, k$. Note that $Y_{i} \in$ int $a_{i}$ and $X_{i} \in$ int $b_{i}$ for $i=1$, $\ldots, k-1$. Choose $\epsilon$ so small that for each $i=0, \ldots, k$ the set $N_{i}=$ $\bigcup\left\{B_{\alpha(t)}(\epsilon, \mathbf{M})\right.$ for $\left.t \in\left[v_{i}, v_{i+1}\right]\right\}$ is contained in int $\operatorname{star}\left(X_{i}, \mathbf{M}\right)$. (See Diagram 7.)

Let $\beta$ be a path from $P$ to $Q$, pointwise closer than $\epsilon$ to $\alpha$. Let $B=$ $\left(c_{1}, \ldots, c_{n}\right)$ be the chain analogous to $C$ defined for $\beta$. Then $\beta$ is a path in $B$ (though not within $B$ ) in the sense of assertion (2). I have to show $\alpha$ is within $B$. Now im $\beta \subseteq \bigcup\left\{N_{i}\right\}=\bigcup\left\{\right.$ int $\left.\operatorname{star}\left(b_{i}, M\right)\right\}$. Hence every $\langle\beta(t)\rangle$ has at least one $b_{i}$ as face. For each $i=1, \ldots, k-2$ define the set $T_{i}$ to be $\{t \in$ $\left[v_{i}, v_{i+1}\right]$ such that $b_{t-1}$ is not a face of $\left.\langle\beta(t)\rangle\right\}$. To check that $T_{i} \neq \varnothing$, I now show that $v_{i+1} \in T_{i}$ : for $\beta\left(v_{i+1}\right)$ is within distance $\epsilon$ of $Y_{i+1}$, and hence $\beta\left(v_{i+1}\right)$ $\in \operatorname{int}\left(\operatorname{star}\left(X_{i}, \mathbf{M}\right) \cap \operatorname{star}\left(X_{i+1}, \mathbf{M}\right)\right)=$ int $\operatorname{star}\left(a_{i+1}, \mathbf{M}\right)$. So $a_{i+1}<\left\langle\beta\left(v_{i+1}\right)\right\rangle$. If $b_{i-1}$ were also $\left\langle\left\langle\beta\left(v_{i+1}\right)\right\rangle\right.$, this would say that $\alpha$ took two simplexes- $a_{i}$ and $a_{i+1}$-where one would do- $\left\langle\beta\left(v_{i+1}\right)\right\rangle$; in other words, $\alpha$ would not be a geodesic. Hence $v_{i+1} \in T_{i}$. Moreover $\beta\left(v_{i}\right) \in$ int $\operatorname{star}\left(X_{i-1}, \mathbf{M}\right)=$ int $\operatorname{star}\left(b_{i-1}, \mathbf{M}\right)$ again by choice of $\epsilon$, so $v_{i} \notin T_{i}$. It follows that g.l.b. $\left(T_{i}\right)$ is one of the $t_{j}$; call it $t_{j(i)}$. hence

Now $X_{i} \in d_{j(i)}=\left\langle\beta\left(t_{j(i)}\right)\right\rangle$. For $j(i) \leqslant j \leqslant j(i+1), v_{i-1} \leqslant t_{j} \leqslant v_{i+1} ;$

$$
\beta\left(t_{j}\right) \in \operatorname{int}\left(\operatorname{star}\left(X_{i}, M\right) \cup \operatorname{star}\left(X_{i+1}, M\right)\right)
$$




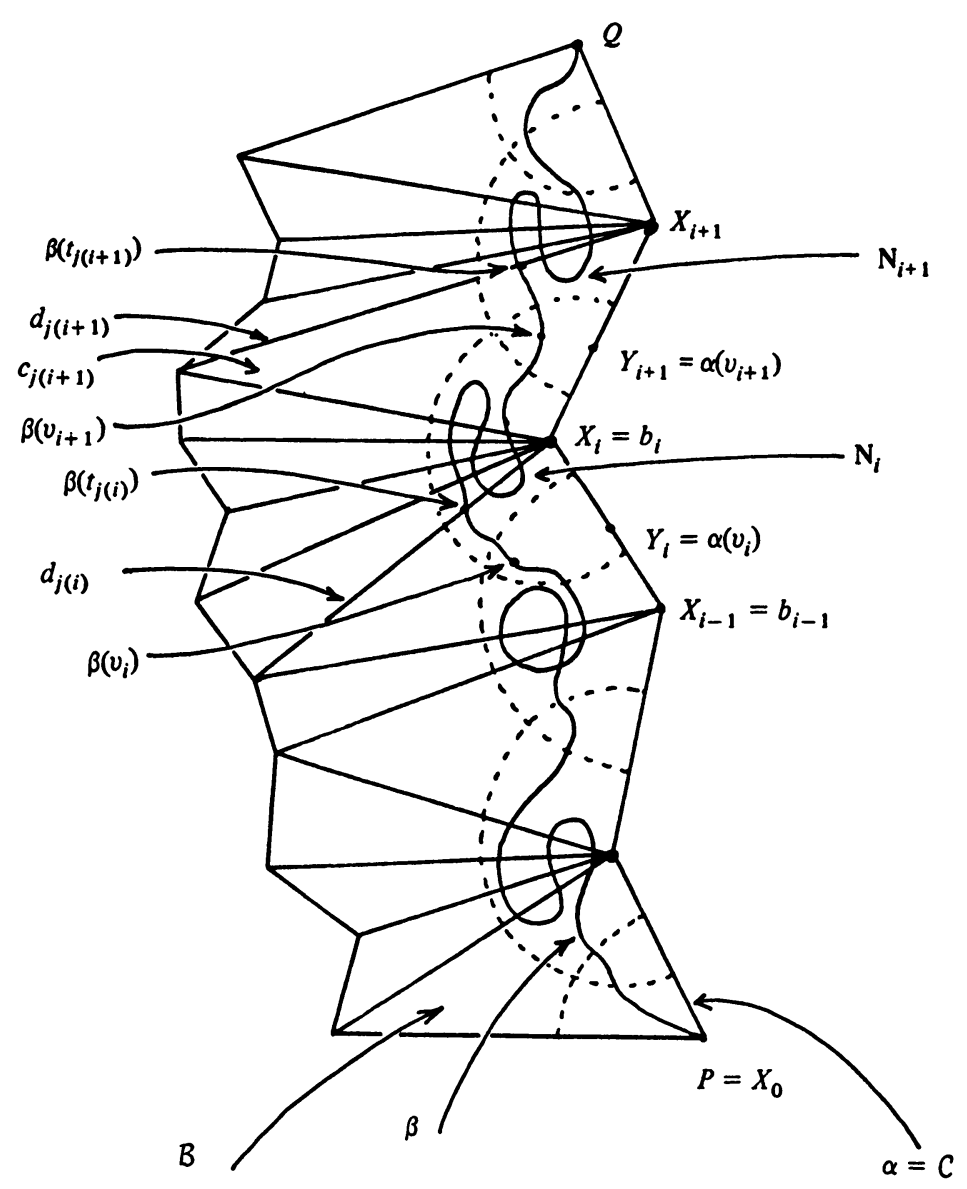

Diagram 7

By choice of $j(i+1), \beta\left(t_{j}\right) \in$ int $\operatorname{star}\left(X_{i}, \mathbf{M}\right)$. That is, $X_{i} \in d_{j}$ for $j(i) \leqslant j \leqslant$ $j(i+1)$. Any $c_{j}$ is spanned by $d_{j-1}$ and $d_{j}$, so $c_{j(i+1)}$ contains $X_{i}$ and $X_{i+1}$. Thus $\alpha$ is the path within $B$ determined by the points $Z_{j} \in d_{j}$ defined by $Z_{j}=X_{i}$ for $j(i) \leqslant j \leqslant j(i+1)$. This completes the proof of Lemma 3.7.

LemMa 3.8. Let $P, Q, R \in M$. Let $\gamma$ be a path from $Q$ to $R$, and for each $t$ let $\alpha_{t}$ be the minimal path from $P$ to $\gamma(t)$. Then there is a subdivision $0=t_{0}$ $\leqslant t_{1} \leqslant \cdots \leqslant t_{k}=1$ of I such that for each $i=0, \ldots, k-1, \alpha_{t_{i}}$ and $\alpha_{t_{i+1}}$ lie within the same chain.

Proof. Recall (Lemma 2.7) that $\alpha_{t}$ varies (pointwise) continuously in $t$. Let $T \subseteq I$ be the set of $t$ for which there exists a subdivision $0=t_{0} \leqslant \cdots \leqslant t_{j}$ $=t$ (which may vary with $t$ ) of $[0, t]$ such that $\alpha_{t_{i}}$ and $\alpha_{t_{i+1}}$ lie within the same chain. Applying Lemma 3.7 to $\alpha=\alpha_{0}$ and $\beta=\alpha_{t}$ as $t$ approaches 0 from above shows that $T \neq \varnothing$ (because in this case $\beta$, being a minimal path, is within 
the chain $B$ of that lemma). Let $t^{*}=1$.u.b. (T). Applying Lemma 3.7 as $t$ approaches $t^{*}$ from below shows that $t^{*} \in T$; applying the lemma once more as $t$ approaches $t^{*}$ from above would show there are $t \in T$ which are $\geqslant t^{*}$-unless $t^{*}=1$, which must therefore be the case. This proves Lemma 3.8.

Proposition 3.1 follows from:

LEMMA 3.9. Let $\mathbf{M}$ be a linear metric complex, $P \in \mathbf{M}, \alpha$ and $\beta$ minimal paths from $P$. Then $\rho(\alpha(t), \beta(t)) \leqslant t \rho(\alpha(1), \beta(1))$.

Proof of Proposition 3.1. Let $\gamma$ be the minimal path from $\alpha(0)$ to $\beta(1)$, and apply Lemma 3.9 first to $\alpha$ and $\gamma$, and then to $\gamma$ and $\beta$.

Proof of Lemma 3.9. The proof distinguishes three cases; most of the work is done in the first one.

Case I. $\alpha$ and $\beta$ are within the same chain $C$, and $Q$ and $R$ are in the same simplex. Let $K$ be the span of $\alpha$ and $\beta$. If $K$ degenerates to an arc, the required inequality is easily proved. Otherwise, $K$ is isometric to a complex $L \subseteq R^{2}$ of this form: $L=L_{1} \cup L_{2}$, where $L_{1}$ satisfies the hypotheses of Lemma 3.6. Extend the lines $U_{m-2}-U_{m-1}, \ldots, W-U_{1}, W-V_{1}, \ldots, V_{n-2}-V_{n-1}$ past their second endpoints till they meet $U_{m}-V_{n}$, say in $Y_{1}, \ldots, Y_{r}(r=m+n-2)$. Set $Y_{0}=Q, Y_{r+1}=R$, and let $\eta_{i}$ be the geodesic in $\mathrm{L}$ from $W^{\prime}$ to $Y_{i}$. It suffices to show that $\rho_{\mathrm{L}}\left(\eta_{i}(t), \eta_{i+1}(t)\right) \leqslant t \rho_{\mathrm{L}}\left(Y_{i}, Y_{i+1}\right)$, for $i=0, \ldots, r$. In other words the lemma is reduced to the case that $m=n=1$.

Now $\mathrm{L}=\langle U, V, W\rangle \cup\langle W, X\rangle$. Set $\alpha(t)=U^{\prime}$ and $\beta(t)=V^{\prime}$. If $U^{\prime}$ and $V^{\prime}$ both lie in $\alpha$ or in $\beta$, the required inequality is trivial, so I assume $U^{\prime} \in W-U$, $V^{\prime} \in W-V$. Taking polar coordinates at $W$, let $U-V$ have equation $r=r(\theta)$. Let $X-W$ have length $c$. Then $U^{\prime}-V^{\prime}$ is no longer than the smooth curve $\gamma$ from $U^{\prime}$ to $V^{\prime}$ defined by $r=r^{\prime}(\theta)=\operatorname{tr}(\theta)-(1-t) c$. Let $d s$ and $d s^{\prime}$ denote arc-length along $U-V$ and $\gamma$. Then $d r^{\prime}=t d r$; hence

$$
\left(d s^{\prime}\right)^{2}=\left(r^{\prime}\right)^{2}(d \theta)^{2}+\left(d r^{\prime}\right)^{2} \leqslant(t r)^{2}(d \theta)^{2}+(t d r)^{2}=t^{2}(d s)^{2} .
$$

So $d s^{\prime} \leqslant t d s$, and hence $L\left(U^{\prime}-V^{\prime}\right) \leqslant L(\gamma) \leqslant t L(U-V)$. The lemma is proved in Case I.

Case II. $\alpha$ and $\beta$ are within the same chain C. Say $Q \in a_{l}$ and $R \in a_{k}$ with $l \leqslant k$. Let the minimal path from $Q$ to $R$ be determined by $Z_{i} \in b_{i}$, for $i=l$, $\ldots, k-1$. Set $Z_{l-1}=Q, Z_{k}=R$, and let $\zeta_{i}$ be the geodesic from $P$ to $Z_{i}$, for $i=l-1, \ldots, k$. For each $i=l-1, \ldots, k-1, \rho_{c}\left(\zeta_{i}(t), \zeta_{i+1}(t)\right) \leqslant$ $t \rho_{C}\left(Z_{i}, Z_{i+1}\right)$ by Case I. Adding these inequalities for all $i$,

$$
\rho_{c}\left(\zeta_{l-1}(t), \zeta_{k}(t)\right) \leqslant \sum \rho_{c}\left(\zeta_{i}(t), \zeta_{i+1}(t)\right) \leqslant t \sum \rho_{c}\left(Z_{i}, Z_{i+1}\right)=t \rho(Q, R)
$$

which proves Case II.

Case III. $\alpha$ and $\beta$ general. Let $\gamma$ be the minimal path from $Q$ to $R$. By 
Lemma 3.8 there is a subdivision $0=t_{0} \leqslant t_{1} \leqslant \cdots \leqslant t_{k}=1$ of I such that for each $i=0, \ldots, k-1$ the minimal paths $\alpha_{i}$ and $\alpha_{i+1}$ from $P$ to $\gamma\left(t_{i}\right)$ and $\gamma\left(t_{i+1}\right)$ lie within some chain; of course $\alpha_{0}=\alpha$ and $\alpha_{k}=\beta$. I apply Case II to each consecutive pair of $\alpha_{i}$ 's and add the resulting inequalities; as in the proof of Case II this proves the lemma in general.

Lemmas 3.10 to 3.12 are the heart of the proofs of the other propositions. I shall prove first Lemma 3.10 in Case I, then part of Lemma 3.11, then Lemmas 3.10, 3.11 in full, and finally Lemma 3.12.

Lemma 3.10. Let $P, Q, R$ be such that $\rho(P, Q), \rho(P, R) \leqslant \pi \mathrm{p} / 2$. Assume there exists a geodesic $\gamma$ in $\mathbf{M}$ from $Q$ to $R$ which lies in int $B_{P}(\pi \mathrm{p} / 2, M)$. Then $\rho(P, \gamma(t)) \leqslant(1-t) \rho(P, Q)+t \rho(P, R)$ for all $t \in \mathrm{I}$, with equality only if $t=0$ or 1 , or if $P, Q$ and $R$ lie on a minimal path.

LEMMA 3.11. Let $\alpha$ be a minimal path from $Q$ to $P$ of length $\leqslant \pi \mathrm{p} / 2$. Let $\gamma:[0, \epsilon] \rightarrow M$ parametrize a short geodesic segment from $Q$ by arc-length. Then for $\epsilon$ sufficiently small (but nonzero):

(1) $\angle \bar{\alpha} Q \bar{\gamma} \leqslant \pi / 2 \Leftrightarrow \rho(P, \gamma(t))$ is strictly decreasing on some nondegenerate subinterval $[0, \delta]$;

(2) $L \bar{\alpha} Q \bar{\gamma} \geqslant \pi / 2 \Leftrightarrow \rho(P, \gamma(t))$ is strictly increasing on $[0, \epsilon]$.

Proof of Lemma 3.10, Case I. Assume the minimal paths $\alpha$ and $\beta$ from $P$ to $Q$ and $R$ lie in the same chain $C, Q$ and $R$ are in the same simplex, and $\gamma=Q-R$. I shall not assume $\gamma \subseteq$ int $B_{P}(\pi \mathrm{p} / 2, M)$ (this is implied by the inequality to be proved).

Let $\mathrm{K}$ be the span of $\alpha$ and $\beta$. By Lemma 3.6 $\mathrm{K}$ is isometric to a metric complex $\mathbf{L}$ in a p-spherical 2-sphere $S, L$ satisfying the assertions of that lemma. It suffices to prove the inequality with $\rho_{M}$ replaced by $\rho^{\prime \prime}=\rho_{L}$ and with $\mathbf{L}_{2}$ degenerate. Thus $I$ assume $L$ is a polygonal figure with vertices $W, U_{1}, \ldots, U_{m}$, $V_{n}, \ldots, V_{1}$; I have to show that if $Z$ divides $U_{m}-V_{n}$ in the ratio $1-t: t$, then $\rho^{\prime \prime}(W, Z) \leqslant(1-t) \rho^{\prime \prime}\left(W, U_{n}\right)+t \rho^{\prime \prime}\left(W, V_{n}\right)$. This is proved by induction on $m+n$. Let $w$ denote the length of $U_{m}-V_{n}, u_{1}, u_{2}, \ldots, u_{m}$ the lengths of $W-U_{1}, U_{1}-U_{2}, \ldots, U_{m-1}-U_{m}$, and let $u=u_{1}+u_{2}+\cdots+u_{m}=L(\alpha)$. Let $v$ be defined similarly. Let $z=\rho^{\prime \prime}(W, Z)$.

Iritial step. $m=n=1$. I first prove the inequality for the midpoint $Z_{0}$ of $U_{m}-V_{n}$. Let the rotation of $S$ through $\pi$ about the axis through $Z_{0}$ carry $W$ into $W^{\prime} ; U$ and $V$ are of course interchanged. Then $W-Z_{0}-W^{\prime}$ is a geodesic of length $2 z_{0}$. It is the unique minimal geodesic from $W$ to $W^{\prime}$ because $u, v \leqslant \pi \mathrm{p} / 2$ and $B_{W}(q, S)$ is convex whenever $q \leqslant \pi \mathrm{p} / 2$, so that $z_{0} \leqslant \pi \mathrm{p} / 2$. The path $W-U-$ $W^{\prime}$ has length $u+v$ and $U$ does not lie on $W-Z_{0}-W^{\prime}$ (or $\langle U, V, W\rangle$ would not be a 2 -simplex). Hence $2 z_{0} \leqslant u+v$, as required. 
By repeating this argument the strict inequality is shown whenever $t$ is a dyadic fraction, not 0 or 1 . Since $z$ varies continuously with $t$, the weak inequality is shown. But now any position of $Z$ other than $U, V$ or $Z_{0}$ lies between $Z_{0}$ and one of the others-say $U$. Then $Z$ divides $U-Z_{0}$ in the ratio $2(1-t): 2 t-1$. So $z \leqslant(2 t-1) u+2(1-t) z_{0} \leqslant(2 t-1) u+(1-t)(u+v)=t u+(1-t) v$; and the strict inequality is proved if $0 \leqslant t \leqslant 1 / 2$. A similar argument applies if $1 / 2 \leqslant t$ $\leqslant 1$; and the initial step of Case I of Lemma 3.10 is proved.

Inductive step. Now assume that $m$ (say) is $\geqslant 1$. L can be obtained from the convex triangle $\left\langle U_{m}, V_{n}, W\right\rangle$ by deleting the convex polygons $\mathrm{H}_{1}=W U_{1} U_{2}$ $\cdots U_{m}$ and $\mathbf{H}_{2}=W V_{1} V_{2} \cdots V_{n}$. Within $B_{W}(\pi \mathrm{p} / 2, \mathbf{S})$, if $W-U_{1}$ is extended beyond $U_{1}$ it cannot meet $\mathbf{H}_{1}$ after leaving $U_{1}$, nor can it meet $\mathrm{H}_{2}$ after leaving $W$. It follows that the extension of $W-U_{1}$ meets bdy $\mathrm{L}$ in just one point $Z_{0}$, which lies in $U_{m}-V_{n}$ and is not $U_{m}$ or $V_{n}$.

Let $W-Z_{0}$ have length $z_{0}$. Say $Z_{0}$ divides $U_{m}-V_{n}$ in the ratio $1-t_{0}: t_{0}$; then by the initial step since $t_{0} \neq 0$ or $1, z_{0} \leqslant t_{0} \rho_{S}\left(W, U_{m}\right)+\left(1-t_{0}\right) \rho_{S}\left(W, V_{n}\right)$ $\leqslant t_{0} u+\left(1-t_{0}\right) v$. Now $W-Z_{0}$ divides $L$ into two regions: $L^{\prime}$, with boundary $U_{1} \cdots U_{m} Z_{0}$, and $L^{\prime \prime}$, with boundary $W Z_{0} V_{n} \cdots V_{1}$. Both $L^{\prime}$ and $L^{\prime \prime}$ satisfy the hypotheses of this lemma and both have fewer vertices than $\mathbf{L}$, so by inductive hypothesis I may assume the lemma holds for $L^{\prime}$ and $L^{\prime \prime}$.

Let $Z$ divide $U_{m}-V_{n}$ in the ratio $1-t: t$ with $t \leqslant t_{0}$. Then $Z \in \mathrm{L}^{\prime \prime}$ and $\rho_{\mathrm{L}}(W, Z)=\rho_{\mathrm{L}}{ }^{\prime \prime}(W, X) . Z$ divides $Z_{0}-V_{n}$ in the ratio $1-\left(t / t_{0}\right): t / t_{0}$, so $z \leqslant$ $\left(t / t_{0}\right) z_{0}+\left(1-\left(t / t_{0}\right)\right) v \leqslant t u+(1-t) v$, with equality only if $t=0$.

If $t \geqslant t_{0}$, then $Z \in \mathrm{L}^{\prime}$ and divides $U_{m}-V_{n}$ in the ratio $(1-t) /\left(1-t_{0}\right)$ : $t /\left(1-t_{0}\right)$. Any minimal path from $Z$ to $W$ in $\mathbf{L}$ must lie in $L^{\prime}$ and consist of a minimal path from $Z$ to $U_{1}$ in $L^{\prime}$ followed by $U_{1}-W$. Hence $z \leqslant u_{1}+$ $\left(t /\left(1-t_{0}\right)\right)\left(u-u_{1}\right)+\left((1-t) /\left(1-t_{0}\right)\right)\left(z_{0}-u_{1}\right) \leqslant t u+(1-t) v$ again, with equality only if $t=1$. This completes the inductive proof of Case I of Lemma 3.10.

PART OF PROOF OF LEMMA 3.11. I now prove: If there are $t^{*}$ arbitrarily close to 0 such that $\rho\left(P, \gamma\left(t^{*}\right)\right) \leqslant L(\alpha)$, then $L \bar{\alpha} Q \bar{\gamma} \leqslant \pi / 2$.

Choose such a $t^{*}$ so small that $\alpha$ and the minimal path $\beta$ from $P$ to $R=$ $\gamma\left(t^{*}\right)$ lie within a common chain $C$. Let $\mathbf{K}$ be the span of $\alpha$ and $\beta$. I may assume im $\alpha$ and im $\beta$ have no common initial portion; in particular if $\mathbf{K}$ degenerates to an arc, then $\operatorname{im} \beta \subseteq \operatorname{im} \alpha$ and $L \bar{\alpha} Q \bar{\gamma}=0$. So $\mathbf{K}$ is isometric to a polygonal region $L_{1}$ satisfying the assertions of Lemma 3.6. If $t \geqslant 0$ is close enough to 0 , then the minimal path in $\mathbf{K}$ from $P$ to $\gamma(t)$ corresponds to the path $W-U_{1}-\cdots-U_{m-1}$ $-Z(t)$, where $Z(t)$ is the image of $\gamma(t)$. By Case I of Lemma 3.10 this path is strictly shorter than $\alpha$. Hence $L\left(U_{m-1}-Z(t)\right) \leqslant L\left(U_{m-1}-U_{m}\right)$, and this must be true for all small enough $t$. (**) of Lemma 2.2 shows that $\angle U_{m}$ of triangle $U_{m-1} U_{m} Z(t)$ must be $\leqslant \pi / 2$. That is, $L \bar{\alpha} Q \bar{\gamma}$ (in $\mathrm{K}$ ) $\leqslant \pi / 2$; a fortiori $L \bar{\alpha} Q \bar{\gamma}$ (in M) $\leqslant \pi / 2$. 
Proof of Lemma 3.10. By Lemma 3.8 there is a subdivision $0=t_{0} \leqslant$ $t_{1} \leqslant \cdots \leqslant t_{k}=1$ of I such that for each $i=0, \ldots, k-1$ the minimal paths from $P$ to $\gamma\left(t_{i}\right)$ and $\gamma\left(t_{i+1}\right)$ lie within a common chain. I use induction on $k$. The initial case, when $k=1$, is just Case I of this lemma.

Now say $k=2$. Set $Z=\gamma\left(t_{1}\right)$ and let $\zeta$ be the minimal path from $P$ to $Z$. Let $K^{\prime}$ be the span of $\alpha$ and $\zeta$ in some common chain, and $K^{\prime \prime}$ the span of $\zeta$ and $\beta$ in some chain. $K^{\prime}$ is isometric to $L^{\prime}=L_{1}^{\prime} \cup L_{2}^{\prime}$ satisfying the assertions of Lemma 3.6; so $L_{1}^{\prime}$ has vertices $W^{\prime} U_{1}^{\prime} \cdots U_{m}^{\prime}, V_{n^{\prime}}^{\prime} \cdots V_{1}^{\prime}$; and $L_{2}^{\prime}$ is a geodesic segment $W^{\prime}-X^{\prime}$, with $L_{1}^{\prime} \cap L_{2}^{\prime}=\left\{W^{\prime}\right\}$. Here $X^{\prime}-W^{\prime}-U_{1}^{\prime}-\cdots-U_{m}^{\prime}$, corresponds to $\alpha, X^{\prime}-W^{\prime}-V_{1}^{\prime}-\cdots-V_{n^{\prime}}^{\prime}$ to $\zeta$, and $X^{\prime}-W^{\prime}$ to whatever initial portion $\alpha$ and $\zeta$ have in common; of course $X^{\prime}$ may equal $W^{\prime}$. Similarly $\mathbf{K}^{\prime \prime}$ is isometric to a complex $\mathbf{L}^{\prime \prime} \subseteq S$, with $\mathbf{L}^{\prime \prime}=\mathbf{L}_{1}^{\prime \prime} \cup \mathbf{L}_{2}^{\prime \prime}$, where $\mathbf{L}_{1}^{\prime \prime}$ has vertices $W^{\prime \prime} U_{1}^{\prime \prime} \cdots U_{m}^{\prime \prime} V_{n}^{\prime \prime} \cdots V_{1}^{\prime \prime}$, and $L_{2}^{\prime \prime}=W^{\prime \prime}-X^{\prime \prime}$, such that $X^{\prime \prime}-W^{\prime \prime}-U_{1}^{\prime \prime}-\cdots-$ $U_{m}^{\prime \prime}$ corresponds to $\zeta$ and $X^{\prime \prime}-W^{\prime \prime}-V_{1}^{\prime \prime}-\cdots-V_{n}^{\prime \prime}$ to $\beta$. (See Diagram 8.) Set $u=L(\alpha), v=L(\beta), h=L(\gamma)$ and $z=L(\zeta)$. Say $Z=\gamma(\mu)$, so that $U_{m^{\prime}}^{\prime}-V_{n^{\prime}}^{\prime}$ has length $\mu h$ and $U_{m}^{\prime \prime}-V_{n}^{\prime \prime}$ length $\lambda h$, where $\lambda=1-\mu$. By Case I of this lemma it suffices-in this case, $k=2$-to show that $z \leqslant \lambda u+\mu v$, with equality only under the given circumstances.

If either $\mathbf{K}^{\prime}$ or $\mathbf{K}^{\prime \prime}$ degenerates into an arc, the required inequality follows from Case I of Lemma 3.10. So I now assume neither is degenerate. Let $Z_{t}^{\prime}$ and $Z_{t}^{\prime \prime}$ divide $V_{n^{\prime}}^{\prime}-U_{m^{\prime}}^{\prime}$ and $U_{m}^{\prime \prime}{ }^{\prime \prime} V_{n^{\prime \prime}}^{\prime \prime}$ in the ratio $t: 1-t$. For $t$ sufficiently small, the minimal path from $X^{\prime}$ to $Z_{t}^{\prime}$ in $L^{\prime}$ consists of the segments $X^{\prime}-W^{\prime}-$ $V_{1}^{\prime}-\cdots-V_{n^{\prime}-1}^{\prime}-Z_{t}^{\prime}$, and the minimal path from $X^{\prime \prime}$ to $Z_{t}^{\prime \prime}$ in $L^{\prime \prime}$ consists of the segments $X^{\prime \prime}-W^{\prime \prime}-U_{1}^{\prime \prime}-\cdots-U_{m}^{\prime \prime}-1-Z_{t}^{\prime \prime}$. Say these paths have lengths $u_{t}$ and $v_{t}$. An elementary calculation shows that it suffices to prove $z \leqslant \lambda u_{t}+\mu v_{t}$. Now up to first order in $t$,

$$
\begin{aligned}
& z=u_{t}+t \mu h \cos \left(L V_{n^{\prime}}^{\prime}\right)+O\left(t^{2}\right), \\
& z=v_{t}+t \lambda h \cos \left(L U_{m^{\prime \prime}}^{\prime \prime}\right)+O\left(t^{2}\right) .
\end{aligned}
$$

Since $\lambda+\mu=1, \lambda(1)+\mu(2)$ gives

$$
\begin{aligned}
z & =\lambda u_{t}+\mu v_{t}+\lambda \mu t h\left[\cos \left(L V_{n^{\prime}}^{\prime}\right)+\cos \left(L U_{m^{\prime \prime}}^{\prime \prime}\right)\right]+O\left(t^{2}\right) \\
& =\lambda u_{t}+\mu v_{t}+2 \lambda \mu t h \cos 1 / 2\left(L V_{n^{\prime}}^{\prime}+L U_{m^{\prime \prime}}^{\prime \prime}\right) \cos 1 / 2\left(L V_{n^{\prime}}^{\prime}-L U_{m^{\prime \prime}}^{\prime \prime}\right)+O\left(t^{2}\right) .
\end{aligned}
$$

The point is that since $\gamma$ is a geodesic, $L V_{n}^{\prime}+\angle U_{m}^{\prime \prime} \geqslant \pi$, so that the third term on the right-hand side is $\leqslant 0$. Thus, for $t$ small enough, $z \leqslant \lambda u_{t}+\mu v_{t}$ unless $\angle V_{n^{\prime}}^{\prime}+\angle U_{m^{\prime \prime}}^{\prime \prime}=\pi$. In this case, consider the figure formed by joining the 2 simplexes $\left\langle Z_{t}^{\prime}, V_{n^{\prime}-1}^{\prime}, V_{n^{\prime}}^{\prime}\right\rangle$ and $\left\langle Z_{t}^{\prime \prime}, U_{m^{\prime \prime}-1}^{n}, U_{m^{\prime \prime}}^{\prime \prime}\right\rangle$ along their common portion of $\zeta$. The argument used in Case I applies to this figure, and the desired inequality follows. 

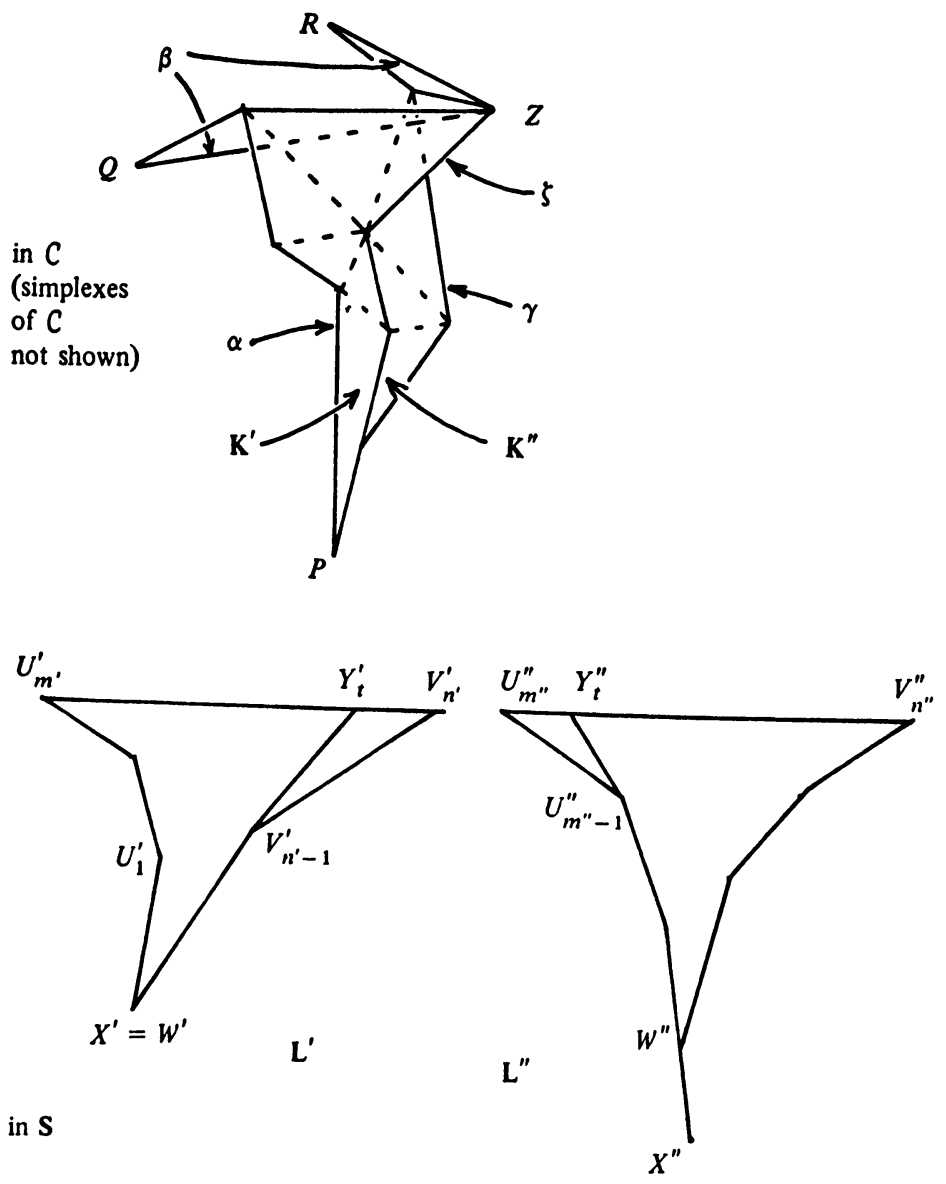

DiAgram 8

In general there is more than one $Z_{i}$ between $Q$ and $R$. Let $Z_{i}$ and $Z_{j}$ be the last distinct ones before $R$. By inductive hypothesis one can write down an inequality for $\rho_{C}\left(P, Z_{i}\right)$ in terms of $\rho_{C}(P, Q)$ and $\rho_{C}\left(P, Z_{j}\right)$. By the argument above one has an inequality for $\rho_{C}\left(P, Z_{j}\right)$ in terms of $\rho_{C}\left(P, Z_{i}\right)$ and $\rho_{C}(P, R)$. Combining these inequalities yields the desired result for $Z_{j}$. By inductive hypothesis the desired inequality holds for all the $Z$ 's, and hence also for points between them, by Case I. This completes the proof of Lemma 3.10.

Proof of Lemma 3.11. Choose $\epsilon \leqslant 1 / 2 \rho(Q, \operatorname{link}(Q, M)$ ) (cf. Lemma 2.2); then $\gamma$ and $\alpha \uparrow[0, \epsilon]$ are straight-line segments. It follows from Lemma 3.10 that there is a unique $\delta \in[0, \epsilon]$ at which $\rho(P, \gamma(t))$ takes on its minimum value; also that $\rho(P, \gamma(t))$ is strictly increasing on $[0, \epsilon] \Leftrightarrow \delta=0$, and strictly decreasing on $[0, \delta]$ if $\delta \neq 0$. So it suffices to show that $\delta \neq 0 \Leftrightarrow L \bar{\alpha} Q \bar{\gamma} \leqslant \pi / 2$.

The part of the proof of this lemma already given shows that if $\delta \neq 0$, then $\angle \bar{\alpha} Q \bar{\beta} \leqslant \pi / 2$. Now assume that $\angle \bar{\alpha} Q \bar{\beta} \leqslant \pi / 2$. Then for any $t \in[0, \epsilon]$ the 
minimal path $\beta_{t}$ from $\alpha(\epsilon)$ to $\gamma(t)$ has length given by (**) of Lemma 2.2:

$$
\cos \left(L\left(\beta_{t} / \mathbf{p}\right)\right)=\cos (\epsilon / \mathrm{p}) \cos (t / \mathbf{p})+\sin (\epsilon / \mathrm{p}) \sin (t / \mathbf{p}) \cos (L \bar{\alpha} Q \bar{\gamma}),
$$

if $p$ is finite, and

$$
\left(L\left(\beta_{t}\right)\right)^{2}=\epsilon^{2}+t^{2}-2 \epsilon t \cos (L \bar{\alpha} Q \bar{\gamma}), \text { if } p=\infty .
$$

In either case, $L\left(\beta_{t}\right) \leqslant \epsilon$ if $t$ is small enough. For such $t$,

$$
\rho(P, \gamma(t)) \leqslant \rho(P, \alpha(\epsilon))+\rho(\alpha(\epsilon), \gamma(t))=(L(\alpha)-\epsilon)+L\left(\beta_{t}\right) \leqslant L(\alpha) .
$$

Hence $\delta \neq 0$. This completes the proof of Lemma 3.11.

LeMma 3.12. Let $P, P^{\prime} \in \mathrm{M}$, let $q, q^{\prime} \leqslant \pi \mathrm{p} / 2$ and assume that $\rho\left(P, P^{\prime}\right) \leqslant$ $q+q^{\prime}$. Then $B_{P}(q, \mathbf{M}) \cap B_{P^{\prime}}\left(q^{\prime}, \mathbf{M}\right)$ is weakly convex in $\mathbf{M}$; in fact any minimal path in $\mathbf{B}_{P} \cap \mathbf{B}_{P^{\prime}}$ is a geodesic in $\mathbf{M}$.

Proof. Let $Q, R \in \mathrm{B}_{P} \cap \mathrm{B}_{P^{\prime}}$ and let $\gamma$ be a minimal path from $Q$ to $R$ in $\mathbf{B}_{P} \cap \mathbf{B}_{P^{\prime}}$; such a path exists because $\mathbf{B}_{P} \cap \mathbf{B}_{P^{\prime}}$ is compact. Since $\mathbf{B}_{P} \cap \mathbf{B}_{P^{\prime}}$ is not a metric complex, $\gamma$ need not $a$ priori be p.l.

Pick $\epsilon$ so that $0 \leqslant \epsilon \leqslant \min \left(\pi \mathrm{p} / 2-q, \pi \mathrm{p} / 2-q^{\prime}\right)$. Let $0=t_{0} \leqslant t_{1} \leqslant \cdots$ $\leqslant t_{k}=1$ be a subdivision of I such that $\rho_{M}\left(\gamma\left(t_{i}\right), \gamma\left(t_{i+1}\right)\right) \leqslant \epsilon$ for $i=0, \ldots$, $k-1$. Set $X_{i}=\gamma\left(t_{i}\right)$ for $i=0, \ldots, k$; so $X_{0}=Q, X_{k}=R$. Then the minimal path in $\mathbf{M}$ from $X_{i}$ to $X_{i+2}$ lies in int $B_{P}(\pi \mathrm{p} / 2, \mathrm{M}) \cap$ int $B_{P^{\prime}}(\pi \mathrm{p} / 2, \mathbf{M})$. By Lemma 3.10 this minimal path lies in $\mathbf{B}_{P} \cap \mathbf{B}_{P^{\prime}}$; hence $\gamma \uparrow\left[t_{i}, t_{i+1}\right]$ must be this minimal path. This shows that $\gamma$ is p.l. Moreover Lemma 3.10 shows that $\gamma\left(t_{i}, t_{i+2}\right) \subseteq$ int $\mathbf{B}_{P} \cap$ int $\mathbf{B}_{P^{\prime}}$. So $\gamma(0,1) \subseteq$ int $\mathbf{B}_{P} \cap$ int $\mathbf{B}_{P^{\prime}}$.

For each $t \neq 0$ or $1, T_{\gamma(t)}\left(\mathrm{B}_{P} \cap \mathrm{B}_{P^{\prime}}\right)=T_{\gamma(t)}(\mathrm{M})$. Thus the criteria for $\gamma$ to be a geodesic in $\mathbf{M}$ and in $\mathbf{B}_{P} \cap \mathbf{B}_{P^{\prime}}$ coincide. So $\gamma$ is a geodesic in $\mathbf{M}$ and the lemma is proved.

Proof of Proposition 3.2. Let $\alpha$ be a geodesic from $P$ to $Q$ and $\beta$ the minimal path from $P$ to $Q$, and assume that $L(\alpha) \leqslant \pi$ p. Suppose that $\alpha(1 / 2) \neq$ $\beta(1 / 2)$. Pick $q$ so that $L(\alpha) \leqslant 2 q \leqslant \pi$. Let $\gamma$ be a minimal path from $\alpha(1 / 2)$ to $\beta(1 / 2)$ in $B_{P}(q, \mathbf{M}) \cap B_{Q}(q, \mathbf{M})$. Then $\gamma$ is a geodesic in $\mathbf{M}$ by Lemma 3.12. Since $L(\alpha) \geqslant L(\beta)$ it follows from Lemma 3.10 that $\rho(P, \gamma(t))$ is decreasing in $t$ for $t$ sufficiently close to 0 . By the first part of the proof of Lemma $3.11, \angle \bar{\gamma} \alpha(1 / 2) \bar{\alpha}$

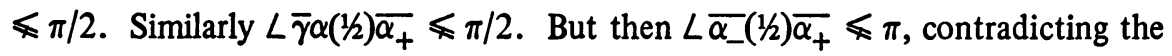
hypothesis that $\alpha$ is a geodesic. So $\alpha(1 / 2)=\beta(1 / 2)$. The same argument shows that $\alpha(1 / 4)=\beta(1 / 4)$ and so on, till $\alpha(t)=\beta(t)$ whenever $t$ is a dyadic fraction. By continuity $\alpha(t)=\beta(t)$ for all $t$, which proves the proposition.

Proof of Proposition 3.3. In view of Lemma 3.10 it suffices to prove that the minimal path $\gamma$ from $Q$ to $R$ lies in int $B_{P}(\pi \mathrm{p} / 2, \mathrm{M})$. Pick $q$ so that $\rho(P, Q), \rho(P, R) \leqslant q \leqslant \pi \mathrm{p} / 2$. Let $\gamma^{\prime \prime}$ be a minimal path in $B_{P}(q, \mathrm{M})$ from $Q$ to $R$. By Lemma 3.12, $\gamma^{\prime \prime}$ is a geodesic in M. Since $L\left(\gamma^{\prime \prime}\right) \leqslant 2 q \leqslant \pi$ p, $\gamma^{\prime \prime}=\gamma$ by 
Proposition 3.2. This proves Proposition 3.3.

Proof of Proposition 3.4. By Lemma $3.7 \epsilon$ can be chosen so that whenever $\beta$ is a path from $P$ to $Q$ pointwise closer than $\epsilon$ to $\alpha$, there is a chain $B$ such that $\beta$ is a path in $B$ and $\alpha$ is within $B$. Assume that $L(\beta) \leqslant L(\alpha)$; I have to show that $\operatorname{im} \beta=\operatorname{im} \alpha$. The proof is a modification of the proof of Proposition 3.2: one replaces $M$ by $B$ and uses Case I of Lemma 3.10 instead of the full version.

Proof of Proposition 3.5. It follows from Lemma 3.10 that $B_{P}(q, M)$ $\cap a$ is either empty, a single point or a strictly convex body with nonempty interior in $a$. Lemma 3.12 shows that at each point of (bdy $\left.B_{P}\right) \cap a$ there is a unique supporting hyperplane in $a$; and it follows that $B_{P} \cap a$ is smooth.

4. Negative curvature. In this section I shall prove:

THEOREM 1. Let $\mathbf{M}$ be a complete, simply-connected linear metric complex which is an n-manifold without boundary. Assume that $\kappa_{+}(P \bar{X}) \leqslant 0$ for all $P \in \mathbf{M}$ and all $P \bar{X} \subseteq \nu_{P}(\mathbf{M})$. Then $\mathbf{M}$ is p.l. isomorphic to $\mathbf{R}^{n}$.

For smooth manifolds the proof of the Cartan-Hadamard theorem falls into two parts (see J. Milnor [6]). First one shows, using the calculus of variations, that if $\mathbf{V}$ is complete, simply-connected and has everywhere nonpositive sectional curvature, then $\mathrm{V}$ has globally unique geodesics. It then follows that at any $P \in$ $\mathrm{V}$, the map $\exp _{P}: T_{P}(\mathrm{~V}) \rightarrow \mathrm{V}$ is everywhere nonsingular and is therefore a diffeomorphism. I shall follow this plan as best I can. Let me deal with the second part of the proof first, where I shall have to modify most the argument outlined above. This part of the proof will take us up to Corollary 4.6.

As Diagram 3 of $\$ 2$ shows, it is not at all clear how to define a global map " $\exp _{P}$ ": $T_{P}(\mathrm{M}) \rightarrow \mathrm{M}$, far less how to show it a homeomorphism. I shall replace the proof used in the smooth case by a "critical point" argument. Let $\rho: \mathbf{M} \rightarrow$ $\mathbf{R}$ be defined by $\rho(X)=\rho_{M}(P, X)$. The main step is to show that the only "critical point" of $\rho$ on $\mathbf{M}$ is $P$. This requires induction on $n$, the dimension of M.

For any $a \in M, \rho \uparrow a$ takes on its minimum value at just one point $\hat{a} \in a$ (Proposition 3.5). Starring $\mathbf{M}$ at all the $a$, in order of decreasing dimension, gives a subdivision, which I call $\mathbf{M}$ again, such that if $v$ and $w$ are vertices which span a 1 -simplex, then $\rho$ is strictly monotone on $v-w$. Lemma 4.4 will show that the vertices are the only possible "critical points" of $\rho$, and Lemma 4.5 , which makes use of the inductive hypothesis, will show that the vertices are not in fact "critical points" either.

I must first make a detour to discuss p.d. regular neighbourhoods. I do not know whether a general theory has been established for them, so I shall give an account which will suffice for the purposes of this paper.

Let $f: \mathbf{M} \rightarrow \mathbf{R}$ be a function such that for each $a \in \mathbf{M}, f \uparrow a$ is strictly 
convex, has smooth level surfaces and takes its minimum value at a vertex of $a$. In the paragraph before last, $\rho$ is a function of this type, once $M$ has been subdivided. Let $c$ be a number such that bdy $f^{c}$ contains no vertices of $M$. (Recall that $f^{c}$ is, by definition, $\{X \in M$ such that $f(X) \leqslant c\}$.) Define the subcomplex $\mathbf{K} \subset \mathbf{M}$ by $\mathbf{K}=\{a$ such that $f(v) \leqslant c$ for all vertices $v<a\}$. Then $\mathbf{K}$ is a full subcomplex of $\mathbf{M}$. If $b \in \mathbf{M}$ meets $\mathbf{K}$ but is not in $\mathbf{K}$, then $b$ can be uniquely expressed as $a^{\prime} * b^{\prime}$, where $a^{\prime} \in \mathbf{K}, b^{\prime} \cap \mathbf{K}=\varnothing$. $f^{c} \cap b$ is a smooth, strictly convex neighbourhood of $a^{\prime}$ in $b$ which does not meet $b^{\prime}$. Hence $f^{c}$ is a p.d. regular neighbourhood of $\mathbf{K}$ in $\mathbf{M}$; it is the only type of p.d. regular neighbourhood which I shall use in this paper. For example, let $\mathbf{M}$ be a p-spherical metric complex which satisfies the condition of $\S 3$ that whenever $Q, R \in M$ with $\rho(Q, R)$ $\leqslant \pi \mathrm{p}$, then there is a unique minimal path from $Q$ to $R$. Fix $P \in \mathrm{M}$, and let $\rho: \mathbf{M} \rightarrow \mathbf{R}$ denote the function distance-from $-P$. Then $\rho^{c}$ is a p.d. regular neighbourhood of some subcomplex of $\mathbf{M}$, as above, provided that $c \leqslant \pi \mathrm{p} / 2$, by Proposition 3.5. This type of example lends itself to inductive arguments; for if $\mathrm{M}$ is such a metric complex, let $r \leqslant 1 / 2 \rho(P, \operatorname{link}(P, \mathrm{M}))$, let $P^{*} \in S_{P}(r, \mathrm{M})$ and let $\rho^{*}: S_{P} \rightarrow \mathbf{R}$ denote the function distance-from- $P^{*}$ (in $S_{P}$ ); then $\rho^{* c}$ is also a p.d. regular neighbourhood in $S_{P}$, provided $c \leqslant \pi \mathrm{p} \sin (r / \mathrm{p}) / 2$, by Lemma 2.6 and Proposition 3.5.

To prove a uniqueness theorem for such regular neighbourhoods, the idea is to "linearize" $\mathbf{M}$ and $f$, so that the standard theorem for $\mathrm{pl}$. regular neighbourhoods can be applied (see for example Rourke and Sanderson [11]).

First let $\mathbf{M}$ be a $\mathrm{p}$-spherical metric complex, with $\mathrm{p}$ finite. I now construct a metrization $\mathbf{M}^{\prime \prime}$ of $\mathbf{M}$ as a linear metric complex, together with a p.d. isomorphism $\lambda: \mathbf{M} \rightarrow \mathbf{M}^{\prime \prime}$. The reverse of this construction-giving a linear metric complex a spherical metrization-will be used in $\S 5$. For each $a \in \mathbf{M}$, think of $a$ as a simplex in a p-sphere $S^{l} \subseteq \mathbf{R}^{l+1}$. Say $S$ has centre $C$ and let $a$ have vertices $v_{0}$, $\ldots, v_{k}$. Let $a^{\prime \prime}$ be the linear simplex $\left\langle v_{0}, \ldots, v_{k}\right\rangle \subseteq \mathbf{R}^{l+1}$. Since $C \notin[a]$, the affine space generated by $a$, radial projection from $C$ gives a diffeomorphism $\lambda_{a}: a \rightarrow a^{\prime \prime}$. Up to isometry, $a^{\prime \prime}$ and $\lambda_{a}$ depend only on $a$. In particular if $b<a$, then there is a natural isometry between $b^{\prime \prime}$ and $\lambda_{a}(b)<a^{\prime \prime}$ such that $\lambda_{b}$ corresponds to $\lambda_{a} \uparrow b$. Hence the linear metric complex $M^{\prime \prime}$ can be defined as $\left\{a^{\prime \prime}\right\}$ with incidence relations induced from those of $\mathbf{M}$, and the natural map $\lambda=\bigcup\left\{\lambda_{a}\right\}$ : $\mathbf{M} \rightarrow \mathbf{M}^{\prime \prime}$ is a simplicial isomorphism and is smooth on every simplex. $\lambda$ is called the linearization of $\mathbf{M}$.

For use in $\$ 5 I$ give the condition necessary for a linear metric complex $\mathbf{M}^{\prime \prime}$ to accept a p-spherical metrization. Let $a^{\prime \prime} \in \mathbf{M}^{\prime \prime}$ be a $k$-simplex, and think of $a^{\prime \prime}$ as a linear simplex in $\mathbf{R}^{l+1}$. Let the $(k-1)$-sphere circumscribed about $a^{\prime \prime}$ in [ $\left.a^{\prime \prime}\right]$ have radius $\mathrm{p}\left(a^{\prime \prime}\right)$. Let $\mathrm{S}^{l}$ be a sphere with radius $\mathrm{p}$ and centre $C$ circumscribing $a^{\prime \prime}$; then $\mathrm{p}$ must be $\geqslant \mathrm{p}\left(a^{\prime \prime}\right)$. If $\mathrm{p} \geqslant \mathrm{p}\left(a^{\prime \prime}\right)$, then $C \notin\left[a^{\prime \prime}\right]$, and radial projection 
from $C$ carries $a^{\prime \prime}$ onto a p-spherical $k$-simplex $a \subseteq \mathrm{S}$. If $\mathrm{p} \geqslant \mathrm{p}\left(a^{\prime \prime}\right)$ for all $a^{\prime \prime} \in \mathrm{M}^{\prime \prime}$, then following the previous construction I obtain a p-spherical metric complex $\mathbf{M}$ and a homeomorphism $\lambda^{\prime \prime}: \mathbf{M} \rightarrow \mathbf{M}^{\prime \prime}$ which is a simplicial isomorphism and is smooth on every simplex. $\lambda^{\prime \prime}$ will be called the p-spherical approximation to $\mathbf{M}^{\prime \prime}$. The second hypothesis of Theorem 3 is made to ensure that such a number $\mathbf{p}$ exists.

Let $\mathbf{M}$ be a $\mathbf{p}$-spherical metric complex, with $\mathbf{p}$ finite, and let $\lambda: M \rightarrow M^{\prime \prime}$ be its linearization. Set $f^{\prime \prime}=f \circ \lambda^{-1}$ ( $f$ as in the antepenultimate paragraph); then for each $a^{\prime \prime} \in \mathrm{M}^{\prime \prime}, f^{\prime \prime} \uparrow a^{\prime \prime}$ is strictly convex, has smooth level surfaces and takes its minimum value at a vertex of $a^{\prime \prime}$. So $f^{\prime \prime} c$ is a p.d. regular neighbourhood of $\mathbf{K}^{\prime \prime}=\lambda(\mathrm{K})$ in $\mathbf{M}^{\prime \prime}$. Define the linearization $f^{*}: M^{\prime \prime} \rightarrow \mathbf{R}$ of $f$ by $f^{*}(v)=f(v)$ for every vertex, and $f^{*}$ is linear on every simplex. Then $f^{* c}$ is a p.l. regular neighbourhood of $\mathrm{K}^{\prime \prime}$ in $\mathbf{M}^{\prime \prime}$.

LEMMA 4.1. Let $\mathrm{M}$ be a linear metric complex, let $f^{c}$ be a p.d. regular neighbourhood of $\mathbf{K}$, and let $f^{* c}$ be the linearization of $f^{c}$. Then there is a p.d. isomorphism $h: \mathbf{M} \rightarrow \mathbf{M}$ such that:

$h\left(f^{c}\right)=f^{* c}$;

$h(a)=a$ for every $a \in \mathbf{M}$, and $h \uparrow a$ is smooth;

$h \uparrow a$ is the identity if $a \in \mathbf{K}$ or if $a \cap \mathbf{K}=\varnothing$.

Proof. Let $\mathbf{N}=\bigcup$ int $b$ such that $b \cap \mathbf{K} \neq \varnothing$ but $b \notin \mathbf{K}$ \}. Then bdy $f^{* c}$ $\subseteq \mathrm{N}$. Let $X \in$ bdy $f^{* c}$; say $X \in \operatorname{int} b$, and write $b$ as $a^{\prime} * b^{\prime}$, with $a^{\prime} \in \mathrm{K}$ and $\bar{b}^{\prime} \cap \mathbf{K}=\varnothing$. There are unique points $Y_{X} \in a^{\prime}$ and $Z_{X} \in b^{\prime}$ such that $X \in Y_{X^{\prime}}-Z_{X}$; and then $Y_{X}-Z_{X} \cap$ bdy $f^{* c}=\{X\}$. As $X$ varies in bdy $f^{* c}$, the $\operatorname{int}\left(Y_{X^{-}}-Z_{X}\right)$ are disjoint and vary continuously, in fact smoothly in every simplex. Since $\mathbf{N}=$ $\bigcup\left\{\operatorname{int}\left(Y_{X}-Z_{X}\right)\right.$ for $X \in$ bdy $\left.f^{* c}\right\}$, it follows that $\mathrm{N}$ is a fibre bundle over bdy $f^{* c}$ with fibre $(0,1)$. The hypotheses on $f$ imply that bdy $f^{c}$ meets each fibre just once, and so can be regarded as a section $g$ of $\mathbf{N}$. It is now straightforward to define a p.d. isomorphism $h: \mathrm{cl} \mathbf{N} \rightarrow \mathrm{cl} \mathbf{N}$ such that:

$h$ preserves the fibres of $\mathbf{N}$;

$h\left(Y_{X^{-}}-X\right)=Y_{X^{-}}-g(X)$;

on $Y_{X}-Z_{X}, h$ is the identity near $Y_{X}$ and $Z_{X}$;

$h$ is smooth on every simplex.

Extend $h$ over all of $\mathbf{M}$ by the identity on those simplexes of $M$ which are contained in or disjoint from $\mathbf{K}$. Then $h$ satisfies the requirements of the lemma.

I now return to the proof of Theorem 1. In view of Lemma 4.1 it suffices to work with the linearization $\rho^{\prime \prime}$ of $\rho$ instead of with $\rho$.

PROPOSITION 4.2. Let $\mathrm{M}$ be a $\mathrm{p}$-spherical metric complex which is an $n$-manifold. Assume there are $P \in \mathrm{M}$ and a finite number $q \leqslant \pi \mathrm{p} / 2$ such that:

(1) $B_{P}(q, M)$ has a unique minimal paths and is disjoint from bdy $\mathbf{M}$; 
(2) for each $a \in M$ which meets $B_{P}(q, M), \rho \uparrow$ a takes on its minimum value at a vertex of $a$;

(3) $S_{P}(q, \mathbf{M})$ contains no vertices of $\mathbf{M}$. Then $\rho^{\prime \prime}$ is a p.l. n-disk.

Proof. The proof is by induction on $n$. It includes Lemmas 4.3 and 4.4, and Corollary 4.5 .

Consider $\left\{\rho(v)\right.$ for $v$ a vertex lying in $\left.B_{P}(q, \mathbf{M})\right\} \subseteq \mathbf{R}$, and write these numbers in strictly increasing order, say $d_{1}, \ldots, d_{k}$. Since $\mathbf{M}$ is complete and $q$ finite, there are only finitely many $d_{j}$.

LEMMA 4.3. If $d_{j} \leqslant c \leqslant e \leqslant d_{j+1}$, then there is a p.l. isomorphism $h: \mathbf{M}^{\prime \prime}$ $\rightarrow \mathrm{M}^{\prime \prime}$ such that:

$h\left(\rho^{\prime \prime}\right)=\rho^{\prime \prime}$;

$h(a)=a$ for every $a \in M^{\prime \prime}$;

$h \uparrow a$ is the identity if $a \subseteq \rho^{\prime \prime}$ or if $a \cap \rho^{\prime \prime c}=\varnothing$.

In other words, the vertices of $\mathrm{M}^{\prime \prime}$ are the only possible "critical points" of $\rho$ " up to level $q$.

Proof. Let $K^{\prime \prime}=\left\{a \in M^{\prime \prime}\right.$ such that $\rho^{\prime \prime}(v) \leqslant d_{j}$ for all vertices $\left.v<a\right\}$. Then $K^{\prime \prime}$ is a full subcomplex of $\mathbf{M}^{\prime \prime}$, and $\rho^{\prime \prime}$ and $\rho^{\prime \prime c}$ are both first-derived neighbourhoods of $K^{\prime \prime}$. The lemma is now a standard result; see for example Rourke and Sanderson $[11,3.6]$. The proof is analogous to that of Lemma 4.2, but not the same because the construction there is not p.l.

Lemma 4.4. Assume Proposition 4.2 in dimensions $\leqslant n-1$. Let $v$ be a vertex of $\mathbf{M}^{\prime \prime}$, and say $\rho^{\prime \prime}(v)=d_{j}$. Let $c$, e be numbers such that $d_{j-1} \leqslant c \leqslant d_{j}$ $\leqslant e \leqslant d_{j+1}$. Then there is a p.l. isomorphism $h: \operatorname{star}\left(v, \mathbf{M}^{\prime \prime}\right) \rightarrow \operatorname{star}\left(v, \mathbf{M}^{\prime \prime}\right)$ such that:

$h(a)=a$ for all $a \in \operatorname{link}\left(v, \mathbf{M}^{\prime \prime}\right)$;

$h \uparrow a$ is the identity if $a \in \operatorname{link}\left(v, \mathbf{M}^{\prime \prime}\right)$ is contained in or is disjoint from $\rho^{\prime \prime}$.

Proof. Set $\mathbf{N}^{e}=\rho^{\prime e} \cap \operatorname{star}\left(v, \mathbf{M}^{\prime \prime}\right), \mathbf{N}^{c}=\rho^{\prime c} \cap \operatorname{star}\left(v, \mathbf{M}^{\prime \prime}\right)$ and $\mathbf{L}^{c}=\rho^{\prime c}$ $\cap \operatorname{link}\left(v, \mathbf{M}^{\prime \prime}\right)$. On link(v, $\left.\mathbf{M}^{\prime \prime}\right), h$ can be constructed by Lemma 4.3. Let $\mathbf{K}=$ $\left\{a \in \operatorname{link}(v, \mathbf{M})\right.$ such that $\rho(w) \leqslant d_{j-1}$ for all vertices $\left.w<a\right\}$. Let $\lambda: \mathbf{M} \rightarrow \mathbf{M}^{\prime \prime}$ be the linearization of $M$, and let $K^{\prime \prime}=\lambda(K)$. Then $\mathbf{N}^{c}$ and $\mathbf{N}^{e}$ are first-derived neighbourhoods in $\operatorname{star}\left(v, \mathbf{M}^{\prime \prime}\right)$ of $\mathbf{K}^{\prime \prime}$ and $v * \mathbf{K}^{\prime \prime}$ respectively. I shall use the inductive hypothesis of Proposition 4.2 to show that $\mathrm{L}^{c}$ is an $(n-1)$-disk. An elementary argument in regular neighbourhood theory then shows that $\mathbf{N}^{c}$ and $\mathbf{N}^{e}$ are both $n$-disks which meet link $\left(v, \mathbf{M}^{\prime \prime}\right)$ in $(n-1)$-disks, and the desired extension of $h$ over $\operatorname{star}\left(v, \mathbf{M}^{\prime \prime}\right)$ can easily be made.

Recall that there is a natural simplicial isomorphism $g: \operatorname{link}(v, M) \rightarrow S_{v}(\mathbf{M})$. 
By virtue of the way in which $\mathbf{M}$ was subdivided, $g(\mathbf{K})$ can be characterized as $\left\{a \in S_{v}(\mathbf{M})\right.$ such that $\angle \bar{w} v \bar{\alpha} \leqslant \pi / 2$ for all vertices $\left.w<a\right\}$, where $\alpha$ is the minimal path from $v$ to $P$. Say $v \bar{\alpha}$ meets $S_{v}(\mathrm{M})$ in $P^{*}$, and let $\rho_{\mathrm{S}}$ denote the function distance-from. $P^{*}$ on $S_{v}(\mathrm{M})$. There is some number $r \leqslant \pi / 2$ such that $g(\mathbf{K}) \subseteq$ int $\rho_{\mathrm{S}}^{r}$. Then $\rho_{\mathrm{S}}^{r}$ is a p.d. regular neighbourhood of $g(\mathbf{K})$ in $S_{v}(\mathrm{M})$. By Lemma 2.6 and Proposition $3.3 \rho_{S}^{r}$ has unique minimal paths. Let $\lambda_{S}$ be the linearization of $S_{v}(\mathrm{M})$ and let $\rho^{*}$ be the linearization of $\rho_{\mathrm{S}}$. By inductive hypothesis $\rho^{* r}$ is a pd. (n-1)-disk. Applying the simplicial isomorphism $\lambda \circ g^{-1} \circ \lambda_{S}^{-1}$, this says that $\mathbf{K}^{\prime \prime}$ has a regular neighbourhood in $\operatorname{link}\left(v, \mathbf{M}^{\prime \prime}\right)$ which is a p.1. $(n-1)$ disk. Hence $\mathbf{L}^{c}$, which is another such regular neighbourhood, is also an $(n-1)$ disk, which is what remained to be proved.

Corollary 4.5. Assume Proposition 4.2 in dimensions $\leqslant n-1$. If $d_{j-1}$ $\leqslant c \leqslant d_{j} \leqslant e \leqslant d_{j+1}$, then there is a p.l. isomorphism $h: \mathbf{M} \rightarrow \mathbf{M}$ such that: $h\left(\rho^{\prime \prime e}\right)=\rho^{\prime \prime}$;

$h \uparrow a$ is the identity if $a \subseteq$ int $\rho^{\prime \prime c}$ or if $a \cap \rho^{\prime \prime} c=\varnothing$.

Proof. Let $v_{1}, \ldots, v_{l}$ be the vertices of $\mathbf{M}^{\prime \prime}$ such that $\rho^{\prime \prime}\left(v_{i}\right)=d_{j}$. Then the int $\operatorname{star}\left(v_{i}, \mathbf{M}^{\prime \prime}\right)$ are pairwise disjoint. Let $\mathbf{N}=\bigcup$ int $b$ such that $b \cap \rho^{\prime \prime} \neq \neq$ $\varnothing, b \nsubseteq \rho^{\prime \prime}$ and none of the $v_{i}$ is a vertex of $\left.b\right\}$. Define $h$ on $\mathrm{N}$ by the method of Lemma 4.3. In view of the second requirement on $h$, it remains only to define $h$ on $\bigcup\left\{\operatorname{star}\left(v_{i}, \mathbf{M}^{\prime \prime}\right)\right\}$; and Lemma 4.4 shows how to define $h$ on each $\operatorname{star}\left(v_{i}, \mathbf{M}^{\prime \prime}\right)$.

Proof of Proposition 4.2 In Dimension $n$, ASSUming its TRUTH IN DIMENSIONS $\leqslant n-1$. Choose numbers $c_{j}$, for $j=1, \ldots, k$ so that $c_{k}=q$ and $d_{j} \leqslant c_{j} \leqslant d_{j+1}$ for $j=1, \ldots, k-1$. Now $d_{1}=0$, since $P$ has become a vertex of $\mathbf{M}$. Hence $\rho^{\prime \prime} c_{1}$ is a convex neighbourhood of $P$ contained in $\operatorname{star}\left(P, M^{\prime \prime}\right)$ and so is a p.l. $n$-disk. By induction on $j$, using Corollary 4.5 at each step, it follows that $\rho^{\prime \prime q}$ is a pl. $n$-disk, which is what was to be proved.

COROLlary 4.6. Let $\mathrm{M}$ be a linear metric complex which is an n-manifold without boundary. Assume that $\mathrm{M}$ has unique geodesics to $P$. Then $\mathrm{M}$ is p.l. isomorphic to $\mathbf{R}^{n}$.

Proof. Assume M subdivided so that for every $a \in M, \rho \uparrow a$ takes its minimum value at a vertex of $a$. Let $d_{1}, \ldots, d_{k}, \ldots$ be defined as before. This time the $d_{k}$ form an infinite sequence, but since $M$ is complete, they have no cluster point in $\mathbf{R}$. Choose numbers $c_{k}$ as before, interpolated between the $d_{k}$; then $\lim c_{k}=\infty$. By Proposition 4.2, each $\rho^{\prime \prime} c_{k}$ is a pl. $n$-disk, and $\mathbf{M}=\bigcup\left\{\rho^{\prime \prime} c_{k}\right.$ for $k=1, \ldots$. By a theorem of J. Stallings [12] $\mathbf{M}$ is p.l. isomorphic to $\mathbf{R}^{n}$.

REMARK. By analysing p.d. regular neighbourhoods more thoroughly one could prove Lemma 4.4 even in case $d_{j}=e$. This would mean that the third 
hypothesis of Proposition 4.2 could be eliminated. As an addition to Theorem 1 one would have: for any $P \in \mathbf{M}$ and any finite number $q \geqslant 0, B_{P}(q, \mathbf{M})$ is a p.d. $n$-disk.

It remains to prove the first part of Theorem 1, which is implied by:

Proposition 4.7. Let $\mathrm{M}$ be a complete, connected linear metric complex such that $\kappa_{+}(P \bar{X}) \leqslant 0$ for all $P \in M$ and for all $P \bar{X} \subseteq \nu_{P}(M)$. Then for any $P, Q$ $\in \mathbf{M}$, there is just one geodesic in each homotopy class of continuous paths from $P$ to $Q$.

Proof. The proof will take up the rest of $\$ 4$. The method of proof is closely analogous to that used by Milnor [6] in the smooth case. Let $\Omega$ be the space of p.l. paths from $P$ to $Q$, with the $C^{0}$ topology. There is an energy function $E: \Omega \rightarrow \mathbf{R}$, defined in $\S 2$. I shall construct a family of finite-dimensional approximations $\Omega^{q}$ to $\Omega$. Analysing the "critical points" of $E \uparrow \Omega^{q}$ as in the proof of Proposition 4.2 will show that $\Omega^{q}$ can be deformed within itself onto a discrete set; the paths in $\mathbf{M}$ corresponding to points of this set are geodesics from $P$ to $Q$. Homotopy-theoretic considerations will show that as the $\Omega^{q}$ approximate $\Omega$, the corresponding discrete sets approximate the set of homotopy classes of paths from $P$ to $Q$.

Let $\alpha: \mathbf{I} \rightarrow \mathbf{M}$ be a path. Recall that for each $t \in \mathbf{I}, L(\alpha, t)$ denotes the length of $\alpha \uparrow[0, t]$, and the energy $E(\alpha)$ is defined to be $\int_{0}^{1}[d L(\alpha, t) / d t]^{2} d t$. It is easily checked that $E: \Omega \rightarrow R$ is continuous. Also $E(\alpha) \geqslant L(\alpha))^{2}$, and equality holds only when $\alpha$ is parametrized proportionally to arc-length. By Proposition 3.4, the local minima of $E$ are isolated points of $\Omega$ and represent exactly the geodesics in $\mathbf{M}$ from $P$ to $Q$. The main step in proving Proposition 4.7 is to show that $\Omega$ can be deformed onto the set of local minima of $E$.

Let $q$ be a positive number. Then $E^{q}=\{\alpha \in \Omega$ such that $E(\alpha) \leqslant q\}$ is contained in $B_{P}\left(q^{1 / 2}, M\right)$ which is compact. Hence there is a finite collection of balls $B_{X^{i}}\left(s_{i}, \mathbf{M}\right), i=1, \ldots, l$, such that: $\operatorname{im} \alpha \subseteq \bigcup\left\{\right.$ int $\left.B_{X^{i}}\left(s_{i}, M\right)\right\}$ for all $\alpha \in$ $E^{q} ; B_{X^{i}}\left(2 s_{i}, M\right) \subseteq \operatorname{star}\left(X^{i}, M\right)$, is strictly convex and has unique geodesics. Set $s=\min \left\{s_{i}\right\}$. There is an $\epsilon \geqslant 0$ such that $\epsilon \leqslant s$ and whenever $X, Y \in B_{P}(q, M)$ and $\rho(X, Y) \leqslant \epsilon$, then $X$ and $Y$ lie in some int $B_{X^{i}}\left(s_{i}, \mathbf{M}\right)$, and so there is a unique geodesic from $X$ to $Y$ in $M$. Set $\delta=\epsilon^{2} / q$. Whenever $\alpha \in E^{q}$ and $t, t^{\prime} \in I$ with $\left|t-t^{\prime}\right| \leqslant \delta$, then $\rho\left(\alpha(t), \alpha\left(t^{\prime}\right)\right) \leqslant \epsilon$. Choose a subdivision $0=t_{0} \leqslant t_{1} \leqslant \cdots \leqslant$ $t_{k+1}=1$ such that $t_{i+1}-t_{i} \leqslant \delta$, for $i=0, \ldots, k$. Define $\Omega^{q}\left(t_{1}, \ldots, t_{k}\right)=$ $\left\{\alpha \in E^{q}\right.$ such that $\alpha \uparrow\left[t_{i}, t_{i+1}\right]$ is a geodesic, for $\left.i=0, \ldots, k\right\}$; then $\Omega^{q}\left(t_{1}, \ldots, t_{k}\right)$, which I shall usually abbreviate to $\Omega^{q}$, is the type of approximation to $\Omega$ that will be used. I shall first prove that $\Omega^{q}$ can be deformed onto the set of local minima of $E \uparrow \Omega^{q}$, and then discuss how various of the $\Omega^{q}\left(t_{1}, \ldots, t_{k}\right)$ (for increasing $q$ and $k$ ) approximate $\Omega$. 
LEMMA 4.8. $\Omega^{q}\left(t_{1}, \ldots, t_{k}\right)$ is naturally homeomorphic to a compact subset of $\mathbf{M}^{k}$, the $k$-fold product of $\mathbf{M}$.

Proof. Define $f: \Omega^{q} \rightarrow \mathrm{M}^{k}$ by $f(\alpha)=\left(\alpha\left(t_{1}\right), \ldots, \alpha\left(t_{k}\right)\right)$; then $f$ is the required homeomorphism. The proof is quite similar to that of $[6,16.1]$. The only extra detail is to show that $f^{-1}$ is continuous. Let $\delta^{\prime} \leqslant \delta$ and let $\beta \in \Omega^{q}$ be such that $\rho\left(\beta\left(t_{i}\right), \alpha\left(t_{i}\right)\right) \leqslant \delta^{\prime}$ for $i=1, \ldots, k$. Then for each $i=0, \ldots$, $k+1, \alpha\left(t_{i}\right), \alpha\left(t_{i+1}\right), \beta\left(t_{i}\right)$ and $\beta\left(t_{i+1}\right)$ all lie in some $B_{X^{j}}\left(2 s_{j}, M\right)$. This ball has unique geodesics in $\mathbf{M}$, so by Proposition 3.1, $\rho(\beta(t), \alpha(t)) \leqslant\left(q \delta^{\prime}\right)^{1 / 2}$ for all $t \in$ $\left[t_{i}, t_{i+1}\right]$. Hence $\beta$ is pointwise closer then $\left(q \delta^{\prime}\right)^{1 / 2}$ to $\alpha$. Thus $f^{-1}: \operatorname{im} f \rightarrow \Omega^{q}$ is continuous, and the lemma is proved.

Henceforth I shall suppress $f$ and identify $\Omega^{q}$ with its image. In this context $E: \Omega^{q} \rightarrow \mathbf{R}$ can be defined thus: if $Z=\left(Z_{1}, \ldots, Z_{k}\right) \in \Omega^{q}$, then setting $Z_{0}=P, Z_{k+1}=Q$,

$$
E(Z)=\sum\left\{\left[\rho_{M}\left(Z_{i}, Z_{i+1}\right)\right]^{2} /\left(t_{i+1}-t_{i}\right) \text { for } i=0, \ldots, k\right\} .
$$

$E$ can be defined by this formula over all of $M^{k}$. For $d \leqslant q,\left(E \uparrow \Omega^{q}\right)^{d}=\{Z$ such that $E(Z) \leqslant d\}$ can be identified with $\Omega^{d}\left(t_{1}, \ldots, t_{k}\right)$; this I shall do.

Let $\mathrm{B} \subseteq \mathrm{M}^{k}$ be a $k$-fold product of independently chosen $B_{X^{i}}\left(s_{i}, \mathbf{M}\right)$. Index the various such sets $\mathbf{B}$ as $\mathbf{B}_{1}, \ldots, \mathbf{B}_{h}\left(\right.$ so $\left.h=l^{k}\right)$. Then $\Omega^{q} \subseteq \bigcup$ int $B_{j}$ \}, and by Lemma 2.4 each $\mathbf{B}_{j}$ is convex in $\mathbf{M}^{k}$ and has unique geodesics.

LEMmA 4.9. Let $\mathbf{B}$ be one of the $\mathbf{B}_{i}$. Then $E$ is strictly convex on $\mathbf{B}$.

Proof. Let $Y=\left(Y_{1}, \ldots, Y_{k}\right)$ and $Z=\left(Z_{1}, \ldots, Z_{k}\right)$ be in B. Set $Y_{0}=Z_{0}=P$ and $Y_{k+1}=Z_{k+1}=Q$. Then for each $i=0, \ldots, k+1, Y_{i}$ and $Z_{i}$ are both in some $B_{X^{j}}\left(s_{j}, M\right)$; hence for $i=0, \ldots, k, Y_{i}, Y_{i+1}, Z_{i}$ and $Z_{i+1}$ are all in $B_{X^{j}}\left(2 s_{j}, M\right)$, which is strictly convex and has unique geodesics in M.

Let $\beta$ be the geodesic in $\mathbf{M}^{k}$ from $Y$ to $Z$. Projecting $\beta$ into the $i$ th factor $M_{i}$ gives the geodesic $\beta_{i}$ from $Y_{i}$ to $Z_{i}$. By Propositions 3.1 and 3.3,

$$
\rho\left(P, \beta_{1}(t)\right) \leqslant(1-t) \rho\left(P, Y_{1}\right)+t \rho\left(P, Z_{1}\right) ;
$$

$$
\rho\left(\beta_{k}(t), Q\right) \leqslant(1-t) \rho\left(Y_{k}, Q\right)+t \rho\left(Z_{k}, Q\right) ;
$$

$$
\rho\left(\beta_{i}(t), \beta_{i+1}(t)\right) \leqslant(1-t) \rho\left(Y_{i}, Y_{i+1}\right)+t \rho\left(Z_{i}, Z_{i+1}\right) \text { for } i=1, \ldots, k-1 \text {. }
$$

The first two inequalities are strict unless $P, Y_{1}$ and $Z_{1}$, or $Q, Y_{k}$ and $Z_{k}$, lie on a geodesic. Now squaring an inequality among nonnegative numbers of the form $x \leqslant(1-t) y+t z$ gives $x^{2} \leqslant(1-t) y^{2}+t z^{2}$, with equality only if:

(1) the original inequality is an equality, and

(2) either $t=0$ or 1 , or $y=z$.

It follows from the inequalities $(*)$ that $E(\beta(t)) \leqslant(1-t) E(Y)+t E(Z)$, with equality only if $t=0$ or 1 , or if: 
(1) the paths in $M$ determined by $Y$ and $Z$ have the same image, and

(2) $\rho\left(Y_{i}, Y_{i+1}\right)=\rho\left(Z_{i}, Z_{i+1}\right)$ for $i=0, \ldots, k$.

These two conditions imply that $Y=Z$, which proves the lemma.

I now proceed, as in the proof of Proposition 4.2, to analyse the "critical points" of $E$ on $\Omega^{q}$. Let $\mathbf{M}^{*}$ be a subdivision of $\mathbf{M}^{k}$ such that:

(1) for every vertex $v \in \mathbf{M}^{*}$ which lies in $\Omega^{q}, \operatorname{star}\left(v, \mathbf{M}^{*}\right)$ is contained in some int $\mathbf{B}_{j}$;

(2) for every $a \in M^{*}$ which meets $\Omega^{q}, E \uparrow a$ takes on its minimum at a vertex of $a$.

Let $E^{*}: \mathbf{M}^{*} \rightarrow \mathbf{R}$ be the linearization of $E$ (recall that $\mathbf{M}^{*}$ is a linear metric complex). For every $a \in M^{*}$ which meets $\Omega^{q}, E \uparrow a$ is strictly convex. Hence $E^{* d} \subseteq E^{d}$ whenever $d \leqslant q$. $E^{*}$.

LEMMA 4.10. For any $d \leqslant q$ there is a deformation retraction of $\Omega^{d}$ onto

Proof. The proof is very close to that of Lemma 4.2, and I omit it.

Let $d_{1}, \ldots, d_{l}$ be the distinct elements, arranged in increasing order, of $\left\{E(v)\right.$ for $v \in \Omega^{q}$ and $v$ a vertex of $\left.\mathbf{M}^{*}\right\}$; since $\Omega^{q}$ is compact, there are only finitely many such $v$.

LEMMA 4.11. If $d_{j} \leqslant c \leqslant e \leqslant d_{j+1}$, then there is a deformation retraction $h_{t}$ of $E^{* e}$ onto $E^{* c}$ such that $h_{t}\left(a \cap E^{* e}\right) \subseteq$ a for every a which meets $E^{* e}$.

Proof. As in the proof of Lemma 4.3, $E^{* e}$ and $E^{* c}$ are both first-derived neighbourhoods of a subcomplex of $M^{*}$. Since $E^{* c} \subseteq$ int $E^{* e}$, the lemma follows from Rourke and Sanderson [11, 3.18].

LEMma 4.12. Let $d_{j-1} \leqslant c \leqslant d_{j} \leqslant e \leqslant d_{j+1}$, and let $v$ be a vertex of $\mathrm{M}^{*}$ such that $E(v)=d_{i}$. Assume that $E^{* c} \cap \operatorname{star}\left(v, \mathbf{M}^{*}\right) \neq \varnothing$. Then there is a deformation retraction of $E^{* c} \cup\left(E^{* e} \cap \operatorname{star}\left(v, M^{*}\right)\right)$ onto $E^{* c}$.

Proof. Let $\mathbf{N}^{c}=E^{* c} \cap \operatorname{star}\left(v, \mathbf{M}^{*}\right), \mathbf{L}^{c}=E^{* c} \cap \operatorname{link}\left(v, \mathbf{M}^{*}\right)$ and $\mathrm{J}^{c}=$ $\operatorname{cl}\left[\right.$ bdy $\left.\mathbf{N}^{c}-\mathbf{L}^{c}\right]$. Let $\mathbf{N}^{e}, \mathbf{L}^{e}$ and $\mathbf{J}^{e}$ be defined similarly. Set $\mathbf{H}=\operatorname{cl}\left[\mathbf{N}^{e}-\mathbf{N}^{c}\right]$ and $\mathbf{G}=\operatorname{cl}\left[\mathrm{L}^{e}-\mathrm{L}^{c}\right]$.

$\mathrm{H}$ is a cone from $v$ to $\mathrm{J}^{e} \cup \mathrm{G} \cup \mathrm{J}^{c}$, and so can be deformed onto $v *$ $\left(G \cup J^{c}\right)$. The proof of Lemma 4.11 gives a deformation retraction of $L^{e}$ onto $\mathbf{L}^{c}$. Hence $\mathbf{G} \cup \mathrm{J}^{c}$ has $\mathbf{J}^{c}$ as deformation retract, and so $v *\left(\mathbf{G} \cup \mathbf{J}^{c}\right)$ can be deformed onto $v * \mathrm{~J}^{c}$. Now $\operatorname{star}\left(v, \mathbf{M}^{*}\right)$ is contained in some convex $\mathbf{B} \subseteq \mathbf{M}^{*}$ in which geodesics are unique, and therefore vary continuously with their endpoints (Lemma 2.7). Pick a point $W \in E^{* c} \cap \mathrm{B}$ and let $\alpha$ be the geodesic from $v$ to $W$. For each $Z \in \mathrm{J}^{c}$ and each $t \in \mathrm{I}$, let $\beta_{Z}^{t}$ be the geodesic from $\alpha(t)$ to $Z$. Then $v * \mathrm{~J}^{c}=\bigcup\left\{\beta_{Z}^{0}\right.$ for $\left.Z \in \mathrm{J}^{c}\right\}$. Define a homotopy $f_{t}$ of $v * \mathrm{~J}^{c}$ by $f_{t}\left(\beta_{Z}^{0}(u)\right)=$ 
$\beta_{Z}^{t}(u)$. Since $\Omega^{c} \cap \mathrm{B}$ is convex, by Lemma $4.9, f_{1}\left(v * \mathrm{~J}^{c}\right) \subseteq \Omega^{c}$. By Lemma $4.10, v * \mathrm{~J}^{c}$ can be further deformed into $E^{* c}$. This completes the proof of Lemma 4.12.

Corollary 4.13. Let $d_{j-1} \leqslant c \leqslant d_{j} \leqslant e \leqslant d_{j+1}$. Then $E^{* e}$ has as deformation retract $E^{* c} \cup\left\{v \in M^{*}\right.$ such that $E(v)=d_{j}$ and $v$ is a local minimum of $E$.

Proof. The proof is similar to that of Corollary 4.5 .

COROLlARY 4.14. $\Omega^{q}$ has as deformation retract the discrete set of local minima of $E$. This discrete set can be identified with the set $\pi_{0}\left(\Omega^{c}\right)$ of components of $\Omega^{c}$.

The rest of the proof of Proposition 4.7 connects the topology of $\Omega^{q}$ to that of $M$. As in Milnor $[6,16.2]$ one can show that there is a deformation retraction of $E^{q}$ onto $\Omega^{q}$. The local minima of $E \uparrow E^{q}$ correspond to geodesics in $M$ from $P$ to $Q$ of length $\leqslant q^{1 / 2}$, and hence these local minima are in $\Omega^{q}$ all along. Thus each component of $E^{q}$ has exactly one local minimum of $E$. Hence whenever $q \leqslant r$, the inclusion $E^{q} \subseteq E^{r}$ induces a one-to-one map $\pi_{0}\left(E^{q}\right) \subseteq$ $\pi_{0}(E)$. Now $\Omega$ is the direct limit of $\left\{E^{q}\right.$ for $\left.q \in \mathbf{R}\right\}$; and it is easily seen that $\pi_{0}(\Omega)=\underset{\lim }{\longrightarrow} \pi_{0}\left(E^{q}\right)$. Hence there is exactly one geodesic from $P$ to $Q$ in each component of $\Omega$. But $\pi_{0}(\Omega)$ represents the set of homotopy classes of continuous paths from $P$ to $Q$, by the simplicial approximation theorem. This proves Proposition 4.7, and with it Theorem 1.

5. Positive curvature. The bulk of this section-up to Lemma 5.5-is devoted to proving Theorem 3; the techniques of its proof will give Theorem 2 as a by-product.

THEOREM 3. Let $\mathbf{M}$ be a complete, linear metric complex which is an nmanifold without boundary. Assume:

(1) there is a number $k \geqslant 0$ such that whenever $a$ is an $(n-2)$-simplex, $P$ $\in$ int $a$ and $P \bar{X} \subseteq \nu_{P}(\mathrm{M})$, then $\kappa_{-}(P \bar{X}) \geqslant \kappa$;

(2) there is a number $\mathrm{p}$ such that whenever $a$ is an $n$-simplex of $M$ and is represented as a linear simplex in $\mathbf{R}^{n}$, then the $(n-1)$-sphere circumscribed about a has radius $\leqslant \mathrm{p}$.

\section{Then}

(i) $\kappa_{-}(P \bar{X}) \geqslant 0$ for all $P \bar{X} \subseteq \nu_{P}(M)$, provided that $P$ is in the $(n-2)$-skeleton of $\mathbf{M}$;

(ii) $\mathbf{M}$ is compact; diam $\mathbf{M} \leqslant 2 \pi \mathrm{p}$ if $\kappa \geqslant\left(2-2^{1 / 2}\right) \pi$ and diam $\mathbf{M} \leqslant$ $\pi \mathrm{p} 4 \pi^{2} /\left[(2 \pi-\kappa)\left(4 \pi \kappa-\kappa^{2}\right)^{1 / 2}\right]$ otherwise.

The first hypothesis implies that the whole $(n-2)$-skeleton $\mathbf{M}^{n-2}$, as a 
simplicial complex, is intrinsic to $\mathbf{M}$, for it is the coarsest possible triangulation of the "singular set" of $\mathbf{M}$-that is, of the set of points where the normal geometry is nontrivial. The second hypothesis then says that the singular set is "fairly dense" in $\mathbf{M}$; it implies for example that every point of $\mathbf{M}$ is distant at most $\mathbf{p}$ from the singular set.

The proof relies heavily on the combinatorial structure $\mathrm{M}^{n-2}$ on the singular set. I think that some more general form of the theorem must be true in which no such combinatorial structure is required, but the general local shape and size of the singular set are more carefully restricted. I have already mentioned my belief that it should also be possible to weaken the curvature assumption if one has the right analogue for pl. manifolds of Ricci curvature in smooth ones.

The plan of the proof is this: I first prove an analogue of Theorem 3 for p-spherical metric complexes with $p$ finite, and show that such metric complexes have diameter $\leqslant \pi p$ (Proposition 5.3). A linear metric complex $M$ satisfying the second hypothesis of Theorem 3 has a $\mathbf{q}$-spherical approximation $\mathbf{M}^{*}$ once $\mathbf{q}$ is large enough. If $\mathbf{M}$ satisfies the first hypothesis of the theorem as well, then $\mathbf{M}^{*}$ satisfies the hypotheses of Proposition 5.3, if $q$ is large enough (Lemma 5.4). Hence $M^{*}$, and therefore $M$, is compact. It remains to compare $\rho_{M}$ to $\rho_{M}$ * (Lemma 5.5) and to estimate how small a value of $q$ will do.

Let $\mathbf{M}$ be a $\mathbf{p}$-spherical metric complex which is an $n$-manifold without boundary. The Curvature Hypothesis is this assumption on $\mathbf{M}$ : if $a \in \mathbf{M}$ with $\operatorname{dim} a \leqslant n-2$ and if $P \in$ int $a$, then $\operatorname{dim} \varphi_{P}(M)=\operatorname{dim} a$ and $\kappa_{-}(P \bar{X}) \geqslant 0$ for all $P \bar{X} \subseteq \nu_{P}(\mathrm{M})$. The Curvature Hypothesis for $(n-2)$-simplexes is the same, except with " $\operatorname{dim} a \leqslant n-2$ " replaced by "dim $a=n-2$ ". By Lemma 2.8 the the Curvature Hypothesis is equivalent to this condition: whenever $P \in$ int $a$ and $\operatorname{dim} a \leqslant n-2$, then $\varphi_{P}(\mathrm{M})=T_{P}(a)$ and $\operatorname{diam} \sigma_{P}(\mathrm{M}) \leqslant \pi$. This is the form in which the Curvature Hypothesis will be applied. The Curvature Hypothesis for $(n-2)$-simplexes can be expressed similarly.

Lemma 5.1. The Curvature Hypothesis is equivalent to the condition: if $\alpha$ is a geodesic from $P$ to $Q$ in $\mathbf{M}$, then $\alpha$ meets $\mathbf{M}^{n-2}$ at most in its endpoints, unless $P$ and $Q$ lie in the same simplex.

Proof. First assume the Curvature Hypothesis. Let $\alpha$ be a geodesic. For each $t \in(0,1)$ let $a(t)$ be the simplex such that $\alpha(t) \in$ int $a(t)$. Choose $t$ so that $\operatorname{dim} a(t)$ is as small as possible. It suffices to show that if $P$ and $Q$ do not lie in $a(t)$, then $\operatorname{dim} a(t)=n-1$. (If $\operatorname{dim} a(t)=n$, then $P$ and $Q$ have to be in $a(t)$.)

Suppose $\operatorname{dim} a(t) \leqslant n-2$. Set $R=\alpha(t), a=a(t)$. The tangent directions $R \overline{\alpha_{+}}$and $R \overline{\alpha_{-}}$cannot both be tangent to $a$, because then $\alpha \cap a$ would be a segment $X-Y$ with at least one of $X$ and $Y$ lying in bdy $a \cap$ int $\alpha$, which would contradict the choice of $t$. So say $R \overline{\alpha_{+}} \nsubseteq T_{R}(a)$. 
Now $T_{R}(\mathrm{M})=T_{R}(a) \times \nu_{R}(\mathrm{M})$ by the Curvature Hypothesis. Let $\mathrm{P}$ be the projection into the second factor. Then $P\left(R \overline{\alpha_{+}}\right)$is a genuine direction $R \overline{X^{\prime}} \subseteq$ $\nu_{R}(\mathrm{M}) . T_{R}(\alpha)$ defines a geodesic in $T_{R}(\mathrm{M})$ near $R$, by Lemma 2.5 ; hence $P\left(T_{R}(\alpha)\right)$ defines a geodesic in $\nu_{R}(\mathrm{M})$ near $R$, by Lemma 2.4. Thus $P\left(R \overline{\alpha_{-}}\right)$must be a genuine direction in $\nu_{R}(\mathrm{M})$ also; call it $R \bar{Y}^{\prime}$. By the Curvature Hypothesis again, $\angle \overline{X^{\prime}} R \overline{Y^{\prime}} \leqslant \pi$; so $P\left(T_{R}(\alpha)\right)$ is not a geodesic near $R$ after all. This contradiction establishes the first part of the lemma.

Conversely, assume geodesics avoid $\mathrm{M}^{n-2}$. Let $\operatorname{dim} a \leqslant n-2$ and let $P \in$ int $a$. By looking at geodesics in $\exp _{P}\left(\nu_{P}(\mathrm{M})\right)$ one can show that $\kappa_{-}(P \bar{X}) \geqslant 0$ for all $P \bar{X} \subseteq \nu_{P}(\mathrm{M})$. Clearly $\varphi_{P}(\mathrm{M}) \supseteq T_{P}(a)$. Suppose they are not equal. Then there is a geodesic $\alpha^{\prime}$ through $P$ in $\varphi_{P}(M)$ which does not lie in $T_{P}(a)$. But $\exp _{P}\left(\alpha^{\prime}\right)$ is then a geodesic through $P$ in $\mathbf{M}$ which cannot lie in a single simplex. This is contrary to the assumption, so $\varphi_{P}(\mathrm{M})=T_{P}(a)$. This completes the proof of Lemma 5.1.

LEMMA 5.2. Let $\mathrm{M}$ be a $\mathrm{p}$-spherical metric complex with $\mathrm{p}$ finite, which is an n-manifold without boundary. Assume the Curvature Hypothesis holds for $\mathbf{M}$. Then $\mathrm{M}$ is bounded and has diameter $\leqslant \pi \mathrm{p}$.

Proof. Let $\alpha$ be a geodesic in $M$ from $P$ to $Q$, and assume that $L(\alpha) \geqslant$ $\pi$. Let $C$ be a chain such that $\alpha$ is within $C$, and let $C^{*}$ be the development of C. Then int(im $\alpha) \subseteq$ int $C^{*}$, by Lemma 5.1, since $\alpha$ is too long to fit inside a simplex. Now int $C^{*}$ is smooth: every $X \in$ int $C^{*}$ has a neighbourhood isometric to an open set in an $n$-sphere of radius p. Thus it makes sense to speak of variations of $\alpha$ with fixed endpoints, in the context of smooth differential geometry. A little care is needed at the endpoints, but if I use variations which are sufficiently close to $\alpha$ in the $C^{1}$ topology, they will meet bdy $C^{*}$ only in $\{P, Q\}$. Since $L(\alpha) \geqslant \pi \mathrm{p}$, such variations exist which are shorter than $\alpha$, as is well known from the calculus of variations. It follows that any minimal path from $P$ to $Q$ in $M$ has length $\leqslant \pi$. Hence $M$ is bounded and has diameter $\leqslant \pi p$.

To prove the strict inequality, let $\alpha$ be a path within $C$ from $P$ to $Q$ which is a minimal path of length $\pi \mathrm{p}$, so that $P$ and $Q$ are conjugate. Then $\alpha$ can be varied through geodesics, all having length $\pi \mathrm{p}$. To be precise, say $C=\left(a_{1}, \ldots\right.$, $a_{k}$ ) with $P \in a_{1}$ and $Q \in a_{k}$. Let $\alpha \cap b_{1}=\{X\}$; then $X \in$ int $b_{1}$. Pick $Y \in$ bdy $b_{1}$ and let $\beta$ be the geodesic $X-Y$. For each $u \in \mathrm{I}$, either there is a geodesic $\alpha_{u}$ from $P$ to $Q$ of length $\pi$ p which passes through $\beta(u)$, or else the geodesic from $P$ through $\beta(u)$ meets bdy $C^{*}$ at distance $\leqslant \pi \mathrm{p}$. Let $u^{\prime}$ be the smallest value of $u$ for which the second case occurs (it certainly does occur-when $u=1$, for example). Then $\lim \left\{\alpha_{u}\right.$ as $u \rightarrow u^{\prime}$ from below $\}$ is a path $\alpha_{u^{\prime}}$ in $C^{*}$ from $P$ to $Q$ of length $\pi \mathrm{p}$. On the other hand, int $\alpha_{u^{\prime}} \cap M^{n-2} \neq \varnothing$; so by Lemma $5.1 \alpha_{u^{\prime}}$ is not a geodesic in $\mathbf{M}$ and can therefore be shortened. Thus $\rho_{M}(P, Q) \leqslant \pi$. 
This is a contradiction. Hence $\mathbf{M}$ has diameter $\leqslant \pi \mathrm{p}$, as was to be proved.

Proposition 5.3. Let $\mathrm{M}$ be a $\mathrm{p}$-spherical metric complex ( $\mathrm{p}$ may be infinite) which is an n-manifold without boundary and which satisfies the Curvature Hypothesis for $(n-2)$-simplexes. Then $\mathbf{M}$ satisfies the Curvature Hypothesis.

Proof. I use induction on $n-i$ to prove that if $P \in \operatorname{int} a$ with $\operatorname{dim} a=$ $i \leqslant n-2$, then $\operatorname{dim} \varphi_{P}(\mathbf{M})=i$ and $\kappa_{-}(P \bar{X}) \geqslant 0$ for all $P \bar{X} \subseteq \nu_{P}(M)$.

$T_{P}(\mathrm{M})$ is the orthogonal product of $T_{P}(a)$ with some cone $\mathrm{N}_{P}$. Let $\Sigma_{P}$ be the unit sphere in $\mathbf{N}_{P}$. Using the inductive hypothesis I shall prove that $\Sigma_{P}$ satisfies the hypotheses of Lemma 5.2. It will follow that $N_{P}=\nu_{P}(M)$; hence $\sigma_{P}(M)$ $=\Sigma_{P}$ has diameter $\leqslant \pi$, and the inductive step will be proved.

$\Sigma_{p}$ is a 1-spherical metric complex simplicially isomorphic to $\operatorname{link}(a, \mathbf{M})$. Let $b^{\prime}$ be a $j$-simplex of $\Sigma_{P}$ and let $Q \in$ int $b^{\prime}$. Then $T_{Q}\left(T_{P}(M)\right)$ can be expressed as the orthogonal product $T_{P}(a) \times T_{Q}\left(\mathrm{~N}_{P}\right)=T_{P}(a) \times \mathbf{R}^{1} \times T_{Q}\left(\Sigma_{P}\right)$, where $\mathbf{R}^{1}$ represents $T_{Q}(P \bar{Q})$. Let $\mathbf{T}_{Q}^{*}$ be the orthogonal complement of $Q \bar{P}$ in $T_{Q}\left(T_{P}(\mathrm{M})\right)$. Then $\mathrm{T}_{Q}^{*}$ is isometric to $T_{P}(a) \times T_{Q}\left(\Sigma_{P}\right)=T_{P}(a) \times \varphi_{Q}\left(\Sigma_{P}\right) \times$ $\nu_{Q}\left(\Sigma_{P}\right)$, where $\varphi_{Q}\left(\Sigma_{P}\right)$ is at least $j$-dimensional.

Let $s$ be a small, positive number and let $s_{*}: T_{P}(\mathrm{M}) \rightarrow T_{P}(\mathrm{M})$ be the corresponding dilation (see Lemma 2.5). If $s$ is small enough, then $R=$ $\exp _{P}\left(s_{*}(Q)\right)$ is defined and lies in $\operatorname{int}(a * b)$, where $b \in \operatorname{link}(a, \mathbf{M})$ corresponds to $b^{\prime} \in \Sigma_{P}$. Now $\operatorname{dim}(a * b)=i+j+1$, so by inductive hypothesis, $T_{R}(\mathrm{M})=$ $T_{R}(a * b) \times \nu_{R}(\mathrm{M})$ is the intrinsic factoring of $T_{R}(\mathrm{M})$ into base and normal geometry. Let $\mathrm{T}_{R}^{*}$ be the orthogonal complement of $R \bar{P}$ in $T_{R}(\mathrm{M})$; then $\mathrm{T}_{R}^{*}=$ $\mathbf{R}^{i+j} \times \nu_{R}(\mathrm{M})$. The map $\exp _{P}{ }^{\circ} s_{*}$ induces a map $\mathrm{T}_{Q}^{*} \rightarrow \mathrm{T}_{R}^{*}$ which is anglepreserving; in fact, by the proof of Lemma 2.5, it differs from an isometry only by a dilation. Hence there is an isometry between $T_{P}(a) \times \varphi_{Q}\left(\Sigma_{P}\right) \times \nu_{Q}\left(\Sigma_{P}\right)$ and $\mathrm{R}^{i+j} \times \nu_{R}(\mathrm{M})$. The former has flat part at least $T_{P}(a) \times \varphi_{Q}\left(\Sigma_{P}\right)$, which is at least $(i+j)$-dimensional. It can only be that $T_{P}(a) \times \varphi_{Q}\left(\Sigma_{P}\right)=\mathbf{R}^{i+j}$, so that $\operatorname{dim} \varphi_{Q}\left(\Sigma_{P}\right)=j$, and that $\nu_{Q}\left(\Sigma_{P}\right)=\nu_{R}(\mathbf{M})$. By inductive hypothesis $\sigma_{Q}\left(\Sigma_{P}\right)=$ $\sigma_{R}(M)$ has diameter $\leqslant \pi$. It follows that the Curvature Hypothesis holds at each point $Q \in \Sigma_{p}$. So by Lemma $5.2, \Sigma_{P}$ has diameter $\leqslant \pi$.

The proof of Lemma 5.1 now shows that the base of $\mathbf{N}_{P}$ must be simply $\{P\}$. Hence in the factoring $T_{P}(M)=T_{P}(a) \times N_{P}, T_{P}(a)$ must be the whole flat part of $T_{P}(\mathrm{M})$. Therefore $\varphi_{P}(\mathrm{M})=T_{P}(a)$ has dimension $i$, and $\sigma_{P}(\mathrm{M})=\Sigma_{P}$ has diameter $\leqslant \pi$. The inductive step follows, and Proposition 5.3 is proved.

The first assertion of Theorem 3 is a particular case of Proposition 5.3. To prove the second assertion I shall use spherical approximations to linear metric complexes. Let $\mathbf{M}$ be a linear metric complex. Let $a \in \mathbf{M}$; say $\operatorname{dim} a=k$. Thinking of $a$ as a linear simplex in $\mathbf{R}^{k}$, let the $(k-1)$-sphere circumscribed about $a$ have radius $\mathrm{p}(a)$. If $\mathrm{q}$ is a number $\geqslant\{\mathrm{p}(a)$ for all $a \in \mathrm{M}\}$, then the $\mathrm{q}$ - 
to $\nu_{P^{\prime \prime}}\left(a^{\prime \prime}\right)$ because $b$ is contained in the affine space $\left[C, b^{\prime \prime}\right]$ generated by $C$ and $b^{\prime \prime}$, in which $T_{P^{\prime \prime}}\left(b^{\prime \prime}\right)$ and $C \overline{P^{\prime \prime}}$ are both orthogonal to $\nu_{P^{\prime \prime}}\left(a^{\prime \prime}\right)$. Hence $P \circ \lambda^{*}$ is just orthogonal projection $T_{P}(a) \rightarrow v_{P^{\prime \prime}}\left(a^{\prime \prime}\right)$. As $P$ varies in $b$, so that $P^{\prime \prime}$ varies in $S, v_{P^{\prime \prime}}\left(a^{\prime \prime}\right)$ undergoes parallel translation, since it always remains orthogonal to $\left[C, b^{\prime \prime}\right]$ which remains fixed. (I am abusing notation somewhat by referring to $\nu_{P^{\prime \prime}}\left(a^{\prime \prime}\right)$ when $P^{\prime \prime}$ need not be in $a^{\prime \prime}$; what I really mean is $D \lambda\left(\nu_{P}\left(T_{P^{\prime}}(a)\right)\right)$, where $P$ is the varying point and $P^{\prime}$ its original position.) Thus for the purpose of measuring angles in $v_{P}(a)$ and $v_{P^{\prime \prime}}\left(a^{\prime \prime}\right)$, it does not matter where in $[b]$ I choose $P$. Choose $P$ so that $C \vec{P}$ is orthogonal to $[b]$; then $T_{P^{\prime \prime}}\left(b^{\prime \prime}\right)$ is parallel to $T_{P}(b)$, and $P \circ \lambda^{*}$ reduces to $\lambda^{*}: \nu_{P}(a) \rightarrow \nu_{P^{\prime \prime}}\left(a^{\prime \prime}\right)$. So $f_{a}$ can be calculated using $g \circ \lambda^{*}$.

Let $X, Y \in \sigma_{P}(a)$ and let $\beta$ be the geodesic $X-Y$ in $\sigma_{P}(a)$, so that $\angle \bar{X} P \bar{Y}=$ $L(\beta)$. Set $\beta^{*}=\lambda^{*} \circ \beta, X^{*}=\lambda^{*}(X), Y^{*}=\lambda^{*}(Y)$. Then $\angle \overline{X^{*}} P^{\prime \prime} \overline{Y^{*}}$ is the distance in $\sigma_{P^{\prime \prime}}\left(a^{\prime \prime}\right)$ between $f_{a}(X)$ and $f_{a}(Y)$.

Let $\left(r^{*}, \theta^{*}\right)$ be polar coordinates at $P^{\prime \prime}$ in the plane $\left[P^{\prime \prime}, \beta^{*}\right]$, and let $s$ and $s^{*}$ denote the arc-length functions along $\beta$ and $\beta^{*}$. Then $d s^{*} \geqslant r^{*} d \theta^{*}$; also $d s^{*}$ $\leqslant d s$ since $\lambda^{*}$ is orthogonal projection. Hence $d \theta^{*} \leqslant\left(1 / r^{*}\right) d s$. Now if $\psi(t)$ denotes the angle between $P \overline{\beta(t)}$ and $P^{\prime \prime} \overline{\beta^{*}(t)}$, then $r^{*}(t)=\cos \psi(t)$. But $\psi(t)$ is at most the angle $\psi$ between $[a]$ and $\left[T_{P^{\prime \prime}}\left(a^{\prime \prime}\right)\right]$; and the latter is the tangent plane to $S$ at a point in the polar cap determined by $[a] \cap S$. Hence $\psi \leqslant$ $\sin ^{-1}(\mathrm{p}(a) / \mathrm{q}) \leqslant \sin ^{-1}(\mathrm{p} / \mathrm{q})$. So $r^{*} \geqslant\left[1-(\mathrm{p} / \mathrm{q})^{2}\right]^{1 / 2}$ and $d \theta^{*} \leqslant\left[1-(\mathrm{p} / \mathrm{q})^{2}\right]^{-1 / 2} d s$. It follows that $L \overline{X^{*}} P^{\prime \prime} \overline{Y^{*}} \leqslant\left[1-(\mathrm{p} / \mathrm{q})^{2}\right]^{-1 / 2} L \bar{X} P \bar{Y}$, which is what $\mathrm{I}$ had to prove. The proof of Lemma 5.4 is complete.

LEMMA 5.5. Let $\mathrm{M}$ be a linear metric complex satisfying hypothesis (2) of Theorem 3. Let $\mathrm{q}$ satisfy the inequality of Lemma 5.4 and let $\lambda: \mathbf{M} \rightarrow \mathbf{M}^{\prime \prime}$ be the q-spherical approximation to $\mathbf{M}$. Then for every $P, Q \in \mathbf{M}$,

$$
\rho_{M}(P, Q) \leqslant\left[1-(\mathbf{p} / \mathbf{q})^{2}\right]^{-1 / 2} \rho_{M^{\prime \prime}}(\lambda(P), \lambda(Q)) .
$$

Proof. As said in the proof of Lemma 5.4, I need only prove this result in case $P$ and $Q$ are in the same simplex $a \in \mathbf{M}$. Say $a \subseteq \mathbf{R}^{k}$ and let $\mathbf{S}$ be a sphere with centre $C$ and radius q circumscribing $a$. Let $\alpha$ be the geodesic $P-Q$, and let $s$ be the arc-length function along $\alpha$. Let $(r, \theta)$ be polar coordinates with origin $C$ in the plane $[C, \alpha]$. Similarly let $\alpha^{\prime \prime}$ be the geodesic $\lambda(P)-\lambda(Q)$ in $S$, and let $s^{\prime \prime}$ be the arc-length function along $\alpha^{\prime \prime}$. Then im $\alpha^{\prime \prime}=\lambda(\operatorname{im} \alpha)$, so I can re-paramatrize $\alpha^{\prime \prime}$ as $\lambda \circ \alpha$.

Now $d s^{\prime \prime}=\mathrm{q} d \theta$ and $d s=r d \theta / \sin (L \bar{Q} \alpha(t) \bar{C})$. And $\angle \bar{Q} \alpha(t) \bar{C} \geqslant \cos ^{-1}(\mathrm{p}(a) / \mathrm{q})$ $\geqslant \cos ^{-1}(\mathrm{p} / \mathrm{q})$. Since $r \leqslant q, d s \leqslant\left[1-(\mathrm{p} / \mathrm{q})^{2}\right]^{-1 / 2} d s^{\prime \prime}$. The lemma follows.

Proof of Theorem 3. Combining Lemmas 5.4 and 5.5 gives: whenever $\mathrm{q} \geqslant \mathrm{p}\left[1-((2 \pi-\kappa) / 2 \pi)^{2}\right]^{-1 / 2}$, then $\mathbf{M}$ has diameter $\leqslant \pi \mathrm{q}^{2}\left(\mathrm{q}^{2}-\mathrm{p}^{2}\right)^{-1 / 2}$. The function $F(x)=\pi x /\left(x-\mathrm{p}^{2}\right)^{1 / 2}$ takes on its minimum value $2 \pi \mathrm{p}$ when $x=2 \mathrm{p}^{2}$. 
But $2 \mathbf{p}^{2}$ is a permissible value for $\mathbf{q}$, or the limit of permissible values, only if $\kappa \geqslant\left(2-2^{1 / 2}\right) \pi$. Otherwise $F\left(\mathrm{q}^{2}\right)$ is strictly increasing in $\mathrm{q}^{2}$ and so the best I can infer is diam $M \leqslant \pi \mathrm{p} 4 \pi^{2} /\left[(2 \pi-\kappa)\left(4 \pi \kappa-\kappa^{2}\right)^{3 / 2}\right]$. Thiscompletes the proof of Theorem 3.

THEOREM 4. Let $\mathrm{K}$ be a simplicial 3-manifold without boundary. Assume that every 1-simplex is a face of at most five 3-simplexes. Then $\mathbf{K}$ is finite.

PROOF. Metrize $\mathbf{K}$ by making all the tetrahedra regular, of side length 1 . Then $\mathbf{K}$ is actually a metric complex. The essential ingredient in the proof of this statement is the observation that any finite subcomplex $\mathbf{L}$ of $\mathbf{K}$ can be isometrically embedded as a subcomplex of a regular hyper-simplex with as many vertices as $\mathrm{L}$ has. As was already mentioned in $\S 2$, Example 2, $\mathrm{K}$ satisfies the Curvature Hypothesis for 1-simplexes. The second hypothesis of Theorem 3 is trivial to verify; so Theorem 4 is a particular case of Theorem 3.

THEOREM 2. Let $\mathrm{M}$ be a spherical metric complex which is an n-manifold without boundary, and which satisfies the Curvature Hypothesis for $(n-2)$-simplexes. Then:

(i) M satisfies the Curvature Hypothesis;

(ii) if $n$ is even and $\mathbf{M}$ orientable, then $\mathbf{M}$ is simply-connected;

(iii) if $n$ is odd, then $\mathrm{M}$ is orientable.

Proof. The first assertion is just Proposition 5.3. I shall prove only the second statement; the proof is quite close to that of Synge [14]. The third assertion is proved using the same techniques; see Preissmann [9, Théorème 13"caution: "paire" is a misprint for "impaire"].

Assume for now that $\mathbf{M}$ is $\mathbf{p}$-spherical, with $\mathbf{p}$ finite. Let $\mu \in \pi_{1}(\mathrm{M})$ be $\mathbf{a}$ nonzero free homotopy class (that is, without fixed base-points), and let $\alpha$ be a shortest path representing $\mu$. Then $\alpha$ is a closed geodesic. By Lemma $5.2 \alpha$ has a neighbourhood $\mathbf{U}$ in $\mathbf{M}$ which is a smooth manifold, and $\alpha$ is a smooth curve in U. Let $P=\alpha(0)=\alpha(1)$. Let $\mathbf{N} \subseteq T_{P}(\mathrm{M})$ be the hyperplane orthogonal to $T_{P}(\mathrm{im} \alpha)$. Then parallel translation around $\alpha$ induces a linear isometry $L: \mathrm{N} \rightarrow$ $\mathbf{N}$. The hypotheses on $\mathbf{M}$ ensure that $L$ keeps some nonzero vector fixed; this vector determines a parallel vector-field $V$ around $\alpha$. Since $\mathrm{U}$ has constant positive sectional curvature everywhere, it is clear that varying $\alpha$ in the direction of $V$ reduces its length. This is a contradiction; hence $\pi_{1}(M)=0$.

If $M$ is a linear metric complex, the same argument can be applied. This time $\mathrm{U}$ is flat, so translating $\alpha$ in the direction of $V$ preserves $L(\alpha)$. However, as in the proof of Lemma 5.2, I can translate $\alpha$ until it meets the $(n-2)$-skeleton of $\mathbf{M}$, and then $\alpha$ can be shortened. This is again a contradiction; and the Theorem is proved. 
spherical approximation $\lambda: M \rightarrow M^{\prime \prime}$ is defined; see $\S 4$. The second hypothesis of Theorem 3 implies that $\mathrm{p}(a) \leqslant \mathrm{p}$ for every $a \in \mathbf{M}$. Hence the q-spherical approximation to $M$ exists for every $q \geqslant p$.

LEMMA 5.4. Let $\mathrm{M}$ satisfy the hypotheses of Theorem 3. Let $\mathrm{q} \geqslant \mathrm{p}[1-$ $\left.((2 \pi-\kappa) / 2 \pi)^{2}\right]^{-1 / 2}$ and let $\mathbf{M}^{\prime \prime}$ be the q-spherical approximation to $\mathrm{M}$. Then $\mathbf{M}^{\prime \prime}$ satisfies the Curvature Hypothesis.

Proof. I know that for each $P \in \mathbf{M}^{n-2}, \sigma_{P}(\mathbf{M})$ has diameter $\leqslant(2 \pi-\kappa) / 2$; it suffices to prove that $\sigma_{\lambda(P)}\left(M^{\prime \prime}\right)$ has diameter $\leqslant\left[1-(\mathrm{p} / \mathrm{q})^{2}\right]^{-1 / 2}(2 \pi-\kappa) / 2$; for then the condition on $q$ ensures that this is $\leqslant \pi$.

The proofs of this lemma and the next are based on this simple observation: Let $f: \mathbf{M} \rightarrow \mathbf{M}^{\prime \prime}$ be a homeomorphism between spherical metric complexes such that:

$f$ is a simplicial isomorphism;

if $P-Q$ is a geodesic segment in a simplex of $\mathbf{M}$, then im $f(P-Q)$ is the geodesic segment $f(P)-f(Q)$ in $\mathbf{M}^{\prime \prime}$;

there is a number $N$ such that if $P, Q \in a$, a simplex of $\mathbf{M}$, then $\rho_{M}{ }^{\prime \prime}(f(P)$, $f(Q)) \leqslant N \rho_{M}(P, Q)$.

Then this inequality holds for any two points of $\mathbf{M}$.

For let $\alpha$ be a minimal path from $P$ to $Q$ in $\mathbf{M}$, and let $C$ be a chain within which $\alpha$ lies. Then $\operatorname{im} f(\alpha)$ is a path from $f(P)$ to $f(Q)$ within $f\left(C^{\prime}\right)$, and $\operatorname{im} f(\alpha)$ has length $\leqslant N L(\alpha)$. The desired inequality follows.

In the present case I take $N=\left[1-(\mathrm{p} / \mathrm{q})^{2}\right]^{-1 / 2}$. I must now define the map $f: \sigma_{P}(\mathrm{M}) \rightarrow \sigma_{\lambda(P)}\left(\mathrm{M}^{\prime \prime}\right)$. Say $P \in$ int $b$, and let $a$ have $b$ as face. Think of $a$ as a $k$-simplex in $\mathbf{R}^{k}$. Let $\mathbf{S}$ be a sphere with centre $C$ and radius q circumscribed about $a$. Then radial projection of $a$ from $C$ into $S$ defines $\lambda: a \rightarrow a^{\prime \prime}$. Set $P^{\prime \prime}=\lambda(P), b^{\prime \prime}=\lambda(b)$.

$\lambda$ induces a linear map $D \lambda: T_{P}(a) \rightarrow T_{P^{\prime \prime}}\left(a^{\prime \prime}\right)$, and $D \lambda\left(T_{P}(b)\right)=T_{P^{\prime \prime}}\left(b^{\prime \prime}\right)$. Now $\nu_{P}(a)$ is the section of $T_{P}(a)$ by the affine space through $P$ orthogonal to $b$. Hence $D \lambda\left(\nu_{P}(a)\right)$ is the section of $T_{P^{\prime \prime}}\left(a^{\prime \prime}\right)$ by an affine space through $P^{\prime \prime}$ which meets $T_{P^{\prime \prime}}\left(b^{\prime \prime}\right)$ only in $P^{\prime \prime}$. Let $P: T_{P^{\prime \prime}}\left(a^{\prime \prime}\right) \rightarrow \nu_{P^{\prime \prime}}\left(a^{\prime \prime}\right)$ be the orthogonal projection. Then $P \circ D \lambda: v_{P}(a) \rightarrow \nu_{P^{\prime \prime}}\left(a^{\prime \prime}\right)$ is a linear isomorphism. Let $g$ : $\left(\nu_{P^{\prime \prime}}\left(a^{\prime \prime}\right)-\left\{P^{\prime \prime}\right\}\right) \rightarrow \sigma_{P^{\prime \prime}}\left(a^{\prime \prime}\right)$ be radial projection from $P^{\prime \prime}$, and set $f_{a}=g \circ P \circ$ $D \lambda: \sigma_{P}(a) \rightarrow \sigma_{P^{\prime \prime}}\left(a^{\prime \prime}\right)$. If $b<c<a$, then $f_{c}$ is, up to a natural isometry, just the restriction of $f_{a}$. Hence $f=\bigcup\left\{f_{a}\right.$ for $\left.b<a\right\}: \sigma_{P}(\mathbf{M}) \rightarrow \sigma_{P^{\prime \prime}}\left(\mathbf{M}^{\prime \prime}\right)$ is well defined.

I now give a different construction of $f_{a}$, which seems to me easier to calculate with, but is not so clearly intrinsic. Let $\lambda^{*}: T_{P}(a) \rightarrow T_{P^{\prime \prime}}\left(a^{\prime \prime}\right)$ be parallel projection in the direction $C \bar{P}$. Then $\lambda^{*}(P)=P^{\prime \prime}$ and for each ray $P \bar{X} \subseteq T_{P}(a)$, the images $\lambda^{*}(P \bar{X})$ and $D \lambda(P \bar{X})$ are equal. The affine space $T_{P}(b)$ is orthogonal 


\section{REFERENCES}

1. A. D. Aleksandrov and V. A. Zalgaller, Two-dimensional manifolds of bounded curvature, Trudy Mat. Inst. Steklov. 63 (1962); English transl., The intrinsic geometry of surfaces, Transl. Math. Monographs, vol. 15, Amer. Math. Soc., Providence, R. I., 1967. MR 27 \#1191.

2. T. F. Banchoff, Critical points and curvature for embedded polyhedra, J. Differential Geometry 1 (1967), 245-256. MR 37 \#921.

3. É. Cartan, Lecons sur la géométrie des espaces de Riemann, 2ième éd., GauthierVillars, Paris, 1926, 1946. MR 8, 602.

4. H. R. Gluck, Piecewise linear methods in Riemannian geometry, Univ. of Pennsylvania, 1972 (mimeographed notes).

5. J. Hadamard, Les surfaces à courbures opposées, J. Math. Pures Appl. (5) 4 (1898), $27-73$.

6. J. Milnor, Morse theory, Ann. of Math. Studies, no. 51, Princeton Univ. Press, Princeton, N. J., 1963. MR 29 \#634.

7. S. B. Myers, Riemannian manifolds with positive mean curvature, Duke Math. J. 8 (1941), 401-404. MR 3, 18.

8. H. A. Osborn, Function algebras and the deRham theorem in PL, Bull. Amer. Math. Soc. 77 (1971), 386-391. MR 43 \#2717.

9. A. Preissmann, Quelques propriétés globales des espaces de Riemann, Comment. Math. Helv. 15 (1943), 175-216. MR 6, 20.

10. W. Rinow, Die innere Geometrie der metrischen Räume, Die Grundlehren der math. Wissenschaften, Band 105, Springer-Verlag, Berlin, 1961. MR 23 \#A1290.

11. C. P. Rourke and B. J. Sanderson, Introduction to piecewise-linear topology, Springer-Verlag, Berlin, 1972.

12. J. R. Stallings, The piecewise-linear structure of Euclidean space, Proc. Cambridge Philos. Soc. 58 (1962), 481-488. MR 26 \#6945.

13. D. A. Stone, Sectional curvature in piecewise linear manifolds, Bull. Amer. Math. Soc. 79 (1973), 1060-1063. MR 47 \#9507.

14. J. L. Synge, On the connectivity of spaces of positive curvature, Quart. J. Math. 7 (1936), 316-320.

DEPARTMENT OF MATHEMATICS, STATE UNIVERSITY OF NEW YORK, STONY BROOK, NEW YORK 11794

Current address: Department of Mathematics, Brooklyn College of the City University of New York, Brooklyn, New York 11210 